\title{
A HARDWARE ARCHITECTURE FOR GPS/INS-ENABLED WIRELESS SENSOR NETWORKS
}

by

CHUN TANG

A thesis submitted to the Department of Electrical and Computer Engineering

in conformity with the requirements for

the degree of Master of Applied Science

Queen's University

Kingston, Ontario, Canada

(December, 2011)

Copyright CChun Tang, 2011 


\begin{abstract}
Wireless sensor network technology has now been widely adopted. In many applications, distributed sensor nodes collect data at different locations and the location information of each node is required. The Global Positioning System is commonly used to identify the location of the nodes in such networks. Although GPS localization has consistent long-term accuracy, it is limited by the inherent dependency on a direct line of sight to 4 or more external satellites. The increasing demand for an embedded system providing reliable navigation solutions regardless of its operational environment has motivated investigations into the use of integrated systems that combine inertial sensors with GPS receivers.

This research proposes a hardware architecture for location-based wireless sensor networks. In this architecture, each sensor node consists of a GPS receiver, a reduced set of low cost micro-electromechanical-system-based INS and a wireless transceiver. Sensor nodes in WSN are often equipped with irreplaceable batteries, which makes the power consumption crucial. To reduce the energy consumption, a microcontroller is used to control the power supply. Besides, a motion detection scheme is proposed by taking advantage of the ultra low-power wake-up function of the microcontroller. A low-power featured digital signal processor is used to accomplish the navigation computation using the Kalman filter for GPS/INS data fusion. Non-Holonomic Constraints derived velocity updates are applied to reduce the position errors. Field tests are conducted to verify the realtime performance of the proposed system with a positioning update rate of $20 \mathrm{~Hz}$.

The first test shows that the 2D INS/GPS integration can maintain the average system position error within 5 meters during a 60-second GPS outage. The second test used low cost inertial sensors. The average position error was 10.17 meters during a 20 -second outage. The largest RMS value of position errors among these outages was within 14.5 meters. Furthermore, additional accuracy improvements of approximately 1.4 meters were achieved by utilizing NHC during GPS outages. The
\end{abstract}


third test shows that the average error during a 30 -second outage is approximately 20.6 meters for the on-foot scenario and 26.7 meters for the in-vehicle scenario. 


\section{Acknowledgements}

Firstly, I would like to express my deepest gratitude to my supervisor, Dr. Mohamed Ibnkahla, for his guidance, support and encouragement. Dr. Ibnkahla's knowledge and insight were invaluable resources to me throughout my graduate studies. I would also like to thank Dr. Aboelmagd Noureldin for his unconditional support and invaluable advice. Also, a sincere thank you to Zhi Shen, Walid Farid Abd El Fatah and Tashfeen Karamat for their patient explanations, discussion, and for familiarizing me with the navigation devices in their laboratory. I would especially like to acknowledge Walid for providing the dataset for the first field test in this thesis work.

I would like to extend my thanks to the members of Wireless Sensor Network and Communication Laboratory: Basel Nabulsi, Abdallah AlMaaitah, Amr El Mougy, Peng Hu, Gayathri Vijay, Ala Abu Alkheir, Zouheir El-Jabi and Xiao Zhao. They always provided me with the help and suggestions I needed. Their support made it possible for me to complete this work.

Finally and most importantly, I would like to thank my parents for their unconditional love and care. This work would not have been possible without their support. 


\section{Table of Contents}

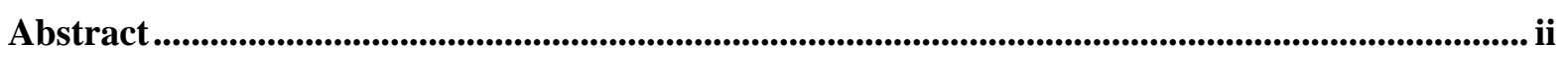

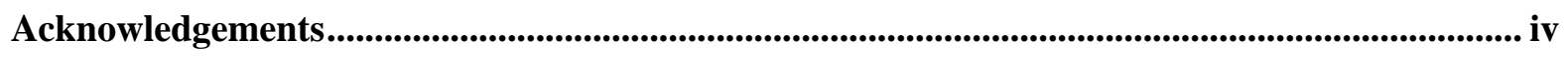

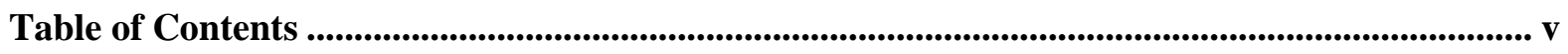

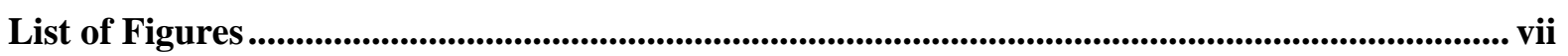

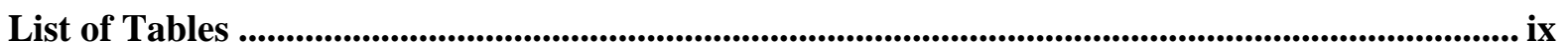

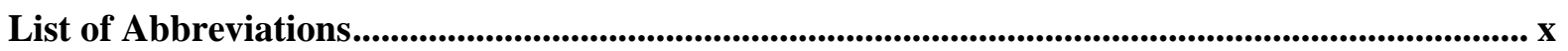

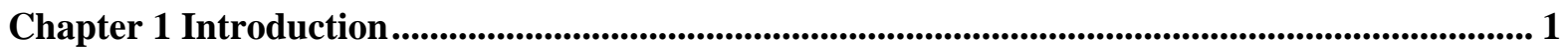

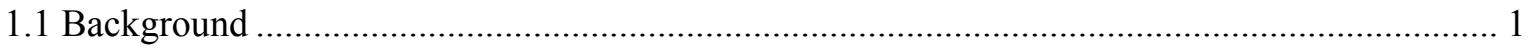

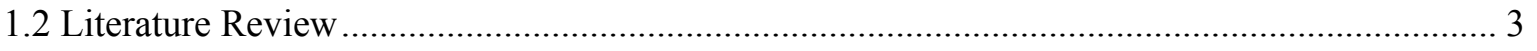

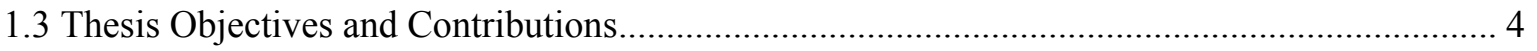

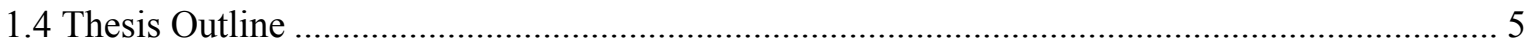

Chapter 2 WSN and Navigation System Overview....................................................................... 7

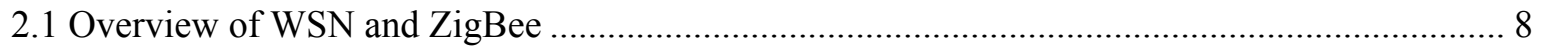

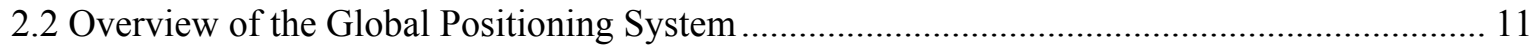

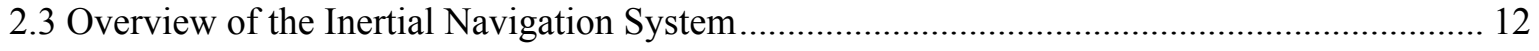

2.3.1 Mechanization and Quaternion Transformation ............................................................. 13

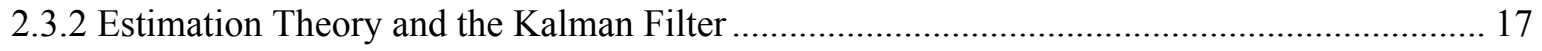

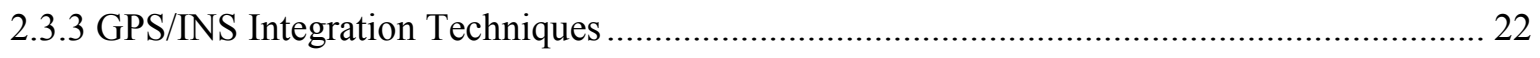

Chapter 3 Accuracy Improvement Methods for Low Cost Systems ................................................. 26

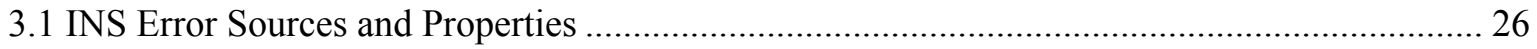

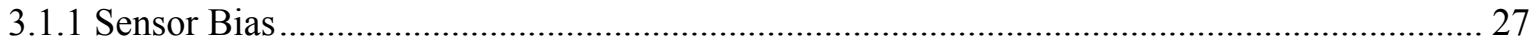

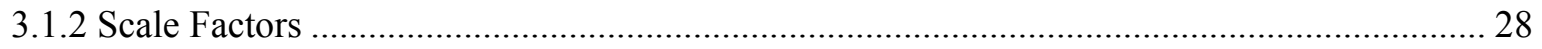

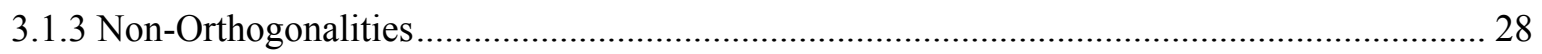

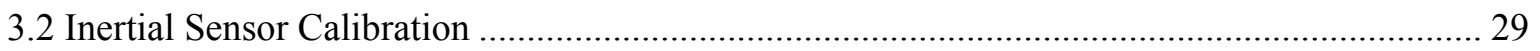

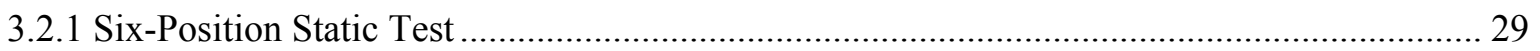

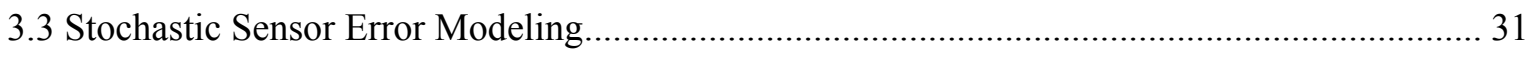

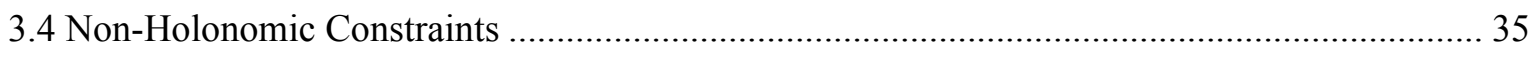

Chapter 4 Hardware and Software Implementation .............................................................................. 38 


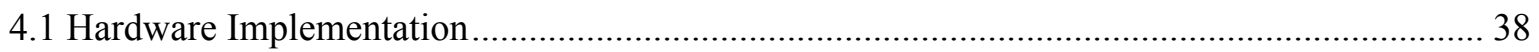

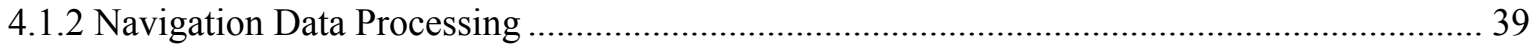

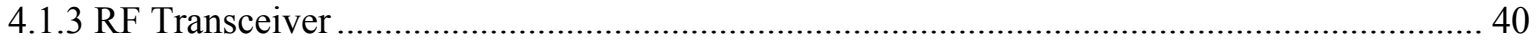

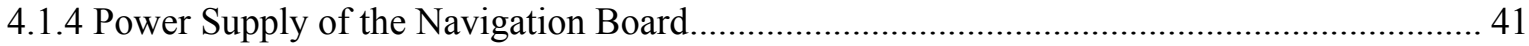

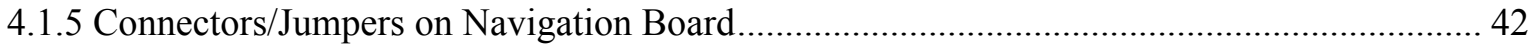

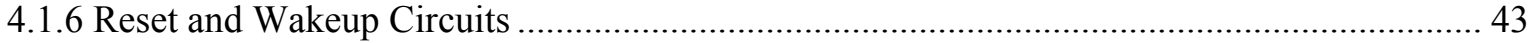

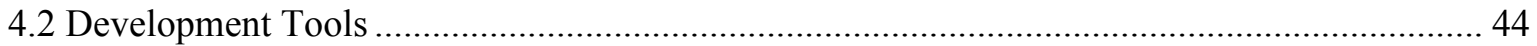

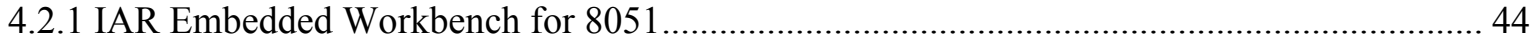

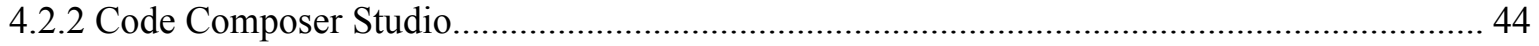

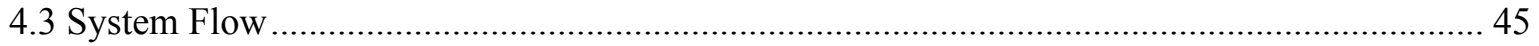

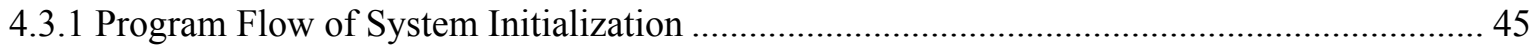

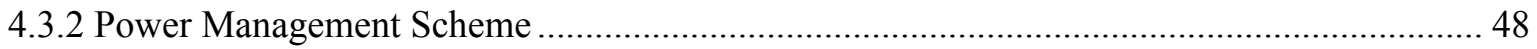

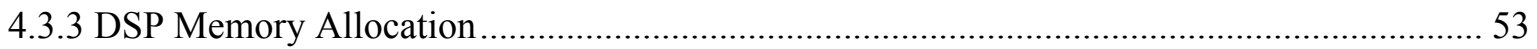

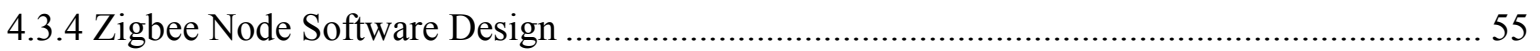

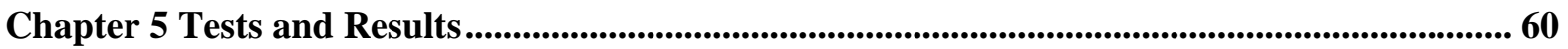

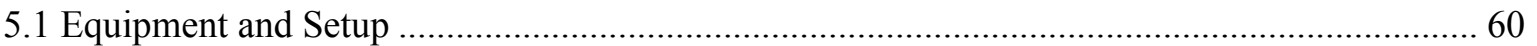

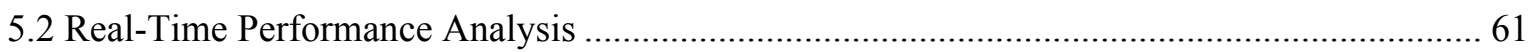

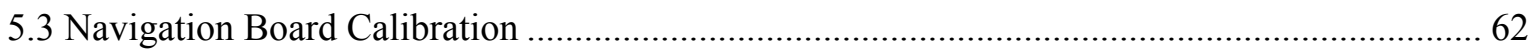

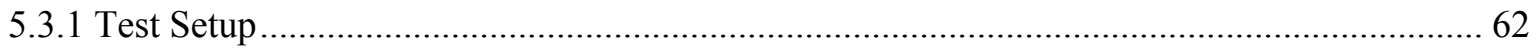

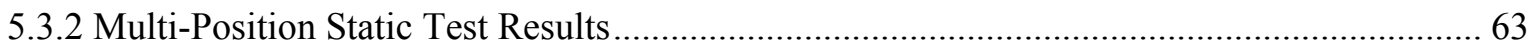

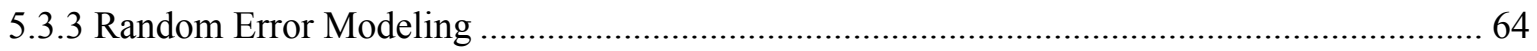

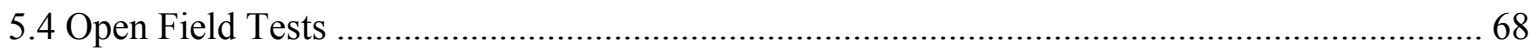

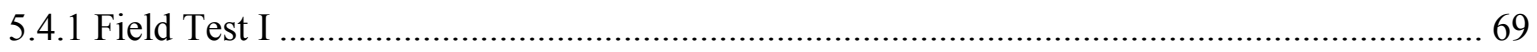

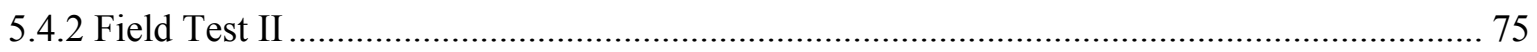

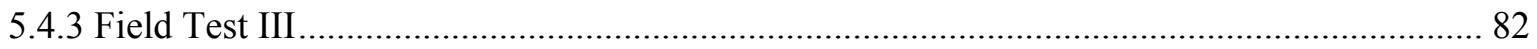

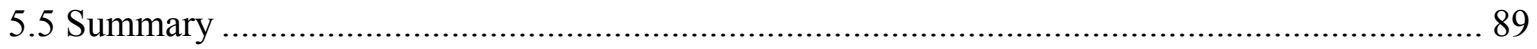

Chapter 6 Summary, Conclusions and Recommendations ............................................................. 91

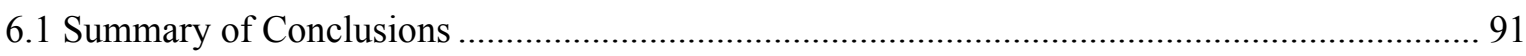

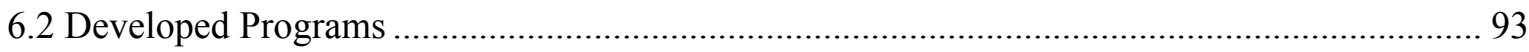

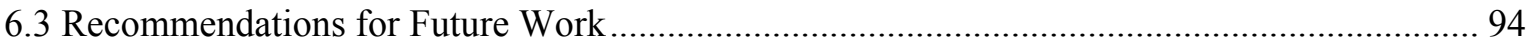

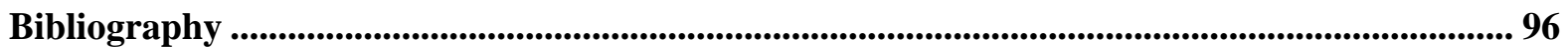

Appendix A Schematics of the Navigation Board ................................................................................... 99 


\section{List of Figures}

Figure 2.1 The architecture of the proposed system. ................................................................... 7

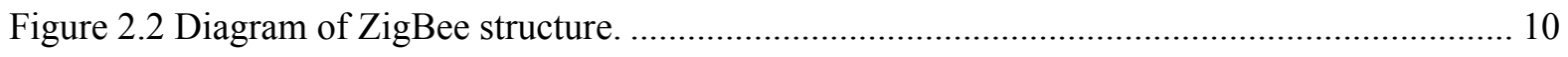

Figure 2.3 The architecture of a typical wireless sensor network .................................................. 10

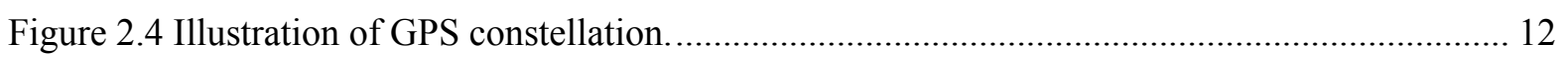

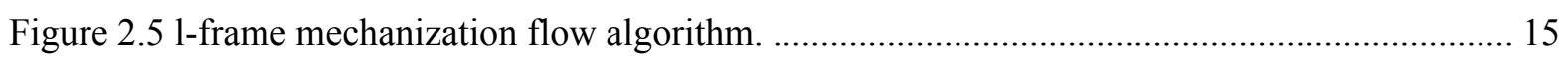

Figure 2.6 A complete picture of the two-step Kalman filter operations......................................... 21

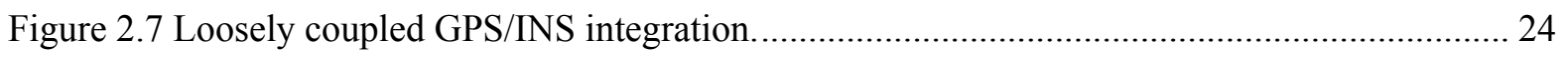

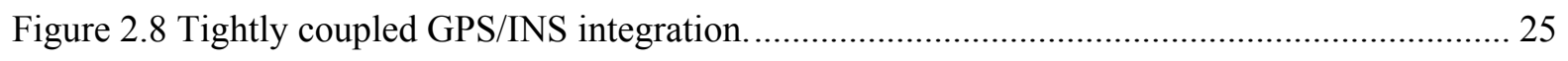

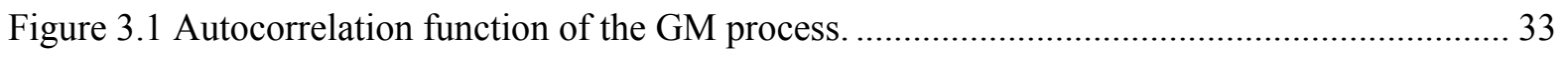

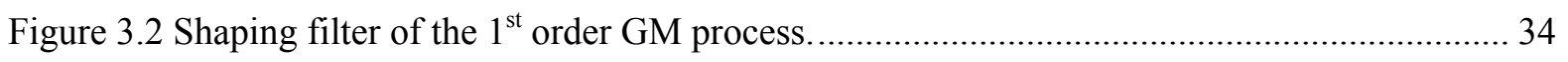

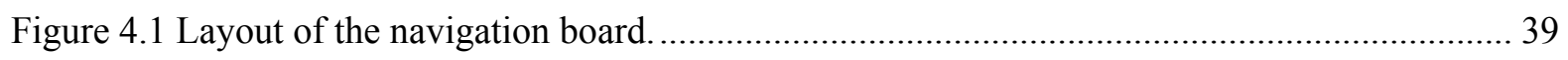

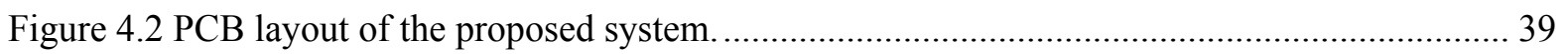

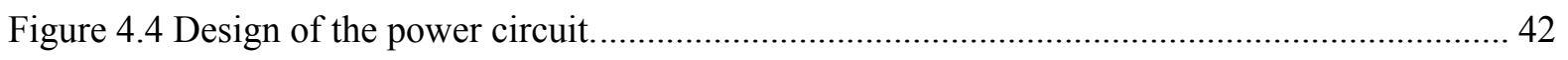

Figure 4.5 Power control design with a 2-pin jumper and a bipolar junction transistor.................... 43

Figure 4.6 DSP initialization routine and DSP reset ISR ............................................................ 47

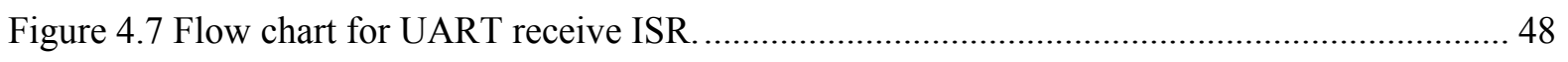

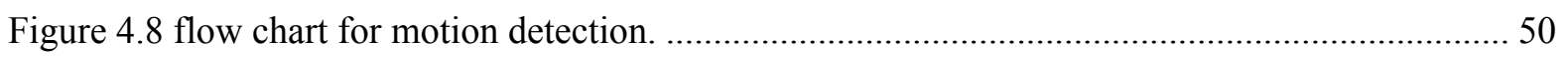

Figure 4.9 Program flows of DSP_Sleep and Wakeup_ISR ...................................................... 52

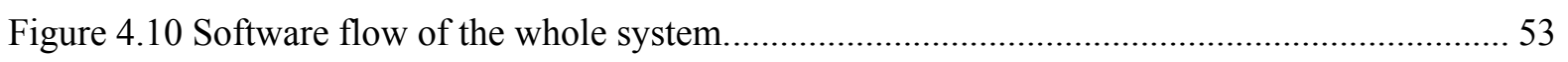

Figure 4.11 The structure of a typical ZigBee sensor network ...................................................... 56

Figure 4.12 The initialization and configuration of the sink node program flow. ............................ 57

Figure 4.13 The event processing flow of the sink node task event handler. …................................ 58

Figure 4.14 The event processing flow of the sensor node task event handler.................................59

Figure 5.1 A typical time frame within one second of DSP computation......................................... 62

Figure 5.2 The autocorrelation of $\mathrm{x}$-axis accelerometer reading of 2-hour stationary dataset after

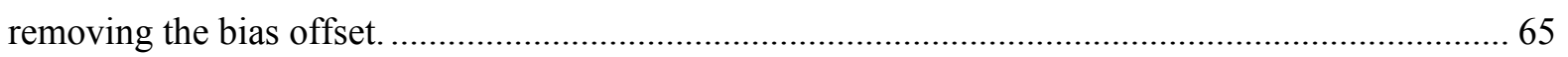

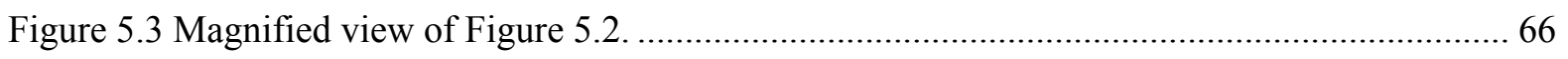

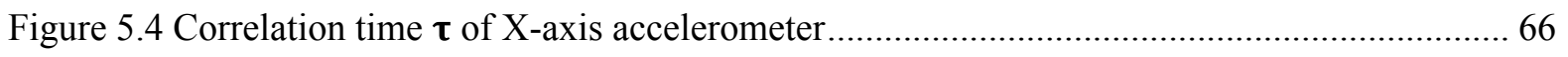

Figure 5.5 The autocorrelation of $y$-axis accelerometer reading of 2-hour stationary dataset after

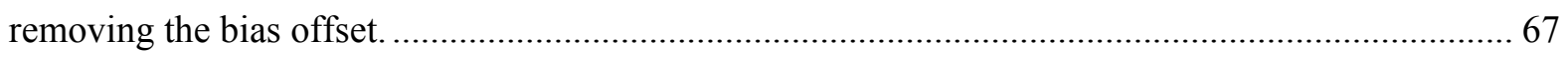

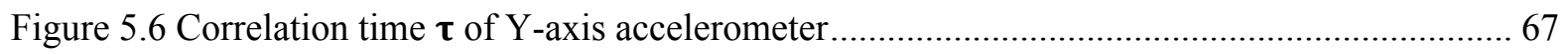


Figure 5.7 The autocorrelation of z-axis gyroscope reading of 2-hour stationary dataset after

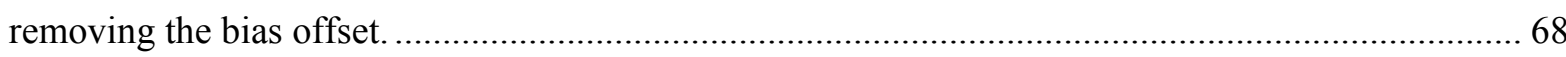

Figure 5.8 Field test I: Reference trajectory and KF trajectory with 4 simulated GPS outages. ......... 72

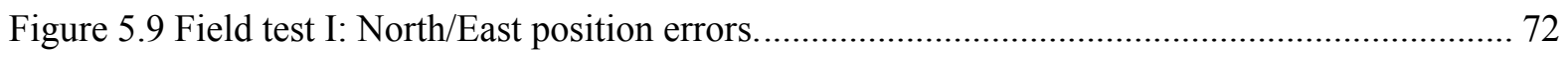

Figure 5.10 Field test I: Reference north velocity and KF north velocity....................................... 73

Figure 5.11 Field test I: Reference east velocity and KF east velocity.......................................... 73

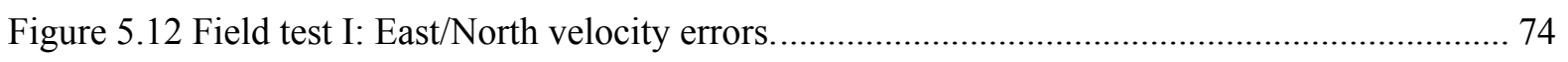

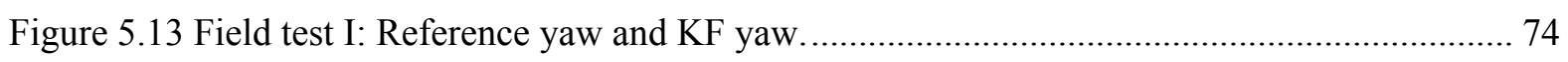

Figure 5.14 Field test II: GPS trajectory and KF trajectories with 4 simulated GPS outages. ............ 77

Figure 5.15 Field test II: North position errors. ....................................................................... 77

Figure 5.16 Field test II: East position errors........................................................................... 78

Figure 5.17 Field test II: GPS north velocity and KF north velocity.............................................. 78

Figure 5.18 Field test II: GPS east velocity and KF east velocity. .............................................. 79

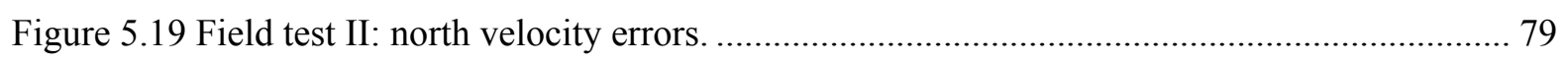

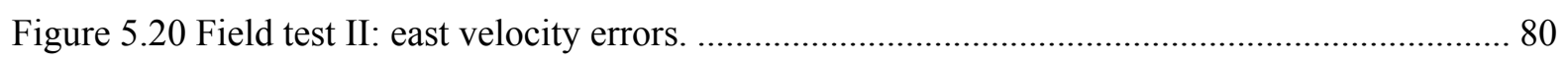

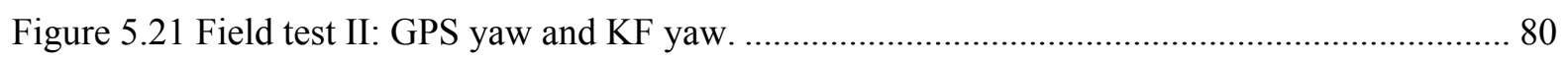

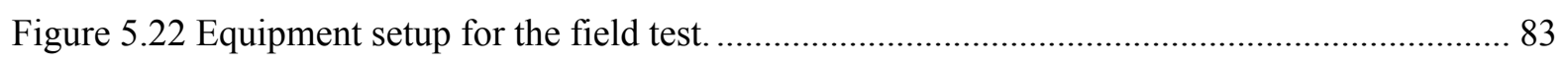

Figure 5.23 Test III.1: GPS trajectory and on-foot Trajectory with 6 GPS outages......................... 85

Figure 5.24 Test III.2: GPS trajectory and on-foot trajectory with 5 GPS outages. ......................... 85

Figure 5.25 Test III.3: GPS trajectory and on-foot trajectory with 5 GPS outages. ........................... 86

Figure 5.26 Test III.4: GPS trajectory and in-vehicle trajectory with 5 GPS outages....................... 86

Figure 5.27 Test III.5: GPS trajectory and in-vehicle trajectory with 4 GPS outages. ...................... 87

Figure 5.28 Test III.6: GPS trajectory and in-vehicle trajectory with 5 GPS outages....................... 87 


\section{List of Tables}

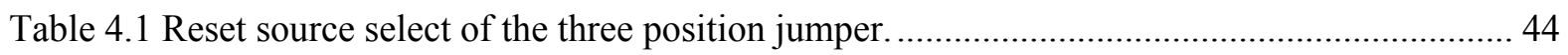

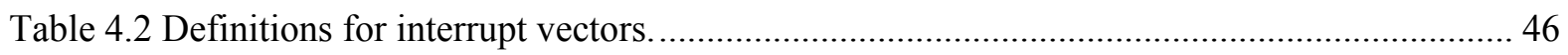

Table 4.3 DSP computation accuracy compared with matlab........................................................... 54

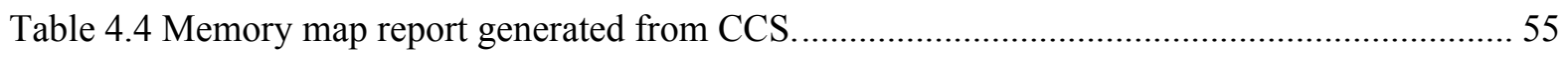

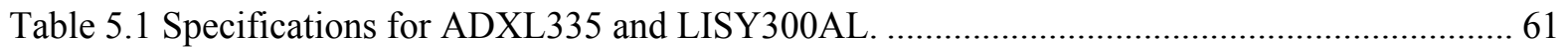

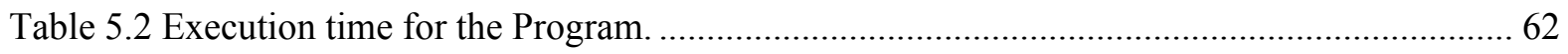

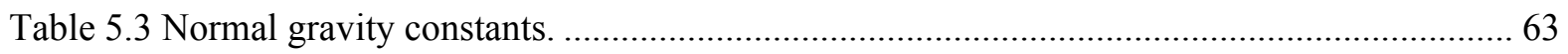

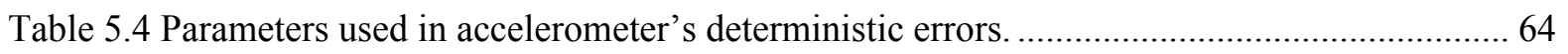

Table 5.5 First order GM model parameters used in field test II. ..................................................... 65

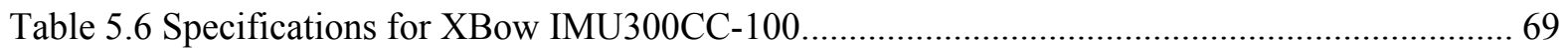

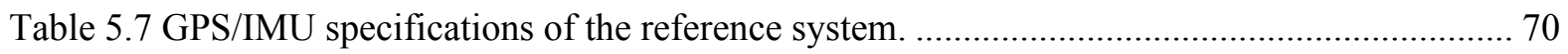

Table 5.8 Field test I: Position/Velocity errors during GPS outages. ................................................ 75

Table 5.9 Field test II: Position/Velocity errors during GPS outages........................................... 81

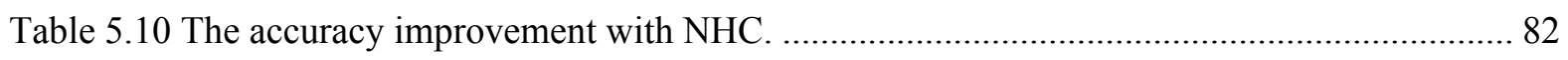

Table 5.11 Specifications for ADIS16003, ADIS16060 and HMC6352 …...................................... 84

Table 5.12 On-foot field test: Position errors during GPS outages.................................................. 88

Table 5.13 In-vehicle field test: Position errors during GPS outages. ........................................... 89 


\section{List of Abbreviations}

2D

3D

ADC

API

AR

ASCII

BJT

C/A

CCS

CEP

COFF

CPLD

DARAM

DCM

DMA

DR

DSP

ECEF

ENU

FPGA

GM

GPIO

GPS

HAL

I2C

IAE
Two-Dimensional

Three-Dimensional

Analog-to-Digital Converter

Application Programming Interface

Auto-Regressive

American Standard Code for Information Interchange

Bipolar Junction Transistor

Coarse-Acquisition

Code Composer Studio

Circular Error Probable

Common Object File Format

Complex Programmable Logic Device

Dual Access Random Access Memory

Direction Cosine Matrix

Direct Memory Access

Dead Reckoning

Digital Signal Processor

Earth Centered Earth Fixed Frame

East-North-Up

Field-Programmable Gate Array

Gaussian-Markov

General Purpose Input/Output

Global Positioning System

Hardware Abstraction Layer

Inter-Integrated Circuit

Innovation-based Adaptive Estimation 


\begin{tabular}{|c|c|}
\hline IDE & Integrated Development Environment \\
\hline IEEE & Institute of Electrical and Electronics Engineers \\
\hline IMU & Inertial Measurement Unit \\
\hline ISR & Interrupt Service Routine \\
\hline JTAG & Joint Test Action Group \\
\hline KF & Kalman Filter \\
\hline MAC & Media Access Control \\
\hline MCU & Micro-Controller Unit \\
\hline MEMS & Micro-Electro-Mechanical Systems \\
\hline MMAE & Multiple-model-based Adaptive Estimation \\
\hline NED & North-East-Down \\
\hline NHC & Non-Holonomic Constraints \\
\hline NMEA & National Marine Electronics Association \\
\hline OSAL & Operating System Abstraction Layer \\
\hline PAN & Personal Area Network \\
\hline PER & Packet Error Rate \\
\hline PLL & Phase-Locked Loop \\
\hline PPM & Parts Per Million \\
\hline PRN & Pseudo-Random Noise \\
\hline PSD & Power Spectral Density \\
\hline RF & Radio Frequency \\
\hline RSSI & Received Signal Strength Indicator \\
\hline SARAM & Single Access Random Access Memory \\
\hline SFR & Special Function Register \\
\hline SINS & Strap-down Inertial Navigation System \\
\hline SPI & Serial Peripheral Interface \\
\hline SSM & State Space Model \\
\hline
\end{tabular}


UART

UKF

ZDO
Universal Asynchronous Receiver/Transmitter

Unscented Kalman Filter

Zigbee Device Object 


\section{Chapter 1}

\section{Introduction}

\subsection{Background}

The location information is usually important in wireless sensor networks (WSN). It is not only required by specific WSN applications such as environment monitoring applications, but also used to improve the routing of WSN, in particular by forwarding the control messages only to a portion of the network and avoid to waste energy on the nodes that do not contain the route to the destination node. A typical example is the Location-Aided Routing (LAR) protocol that utilizes location information to decrease overhead of route discovery [1]. Many other routing protocols such as GeRAF, GeoAODV and LACBER etc. are also based on the geographical location of the nodes [2-4].

The Global Positioning System (GPS) is usually used to identify the spatial coordinates of a sensor node in a WSN. Generally, GPS requires direct Line-Of-Sight (LOS) signals from at least four satellites to figure out where the receiver is. Therefore, a stand-alone GPS may often suffer from signal blockages in degraded signal environments, such as indoor environments, urban canyons, foliated areas, etc. The integration of a Strap-down Inertial Navigation System (SINS) with the Global Positioning System (GPS) has been extensively studied and deployed in different applications. In these integrated systems, SINS provides position, velocity and orientation information at very high data rates (usually above $50 \mathrm{~Hz}$ ) and provides accurate outputs in a short term. However, its performance deteriorates with time quickly. Therefore, GPS and SINS are complementary. Each 
system compensates for the other's drawbacks and it is an efficient way to integrate GPS with SINS for continuously getting reliable navigation information.

Standard Inertial Navigation Systems use high cost accelerometers and gyroscopes to provide precise navigation information, which lessens their popularity in general user-end devices. With the advancement of Micro Electro Mechanical System (MEMS) technology, low-cost MEMS inertial sensors provide a more affordable solution for GPS/INS integrated navigation systems [5]-[6]. Although MEMS sensors make the navigation system less expensive, more compact and more power efficient, the performance is relatively poor due to its high instrument bias and drift, its vulnerability to temperature effects, etc. As a result, the INS errors accumulate rapidly in just a short time interval unless there are updates from external navigation measurements. These errors can be usually reduced through robust integration algorithms with regular updates that ensure an acceptable level of accuracy. A typical example of this is the Kalman filter.

The Kalman Filter (KF), which is named after Rudolf E. Kalman, is commonly used to perform the GPS/INS data fusion in various applications [7-9]. However, the Kalman Filter has some drawbacks. Since it is the optimal filter for modeled processes, predefined system dynamic models are required. If the input data does not fit those models, the positioning accuracy will be significantly degraded [10]; another major problem with KF is the observability of some of its error states; moreover, the performance of the Kalman Filter is poor if the sensor noise level is high [11]. Although some alternative algorithms for INS/GPS integration have been investigated and proved efficient in navigation applications (e.g. Artificial Neural Networks [12]), KF is still computationally 
efficient, and particularly suitable for real-time applications. Therefore, we use KF as the navigation algorithm to fuse the data outputs from the GPS and inertial sensors.

\subsection{Literature Review}

Recently, research efforts have been focused on GPS/INS integration techniques implemented on real-time embedded systems in which the navigation computation is strictly limited by time without any post-processing aid. In real-time applications, inertial sensors based on MEMS technology play an essential part to make the whole system cost-effective and compact.

A Micro-miniature Inertial Measurement Unit (MIMU) and GPS-based integration system has been proposed by [13]. This system is based on a PC/104 embedded microcomputer and the MIMU module is composed of three MEMS gyroscopes and three MEMS accelerometers. Six inertial sensor signals are processed by a multiplexer-based ADC on a customized data acquisition board. The Micro-programming Controlled Direct Memory Access (MCDMA) technique is introduced to improve real-time data calculation and achieve fast data exchange.

Qu et al. [14], have described a miniaturized/low-cost navigation system in which a Digital Signal Processor (DSP) and a Complex Programmable Logic Device (CPLD) are used as a math coprocessor for developing the hardware system. In this proposed system design, CPLD, which has a fast calculation speed, is mainly used for matrix calculation, whereas DSP is used to control and schedule the entire system.

V. Agarwal, H. Arya and S. Bhaktavatsala [15], in their paper describe development of a compact and low-power GPS/INS system based on a FPGA and a floating point DSP. In this paper, 
they used the FPGA to construct an efficient interface for GPS data acquisition. An internal Dual Port Random Access Memory (DPRAM) within FPGA was used for asynchronous data transmission between the GPS and DSP, which significantly reduced the GPS processing overhead on the navigation processor. It adopted a 16 bit, $250 \mathrm{kHz}$ ADC (ADS8364, Texas Instruments [16]) for sampling all the analog signals from inertial sensors simultaneously, rather than using an ADC multiplexer to sample the channels at different time instants.

\subsection{Thesis Objectives and Contributions}

The primary objective of this thesis is to propose an efficient (compact, low-cost, low-power and light weight) DSP-based real-time navigation system design for sensor nodes in WSN where cost, size and power are focal concerns.

During the course of this work, the following tasks were investigated and carried out:

- Propose a novel architecture for WSN by using GPS/INS integrated system that enables efficient navigation/localization under signal degraded environment.

- Implement the proposed system design in hardware and optimize it for low-cost, low-power and compact requirements.

- Develop an external-interrupt triggered power management scheme based on the ultra-low-power wake-up function of a microcontroller to minimize the system power consumption.

- Verify the system performance through open area field tests with simulated as well as actual GPS outages for evaluation of prediction accuracy. 


\subsection{Thesis Outline}

The remainder of this thesis is organized as follows:

Chapter 2 reviews the characteristics of GPS and INS separately, followed by the GPS/INS integration techniques. The basic concepts of GPS will first be provided. A review of INS is described afterwards with a brief introduction of coordinate frame and relevant coordinate transformation, as well as the mechanization equations that are usually used in inertial data processing. The Kalman Filter algorithm is also presented.

Chapter 3 discusses various inertial sensor error sources and their properties. A deterministic error model is derived in matrix form and a standard calibration method is introduced. Next, stochastic error modeling is discussed and the process model state equation of EKF for the first order Gauss-Markov (GM) process is derived in matrix form. Additionally, non-holonomic constraints are also discussed.

Chapter 4 gives an overview of the total integrated system architecture and describes both the hardware and software implementation details of the navigation board. After a brief system hardware description, software development tools are introduced followed by the system program flow with emphasis given to the system power management scheme.

In Chapter 5, static calibration and random error modeling in the laboratory are carried out and the results are analyzed. Also, the real-time performance analysis using CCS simulator is presented. Results of field testing in open areas are presented in this chapter as well. The main idea aims to evaluate the system performance during GPS signal outages. Non-holonomic constraints are applied in the second field test to compare the positional errors during GPS outages. The experimental work 
also includes the verification of the proposed navigation board for both the on-foot and in-vehicle scenarios.

Chapter 6 summarizes the contributions of this thesis and provides suggestions for future research. 


\section{Chapter 2}

\section{WSN and Navigation System Overview}

A new architecture for GPS/INS-enabled WSNs is proposed in this thesis. The sensor node of this system is featured with miniaturized dimension, improved power efficiency and processing overheads. There are mainly 5 blocks in the proposed system (Figure 2.1), which will be presented in details in Chapter 4:

- Data Acquisition Components: accelerometer, gyroscope, GPS, magnetic sensor

- Data Processing Unit: DSP

- Power Management Unit: microcontroller

- Wireless transceiver

- Power supply

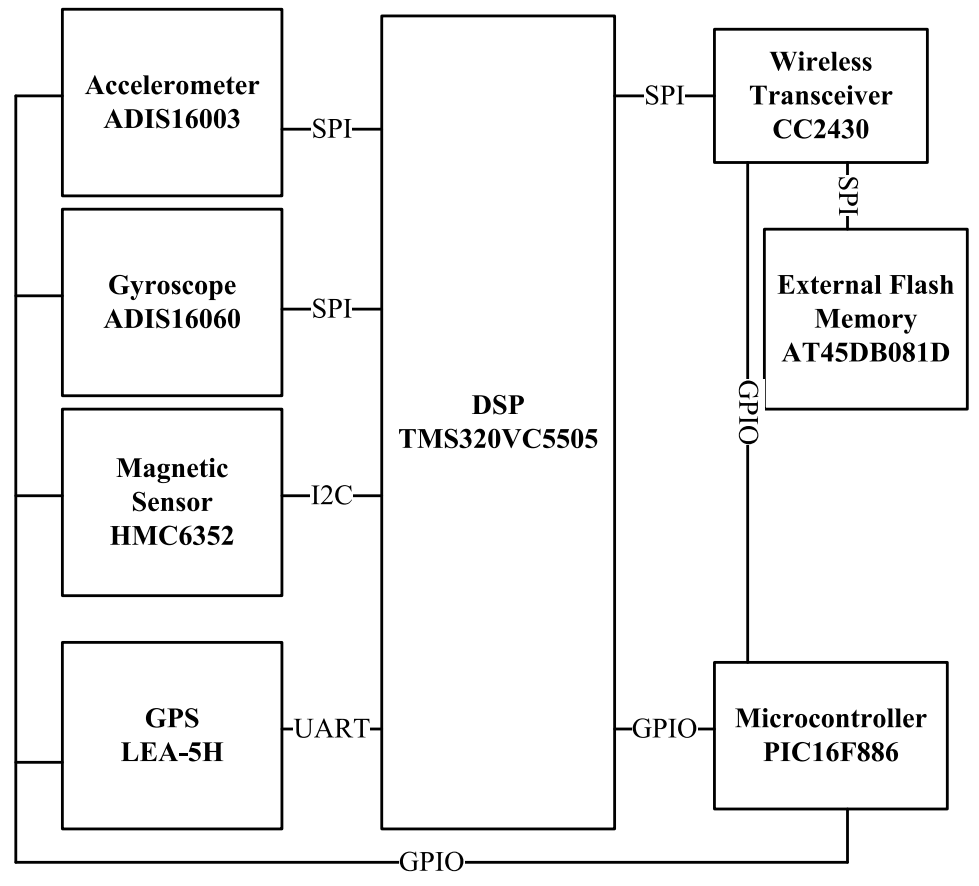

Figure 2.1 The architecture of the proposed system. 
In the above system architecture, a radio transceiver is equipped to make the system compatible with wireless sensor networks. A reduce set of MEMS INS, which consists of a 2-axis accelerometer and a single-axis gyroscope, is used to provide the acceleration and the angular rate required by the integrated system. GPS continuously provides reliable positional information. The magnetic sensor measures the heading angle. A single DSP chip is programmed to process GPS data and carry out the navigation computation. A PIC MCU is used to control the power supply and accomplish the motion detection (see Section 4.3.2.1). A brief review of WSN, GPS and INS is given by the following sections.

\subsection{Overview of WSN and ZigBee}

A wireless sensor network (WSN) consists of a large number of battery-powered and self-organizing sensor nodes, which are spatially distributed to monitor physical or environmental conditions. Those sensors can be deployed anywhere, even in inaccessible areas. There are a wide range of WSN applications such as environment monitoring, landslide detection, smart homes, precision agriculture, battle surveillance, etc. However, in many applications, the battery equipped on each sensor node is not replaceable, which makes the power constraint extremely tight.

One of many significant options for WSN is the ZigBee technology. The ZigBee is an open global standard which is built on top of the IEEE 802.15.4-2003 standard for Wireless Personal Area Networks (WPANs). The IEEE 802.15.4 standard defines the physical and MAC layers, whereas ZigBee defines the network and application layers. A typical ZigBee network is a multi-hop network that enables low power consumption, low cost and low data rate for short-range wireless connections 
between battery-powered devices[42]. The ZigBee system structure is shown in Figure 2.2. ZigBee standardizes both the network and the application layer:

- Network (NWK) layer

The network layer ensures the proper operation of the MAC layer and provides an interface to the application layer. This layer establishes the network, as well as discovers nodes, and permits them to join or leave the network. It supports three different network topologies: star, tree and peer to peer.

- Application layer

The APL layer is composed of three sublayers, which are shown in Figure 2.2. Application Support Sublayer (APS) is mainly responsible for interfacing and controlling devices, address mapping, and message transmission between devices. The Zigbee Device Object (ZDO) is responsible for defining the logical type of the device (i.e. ZC, ZR or ZED), and discovering new devices in the network and identifying their application services. The application framework is at the top of the APL layer, which provides an execution environment for application objects to send and receive data. The application objects, which are defined by the manufacturer, implement various applications such as light switch, heating control or security control, etc.

A typical ZigBee sensor network is composed of sensor nodes, routers and sink nodes. A certain amount of sensor nodes are geographically distributed and deployed within the maximum RF range of fixed relay nodes (routers in the network), in self-organized style. After the primary data processing, the location information of each sensor node will be transmitted through the distributed network with multi-hop routing. Once the data reach the sink node, they can be sent to the manage node through the 
Internet and monitored by the terminal user. The architecture of such a wireless sensor network is shown as Figure 2.3.

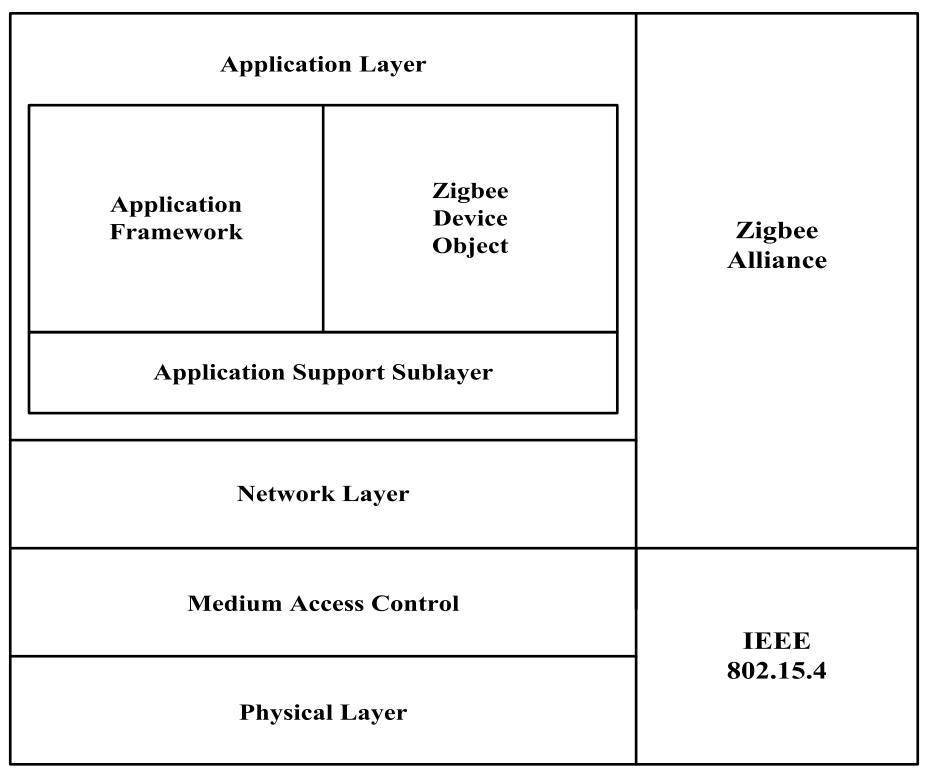

Figure 2.2 Diagram of ZigBee structure.

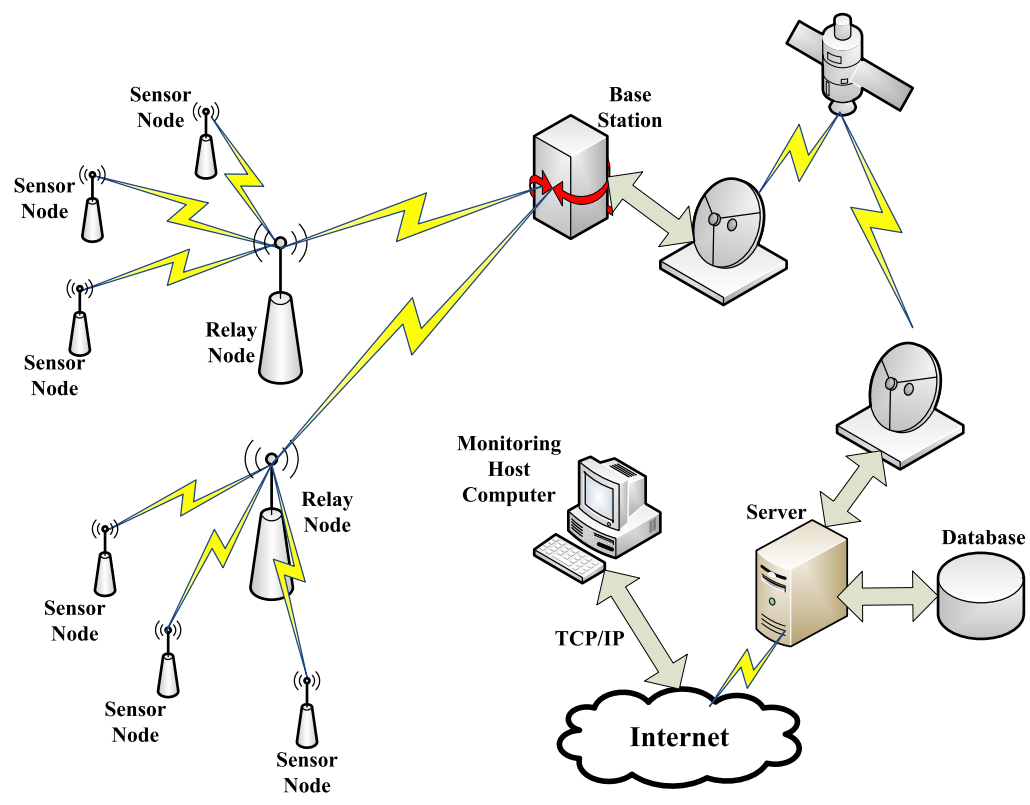

Figure 2.3 The architecture of a typical wireless sensor network. 


\subsection{Overview of the Global Positioning System}

Developed by the U.S. Department of Defense (DoD), the Global Positioning System (GPS) is well known as an all-weather, worldwide, satellite-based radio-navigation system that comprises three segments: the Space Segment, the Control Segment, and the User Segment. The GPS signal is broadcasted at two different carrier frequencies: the primary frequency at $1575.42 \mathrm{MHz}$ (L1) and the secondary frequency at 1227.6 MHz (L2). Signals are encoded using Code Division Multiple Access (CDMA) so that each signal is distinguishable from others based on its unique spread code for each satellite. L1 is modulated by two types of Pseudo-Random Noise (PRN) codes: the CoarseAcquisition (C/A) code at $1.023 \mathrm{MHz}$, which is for civilian use, and the Precise (P) code at 10.23 $\mathrm{MHz}$, which is only for military use. L2 is only modulated by the P code.

In general, the current available GPS signal consists of three types of measurements: pseudorange (a pseudo-range is an estimate of the distance between the GPS receiver and a navigation satellite before being error corrected), carrier phase and Doppler measurements. Pseudo-range measurements are obtained by comparing the PRN codes transmitted from the satellites with the replica PRN codes generated by the receiver to determine the time shift required for the signal to propagate from the satellite to the receiver. The reason why it is termed as pseudo-range instead of range is that the clocks of the satellites and the receiver are not synchronized and the measurements contain clock biases. Since there are four unknown parameters (three coordinates and the clock bias) in the system equations, it is necessary to have at least four satellites calculate the receiver position. Carrier phase measurements are used when a high degree of precision is required in some applications such as geodetic surveying. The Doppler frequency represents the frequency shift caused 
by the rate of change of the carrier phase measurements. It can be used to calculate the relative velocity between the receiver and the satellite. Similar to the case of pseudo-range measurements, the clock bias is also considered to be an unknown parameter in the velocity calculation. Therefore, at least four Doppler measurements are necessary to compute the velocity of the receiver.

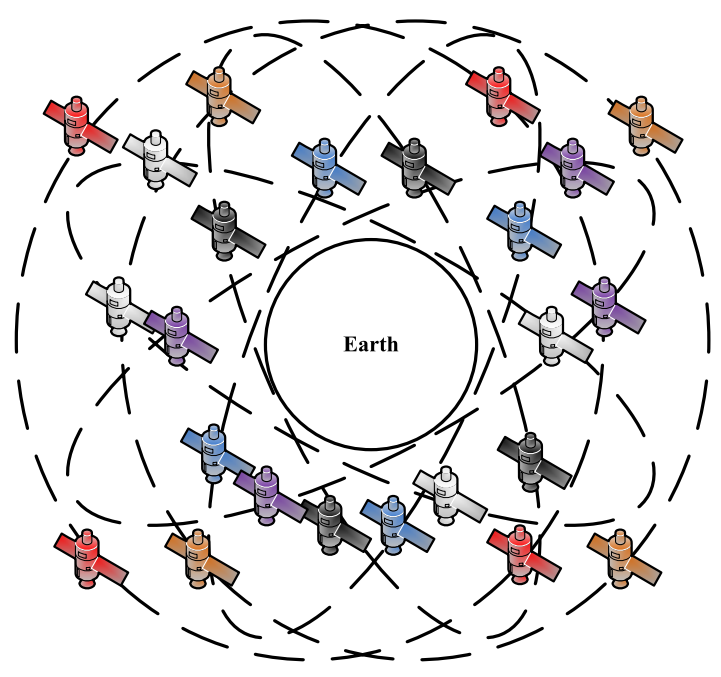

Figure 2.4 Illustration of GPS constellation.

\subsection{Overview of the Inertial Navigation System}

An Inertial Navigation System is an autonomous Dead Reckoning (DR) system that usually combines accelerometers and gyroscopes to provide position and velocity by measuring the accelerations and angular rates applied to the system's inertial frame. Other types of DR sensors may also be used in INS such as the compass, odometer, inclinometer, altimeter, etc. Unlike the GPS that requires external signals to provide positional information, INS is self-contained and immune to jamming and deception, regardless of the operational environment. However, the INS measurements are corrupted by system errors such as inertial alignment errors and sensor errors [17]. Generally there are two 
types of INS: these are classified by the way their sensor axes fix, and are called the gimbaled and strap-down systems. The strap-down system is used in this thesis.

The navigation computation process is briefly introduced in the following sections.

\subsubsection{Mechanization and Quaternion Transformation}

The mechanization equations convert the IMU measurements---specific forces from accelerometers and angular velocities from gyroscopes---in the body frame into navigation information (position, velocity and attitude) in the local level frame where the data fusion and navigation calculation are performed.

The low-cost MEMS sensor suffers from deterministic and stochastic errors that will be presented in Chapter 3. The sensor errors in the raw measurements need to be compensated before the mechanization calculation is performed. After eliminating the sensor errors from the raw measurements, the angular rates in the body frame are integrated to obtain the new orientation of the

INS by updating the rotation matrix $\mathbf{R}_{\mathbf{b}}^{\mathbf{l}}$ from b-frame to local level frame by using the quaternion approach. The angular rate measurement contains both the rotation generated by the INS and the Earth rotation. The earth rotation rate should be converted into the b-frame and removed from the gyro-sensed angular rates. The next step of the IMU data integration is to rotate the specific force measurement from the body frame to the local level frame. The specific force measurement $f$ is combined by the gravity $g$ and the INS acceleration a, which is given by:

$$
\mathbf{f}=\mathbf{a}-\mathbf{g}
$$


After removing the gravity component, the INS acceleration is integrated to calculate the INS velocities and positions in the local level frame.

The l-frame inertial navigation equations can be expressed as follows:

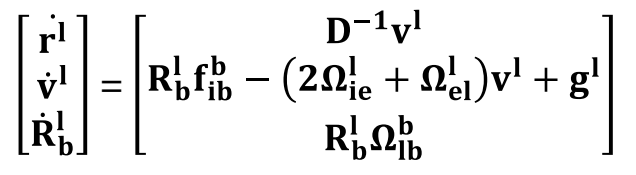

where,

the dot (.) represents the time derivative,

' 1 ' and ' $b$ ' denote the local level frame and b-frame,

$\mathbf{r}^{\mathrm{l}}, \mathbf{v}^{\mathbf{l}}$ and $\mathbf{g}^{\mathrm{l}}$ is the position vector $\left(\mathrm{r}^{\mathrm{l}}=[\varphi, \lambda, \mathrm{h}]^{\mathrm{T}}\right)$, velocity vector $\left(\mathrm{v}^{\mathrm{l}}=\left[\mathrm{v}^{\mathrm{E}}, \mathrm{v}^{\mathrm{N}}, \mathrm{v}^{\mathrm{U}}\right]^{\mathrm{T}}\right)$ and the gravity vector respectively,

$\varphi, \lambda$ and $\mathrm{h}$ is the geodetic latitude, geodetic longitude and geodetic altitude respectively,

$\boldsymbol{\Omega}_{\mathbf{e l}}^{\mathbf{l}}$ is a skew-symmetric matrix of the INS angular rate and $\boldsymbol{\omega}_{\mathrm{el}}^{\mathbf{l}}=[-\dot{\varphi}, \dot{\lambda} \cos \varphi, \dot{\lambda} \sin \varphi]^{\mathrm{T}}$,

$\boldsymbol{\Omega}_{\mathbf{i e}}^{\mathbf{l}}$ is a skew-symmetric matrix of Earth rotation angular rate $\omega_{e}$ and $\boldsymbol{\omega}_{\mathbf{i e}}^{\mathbf{l}}=\mathbf{R}_{\mathbf{e}}^{\mathbf{l}} \boldsymbol{\omega}_{\mathbf{i e}}^{\mathbf{e}}=[0$, $\left.\omega_{\mathrm{e}} \cos \varphi, \omega_{\mathrm{e}} \sin \varphi\right]^{\mathrm{T}}$,

$\mathbf{R}_{\mathrm{b}}^{1}$ is the rotation matrix from the b-frame to the 1-frame,

$$
\begin{aligned}
& \Omega_{\mathrm{lb}}^{b}=\Omega_{\mathrm{ib}}^{\mathrm{b}}-\Omega_{\mathrm{ie}}^{\mathrm{b}}-\Omega_{\mathrm{el}}^{\mathrm{b}} \text { and } \boldsymbol{\omega}_{\mathrm{ie}}^{\mathrm{b}}=\mathbf{R}_{\mathrm{l}}^{\mathrm{b}} \boldsymbol{\omega}_{\mathrm{ie}}^{\mathrm{l}}, \boldsymbol{\omega}_{\mathrm{el}}^{\mathrm{b}}=\mathbf{R}_{\mathrm{l}}^{\mathrm{b}} \boldsymbol{\omega}_{\mathrm{el}}^{\mathrm{l}} \text {, and } \\
& \mathbf{D}^{-1}=\left[\begin{array}{ccc}
0 & \frac{1}{M+h} & 0 \\
\frac{1}{(N+h) \cos \varphi} & 0 & 0 \\
0 & 0 & 1
\end{array}\right]
\end{aligned}
$$


In the above equations, $\boldsymbol{\omega}_{\mathbf{i b}}^{\mathbf{b}}=\left[\omega_{\mathrm{x}}, \omega_{\mathrm{y}}, \omega_{\mathrm{z}}\right]^{\mathbf{T}}$ and $\mathbf{f}_{\mathbf{i b}}^{\mathbf{b}}=\left[\mathrm{f}_{\mathrm{x}}, \mathrm{f}_{\mathrm{y}}, \mathrm{f}_{\mathrm{z}}\right]^{\mathbf{T}}$ are measured by 3 gyroscopes and 3-axis accelerometer respectively. The skew-symmetric matrix $\boldsymbol{\Omega}_{\mathbf{p q}}^{\mathbf{r}}$ corresponding to the vector $\boldsymbol{\omega}_{\mathbf{p q}}^{\mathbf{r}}=\left[\omega_{1}, \omega_{2}, \omega_{3}\right]^{\mathbf{T}}$ is defined as:

$$
\Omega_{p q}^{r}=\left[\begin{array}{ccc}
0 & -\omega_{3} & \omega_{2} \\
\omega_{3} & 0 & -\omega_{1} \\
-\omega_{2} & \omega_{1} & 0
\end{array}\right]
$$

The algorithmic flow diagram of the l-frame mechanization is shown in Figure 2.5.

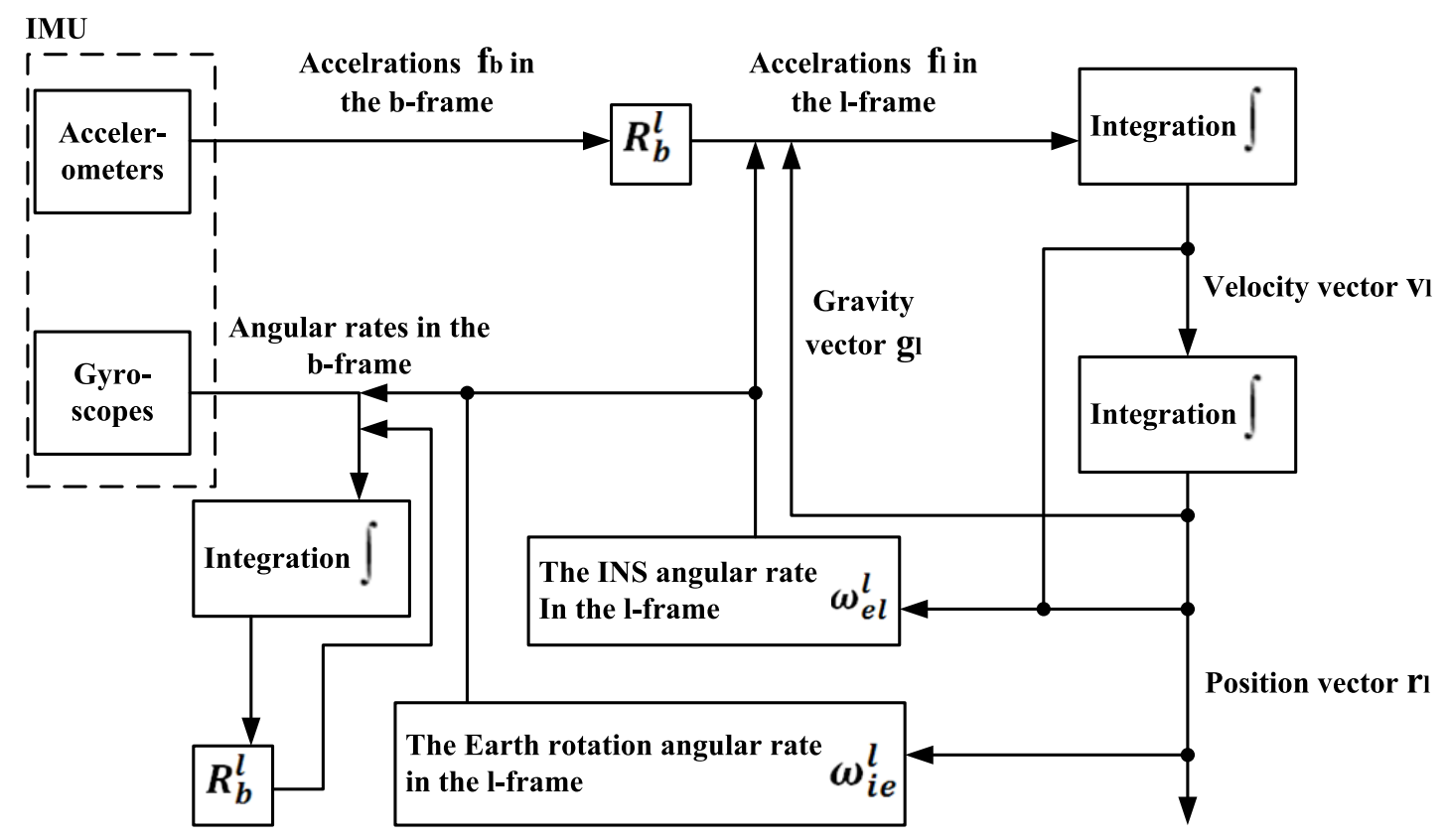

Figure 2.5 l-frame mechanization flow algorithm.

The attitude of a rotating body in the b-frame with respect to a reference frame (i.e. the local level frame) can be expressed by using different attitude parameterization techniques, such as Euler angles, quaternion, and DCM. Due to its computational efficiency and simplicity, the quaternion approach is utilized in this thesis to update the rotation matrix $\mathbf{R}_{\mathrm{b}}^{\mathrm{l}}$, by integrating the angular velocities of body rotation measured by gyroscopes. 
The quaternion $\mathbf{q}$ is a vector with four quaternion parameters. Hamilton's quaternion is defined as a hyper-complex number, which is represented as follows [18]:

$$
\mathbf{q}=\mathbf{q}_{\mathbf{0}}+\mathbf{q}_{1} \cdot \mathbf{i}+\mathbf{q}_{2} \cdot \mathbf{j}+\mathbf{q}_{3} \cdot \mathbf{k}
$$

where,

$\mathrm{q}_{0}, \mathrm{q}_{1}, \mathrm{q}_{2}, \mathrm{q}_{3}$ are real numbers,

set $[1, i, j, k]$ forms the vector basis for a quaternion vector space and $\mathrm{i}^{2}=\mathrm{j}^{2}=\mathrm{k}^{2}=\mathrm{ijk}=$ $-1$

Quaternion transformation from one coordinate frame to another is only effected by a single rotation vector $\Theta[19]$ :

$$
\mathbf{q}=\left[\begin{array}{l}
\mathbf{q}_{0} \\
\mathbf{q}_{1} \\
\mathbf{q}_{2} \\
\mathbf{q}_{3}
\end{array}\right]=\left[\begin{array}{c}
\cos (\Theta / 2) \\
\left(\frac{\Theta_{\mathrm{x}}}{\Theta}\right) \sin (\Theta / 2) \\
\left(\frac{\Theta_{\mathrm{y}}}{\Theta}\right) \sin (\Theta / 2) \\
\left.\left(\frac{\Theta_{\mathrm{z}}}{\Theta}\right) \sin (\Theta / 2)\right)
\end{array}\right]
$$

where $\Theta_{x}, \Theta_{y}$ and $\Theta_{z}$ are three components of the rotation angle $\Theta$ and

$$
\Theta=\sqrt{\Theta_{\mathrm{x}}^{2}+\Theta_{\mathrm{y}}^{2}+\Theta_{\mathrm{z}}^{2}}
$$

The definition of the four quaternion components implies that $q_{0}^{2}+q_{1}^{2}+q_{2}^{2}+q_{3}^{2}=1$, which means there are only three independent quaternion parameters and Equation (2.7) can be used to normalize the quaternion operations.

The relationship between the DCM $\mathbf{R}_{\mathrm{b}}^{\mathrm{l}}$ and the quaternion parameters is represented as follows:

$$
\mathbf{R}_{\mathbf{b}}^{1}=\left[\begin{array}{ccc}
\mathbf{q}_{0}^{2}+\mathbf{q}_{1}^{2}-\mathbf{q}_{2}^{2}-\mathbf{q}_{3}^{2} & 2\left(\mathbf{q}_{1} \mathbf{q}_{2}-\mathbf{q}_{0} \mathbf{q}_{3}\right) & 2\left(\mathbf{q}_{0} \mathbf{q}_{2}+\mathbf{q}_{1} \mathbf{q}_{3}\right) \\
2\left(\mathbf{q}_{1} \mathbf{q}_{2}+\mathbf{q}_{0} \mathbf{q}_{3}\right) & \mathbf{q}_{0}^{2}-\mathbf{q}_{1}^{2}+\mathbf{q}_{2}^{2}-\mathbf{q}_{3}^{2} & 2\left(\mathbf{q}_{2} \mathbf{q}_{3}-\mathbf{q}_{0} \mathbf{q}_{1}\right) \\
2\left(\mathbf{q}_{1} \mathbf{q}_{3}-\mathbf{q}_{0} \mathbf{q}_{2}\right) & 2\left(\mathbf{q}_{2} \mathbf{q}_{3}+\mathbf{q}_{0} \mathbf{q}_{1}\right) & \mathbf{q}_{0}^{2}-\mathbf{q}_{1}^{2}-\mathbf{q}_{2}^{2}+\mathbf{q}_{3}^{2}
\end{array}\right]
$$


and the first-order differential equation for the quaternion components is given by:

$$
\dot{\mathbf{q}}=\frac{1}{2} \Omega(\omega) \mathbf{q}
$$

where the skew-symmetric matrix $\boldsymbol{\Omega}(\boldsymbol{\omega})=\left[\begin{array}{cccc}0 & \omega_{\mathrm{z}} & -\omega_{\mathrm{y}} & \omega_{\mathrm{x}} \\ -\omega_{\mathrm{z}} & 0 & \omega_{\mathrm{x}} & \omega_{\mathrm{y}} \\ \omega_{\mathrm{y}} & -\omega_{\mathrm{x}} & 0 & \omega_{\mathrm{z}} \\ -\omega_{\mathrm{x}} & -\omega_{\mathrm{y}} & -\omega_{\mathrm{z}} & 0\end{array}\right]$ and $\boldsymbol{\omega}_{\mathrm{x}}, \boldsymbol{\omega}_{\mathrm{y}}$ and $\boldsymbol{\omega}_{\mathrm{z}}$ are the angular rates of body rotation.

\subsubsection{Estimation Theory and the Kalman Filter}

Estimation is a process of estimating a set of values for a set of parameters based on a set of observations. The mathematical relationship between the observations and the unknown parameters (the measurement equation) can be expressed as a function of time $t$ :

$$
\mathbf{z}(\mathbf{t})=\mathbf{H}(\mathbf{t}) \mathbf{x}(\mathbf{t})+\mathbf{v}(\mathbf{t})
$$

where,

$\mathbf{z}(\mathbf{t})$ is the m-dimensional observation vector,

$\mathbf{H}(\mathbf{t})$ is an mxn matrix which is called the system design matrix,

$\mathbf{x}(\mathbf{t})$ is the $\mathrm{n}$-dimensional parameter state vector, and

$\mathbf{v}(\mathbf{t})$ is the measurement noise.

There is also a process model that describes the parameter dynamics. Similar to Equation (2.10), the process model is usually expressed as follows:

$$
\dot{\mathbf{x}}(\mathbf{t})=\mathbf{F}(\mathbf{t}) \mathbf{x}(\mathbf{t})+\mathbf{G}(\mathbf{t}) \mathbf{w}(\mathbf{t})
$$

where,

the dot (.) denotes a time derivative, 
$\mathbf{F}(\mathbf{t})$ is an $\mathrm{n} \times \mathrm{n}$ matrix which is called the system dynamic matrix,

$\mathbf{G}(\mathbf{t})$ is the noise shaping matrix which describes how the noise sources are distributed among the state vector components, and

$\mathbf{w}(\mathbf{t})$ is the process driving noise.

The Kalman filter is a recursive state estimator in which the optimal estimate in the least squares sense of the actual state vector is calculated by a series of prediction and measurement update processes. As discussed in Section 1.2, the Kalman filter is a common tool to transfer the GPS and INS measurements into positions, velocities and attitudes. In GPS/INS applications, the inertial sensor measurements are obtained at discrete points in time, and the parameter state vector is estimated in discrete time intervals. Therefore, a discrete time adaption of Equation (2.11) is required as follows:

$$
\mathbf{x}_{\mathrm{k}+\mathbf{1}}=\mathbf{F}_{\mathrm{k}+\mathbf{1}, \mathrm{k}} \mathbf{x}_{\mathrm{k}}+\mathbf{G}_{\mathrm{k}} \mathbf{W}_{\mathbf{k}}
$$

where,

$\mathrm{F}_{\mathrm{k}+1, \mathrm{k}}$ is the state transition matrix between time $\mathbf{t}_{\mathbf{k}}$ and $\mathbf{t}_{\mathbf{k}+\mathbf{1}}$,

$\mathrm{x}_{\mathrm{k}}$ is the parameter state vector at time $\mathbf{t}_{\mathrm{k}}$,

Similarly, the measurement model of a discrete-time controlled process is expressed as:

$$
\mathbf{z}_{\mathbf{k}}=\mathbf{H}_{\mathbf{k}} \mathbf{x}_{\mathbf{k}}+\mathbf{v}_{\mathbf{k}}
$$

In GPS/INS navigation applications, sensor measurements are obtained at discrete times. If we let $\left\{x_{k}\right\}$ be the state vector at those discrete times, then $x_{k}$ can be obtained in the following form:

$$
x_{k}=\Phi_{k-1} x_{k-1}+G w_{k-1}
$$


where $\Phi$ is the state transition matrix.

Equation (2.15) is the 9-state vector used in the EKF for navigation computation and Equation (2.16) shows the corresponding state transition matrix [20]:

$$
\begin{gathered}
x=\left[\delta \varphi, \delta \lambda, \delta h, \delta v_{e}, \delta v_{n}, \delta v_{u}, \delta p, \delta r, \delta y w\right]^{T} \\
\Phi_{9 \times 9}=\left[\begin{array}{lll}
\Phi_{3 \times 3}^{1} & \Phi_{3 \times 3}^{2} & \Phi_{3 \times 3}^{3} \\
\Phi_{3 \times 3}^{4} & \Phi_{3 \times 3}^{5} & \Phi_{3 \times 3}^{6} \\
\Phi_{3 \times 3}^{7} & \Phi_{3 \times 3}^{8} & \Phi_{3 \times 3}^{9}
\end{array}\right]
\end{gathered}
$$

where,

$$
\begin{aligned}
& \boldsymbol{\Phi}_{\mathbf{3} \times \mathbf{3}}^{\mathbf{1}}=\left[\begin{array}{ccc}
0 & 0 & -\frac{\dot{\varphi}}{M+h} \\
\dot{\lambda} \tan \varphi & 0 & -\frac{\dot{\lambda}}{N+h} \\
0 & 0 & 0
\end{array}\right], \quad \boldsymbol{\Phi}_{\mathbf{3} \times \mathbf{3}}^{\mathbf{2}}=\left[\begin{array}{ccc}
0 & \frac{1}{M+h} & 0 \\
\frac{1}{(N+h) \cos \varphi} & 0 & 0 \\
0 & 0 & 1
\end{array}\right], \boldsymbol{\Phi}_{3 \times \mathbf{3}}^{\mathbf{3}}=\left[\begin{array}{lll}
0 & 0 & 0 \\
0 & 0 & 0 \\
0 & 0 & 0
\end{array}\right], \\
& \boldsymbol{\Phi}_{\mathbf{3} \times \mathbf{3}}^{\mathbf{4}}=\left[\begin{array}{ccc}
2 \omega_{e}\left(v_{u} \sin \varphi+v_{n} \cos \varphi\right)+v_{n} \dot{\lambda} / \cos \varphi & 0 & 0 \\
-v_{e} 2 \omega_{e} \cos \varphi-\dot{\lambda} / \cos \varphi & 0 & 0 \\
-2 \omega_{e} v_{e} \sin \varphi & 0 & 2 g / R
\end{array}\right] \text {, } \\
& \boldsymbol{\Phi}_{3 \times \mathbf{3}}^{\mathbf{5}}=\left[\begin{array}{ccc}
\frac{-\dot{h}}{N+h}+\dot{\varphi} \tan \varphi \frac{M+h}{N+h} & \left(2 \omega_{e}+\dot{\lambda}\right) \sin \varphi & -\left(2 \omega_{e}+\dot{\lambda}\right) \cos \varphi \\
-\left(2 \omega_{e}+\dot{\lambda}\right) \sin \varphi & \frac{-\dot{h}}{M+h} & -\dot{\varphi} \\
\left(2 \omega_{e}+\dot{\lambda}\right) \cos \varphi & 2 \dot{\varphi} & 0
\end{array}\right], \boldsymbol{\Phi}_{3 \times \mathbf{3}}^{\mathbf{6}}=\left[\begin{array}{ccc}
0 & f_{u} & -f_{n} \\
-f_{u} & 0 & f_{e} \\
f_{n} & -f_{e} & 0
\end{array}\right] \text {, } \\
& \boldsymbol{\Phi}_{3 \times 3}^{7}=\left[\begin{array}{ccc}
0 & 0 & -\frac{\dot{\varphi}}{M+h} \\
\omega_{e} \sin \varphi & 0 & \frac{\dot{\lambda} \cos \varphi}{N+h} \\
-\omega_{e} \cos \varphi-\dot{\lambda} / \cos \varphi & 0 & \frac{\dot{\lambda} \sin \varphi}{N+h}
\end{array}\right], \quad \boldsymbol{\Phi}_{3 \times 3}^{\mathbf{8}}=\left[\begin{array}{ccc}
0 & \frac{1}{M+h} & 0 \\
-\frac{1}{N+h} & 0 & 0 \\
-\frac{\tan \varphi}{N+h} & 0 & 0
\end{array}\right] \text {, } \\
& \boldsymbol{\Phi}_{\mathbf{3} \times \mathbf{3}}^{\mathbf{9}}=\left[\begin{array}{ccc}
0 & \left(\omega_{e}+\dot{\lambda}\right) \sin \varphi & -\left(\omega_{e}+\dot{\lambda}\right) \cos \varphi \\
-\left(\omega_{e}+\dot{\lambda}\right) \sin \varphi & 0 & -\dot{\varphi} \\
\left(\omega_{e}+\dot{\lambda}\right) \cos \varphi & \dot{\varphi} & 0
\end{array}\right] \text {. }
\end{aligned}
$$

Equation (2.14) is the discrete-time dynamic model corresponding to the continuous-time model.

Then the covariance matrix of process noise has the form $\mathbf{G Q}_{\mathbf{w}} \mathbf{G}^{\mathbf{T}}$, where $\boldsymbol{Q}_{\boldsymbol{w}}$ is the covariance matrix of the white noise process $\mathbf{w}(\mathrm{t})$ : 


$$
E\left[G w_{k}\left(G w_{i}\right)^{T}\right]=\left\{\begin{array}{cc}
G Q_{w} G^{T}, & i=k \\
0, & i \neq k
\end{array}\right.
$$

The Kalman filter algorithm is a two-step process, i.e. prediction and update. Figure 2.6 provides a complete description of the two-step Kalman filter operations.

The optimum combination of measurement and estimated state vector at time $\mathrm{k}$ is obtained from:

$$
\widehat{\boldsymbol{x}}_{k}=\widehat{\boldsymbol{x}}_{\boldsymbol{k}}^{-}+K_{k}\left(\mathbf{z}_{k}-\boldsymbol{H}_{\boldsymbol{k}} \widehat{\boldsymbol{x}}_{\boldsymbol{k}}^{-}\right)
$$

where,

The hat $\left({ }^{\wedge}\right)$ denotes estimate,

the superscript minus (-) denotes the estimate prior to the measurement at time $\mathrm{k}$,

$\boldsymbol{K}_{\boldsymbol{k}}$ denotes the Kalman gain,

$\widehat{\boldsymbol{x}}_{\boldsymbol{k}}^{-}$denotes the a priori estimate, and

$\boldsymbol{z}_{\boldsymbol{k}}$ represents the measurement vector at time $\mathrm{k}$.

The Kalman filter gain used in the equation is given by:

$$
K_{k}=P_{k}^{-} H_{k}^{T}\left(H_{k} P_{k}^{-} H_{k}^{T}+R_{k}\right)^{-1}
$$

The covariance matrix of estimation uncertainty $\boldsymbol{P}_{\boldsymbol{k}}$ is given by:

$$
\boldsymbol{P}_{\boldsymbol{k}}=\left(\boldsymbol{I}-\boldsymbol{K}_{\boldsymbol{k}} \boldsymbol{H}_{\boldsymbol{k}}\right) \boldsymbol{P}_{\boldsymbol{k}}^{-}
$$

where, $\boldsymbol{P}_{\boldsymbol{k}}^{-}$denotes the error covariance matrix prior to using the updated sensor measurements. 


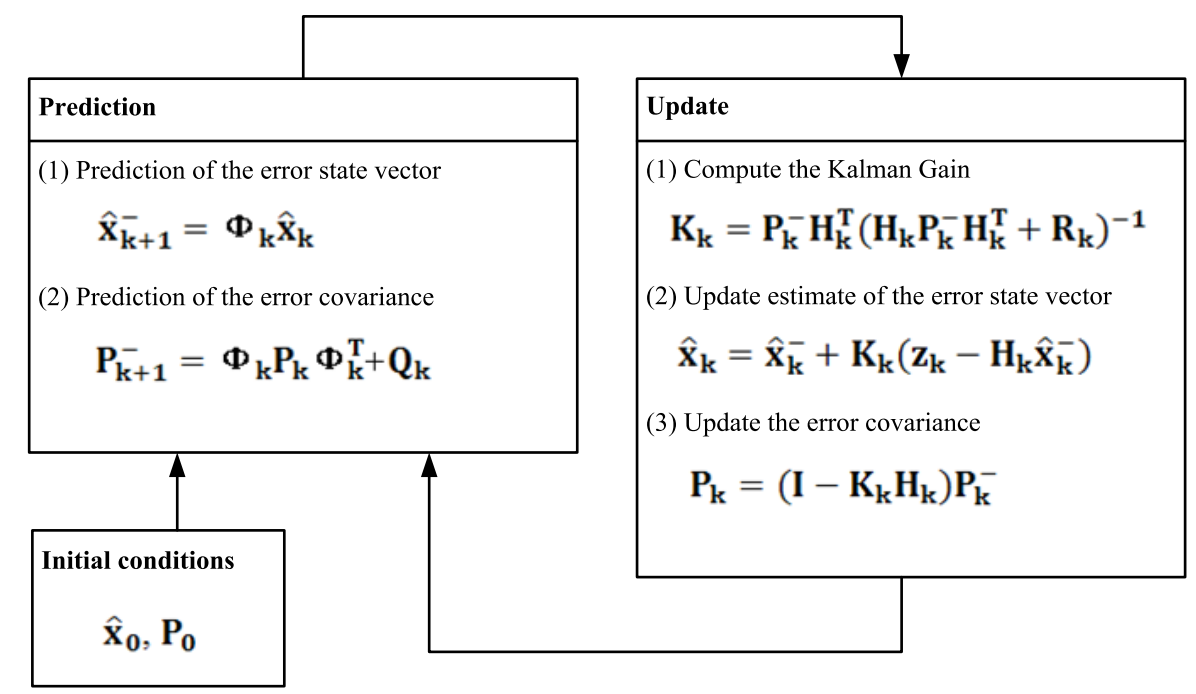

Figure 2.6 A complete picture of the two-step Kalman filter operations.

To recursively calculate the Kalman gain for time $\mathrm{k}+1$, the predictions for the state estimate $\hat{\mathbf{x}}_{\mathbf{k}+\mathbf{1}}^{-}$ and its associated error covariance matrix $\mathbf{P}_{\mathbf{k}+1}^{-}$at the next step (epoch $\left.\mathrm{k}+1\right)$ are given by:

$$
\begin{gathered}
\hat{\mathbf{x}}_{\mathbf{k}+1}^{-}=\Phi_{\mathbf{k}} \hat{\mathbf{x}}_{\mathbf{k}} \\
\mathbf{P}_{\mathbf{k}+\mathbf{1}}^{-}=\Phi_{\mathbf{k}} \mathbf{P}_{\mathbf{k}} \Phi_{\mathbf{k}}^{\mathrm{T}}+\mathbf{Q}_{\mathbf{k}}
\end{gathered}
$$

where, $\mathbf{E}\left[\mathrm{w}_{\mathrm{k}} \mathrm{v}_{\mathrm{i}}^{\mathrm{T}}\right]=\mathbf{0}$, for all $\mathbf{k}$ and $\mathbf{i}$.

And, for the error covariance matrix $\mathbf{P}_{\mathbf{k}}$,

$$
\mathbf{P}_{\mathbf{k}}^{-}=\mathbf{E}\left[\left(\mathbf{x}_{\mathbf{k}}-\hat{\mathbf{x}}_{\mathbf{k}}^{-}\right)\left(\mathbf{x}_{\mathbf{k}}-\hat{\mathbf{x}}_{\mathbf{k}}^{-}\right)^{\mathbf{T}}\right]
$$

The random process to be estimated can be modeled in the form

$$
\mathbf{x}_{\mathbf{k}+1}=\Phi_{\mathbf{k}} \mathbf{x}_{\mathbf{k}}+\mathbf{w}_{\mathbf{k}}
$$

And the process measurement can be modeled as a linear function of the state vector $\mathbf{x}_{\mathbf{k}}$ while the statistical properties of the additive noise $\mathbf{w}_{\mathbf{k}}$ is given:

$$
\mathbf{z}_{\mathbf{k}}=\mathbf{H}_{\mathbf{k}} \mathbf{x}_{\mathbf{k}}+\mathbf{v}_{\mathbf{k}}
$$


where,

$\mathbf{H}_{\mathbf{k}}$ is the measurement sensitivity matrix (observation matrix) at time k, and

$\mathbf{v}_{\mathbf{k}}$ denotes the additive measurement noise which is the electronic noise generated by sensors.

$\mathbf{R}_{\mathbf{k}}$ represents the covariance matrix of sensor noise $\mathbf{v}_{\mathbf{k}}$ as follows:

$$
\mathbf{E}\left[\mathbf{v}_{\mathbf{k}} \mathbf{v}_{\mathbf{i}}^{\mathrm{T}}\right]= \begin{cases}\mathbf{R}_{\mathbf{k}}, & \mathbf{i}=\mathbf{k} \\ \mathbf{0}, & \mathbf{i} \neq \mathbf{k}\end{cases}
$$

\subsubsection{GPS/INS Integration Techniques}

There are two main integration strategies applied to GPS/INS integration at the software level using the KF algorithm: loosely coupled integration and tightly coupled integration.

\subsubsection{Loosely Coupled Integration}

With the loosely coupled integration algorithm, the GPS raw measurements (pseudo-range measurements, carrier phase measurements and Doppler measurements) are first fed to a GPS-only Kalman filter to calculate the GPS derived positions and velocities before being used as observation updates to aid the INS for the navigation KF. It is common for the loosely coupled approach to use the cascaded scheme to combine GPS positions and velocities with INS raw measurements to form the error residuals. These are then used by the navigation KF to estimate the observable INS errors such as biases, drifts and misalignment errors. These estimates then feedback to compensate new INS measurements.

Based on different system requirements, the loosely coupled scheme can either be implemented in open-loop or closed-loop configurations. Open-loop implementation does not require feedback from the navigation KF. Therefore, all the IMU measurements and INS-derived navigation 
information are not corrected before being used. This is a simple configuration which is not well suited to low-cost MEMS IMU, for the reason that large navigation errors cannot be compensated for and those errors may degrade the system accuracy quickly without the feedback loop. Based on the fact that MEMS sensors are not very accurate and their errors fluctuate rapidly, real-time feedback proves to be particularly useful to enhance the performance of the integrated navigation system.

Compared with the tight integration strategy, the primary advantage of the loosely coupled integration is that the dimension of the state vectors in the navigation KF is smaller than that in the tightly coupled case [21]. It takes shorter filter convergence time and has a lower computation load. Therefore, the loosely coupled integration approach has been widely applied in various applications due to its flexibility and fast processing time.

The main drawback of loosely coupled integration is that the GPS receiver cannot compute positions and velocities when the number of tracked GPS satellites is less than four. Therefore, INS should work in a stand-alone mode under harsh GPS conditions. Another disadvantage is that there are two independent Kalman filters in the loose integration scheme, which means the process noise is added to both the GPS Kalman filter and the navigation Kalman filter.

\subsubsection{Tightly Coupled Integration}

Tightly coupled integration is another common strategy used to fuse navigation data provided by the GPS/INS sensing pair [22-24]. In tight integration strategy, only one single integrated filter is used for the data fusion. Without the GPS filter, raw GPS measurements are fed to the navigation filter 
directly, instead of GPS positions and velocities. In this case, the GPS receiver clock bias and drift is added into the filter error states and used to correct the GPS pseudo-range and Doppler measurements.

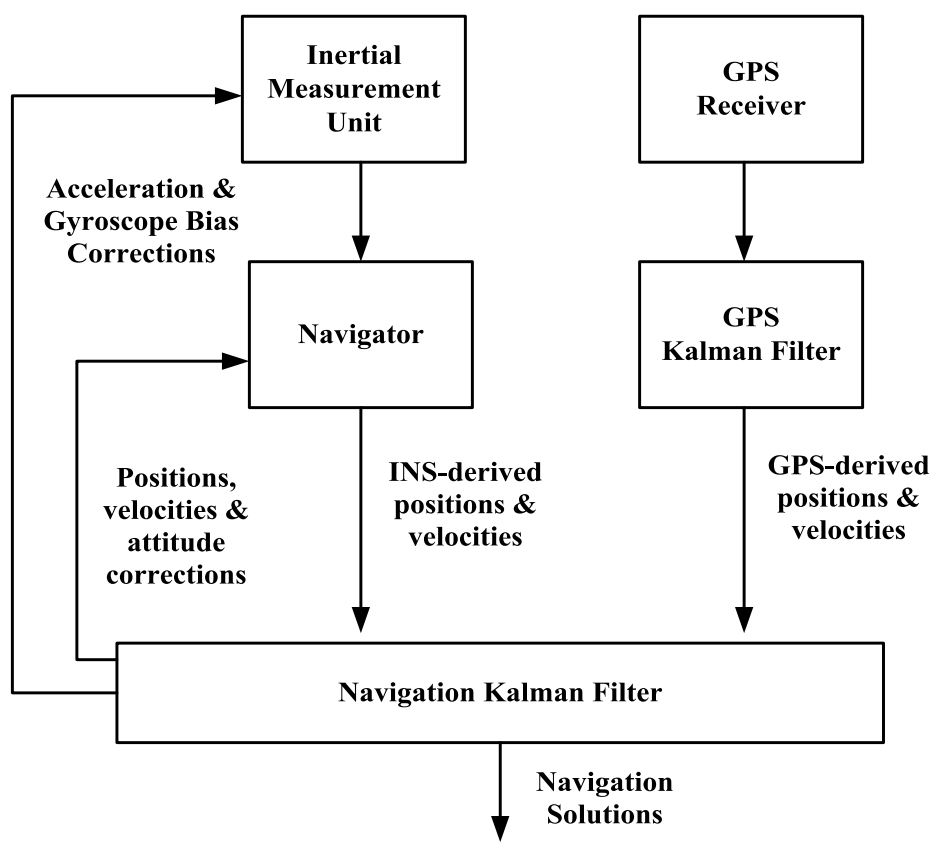

Figure 2.7 Loosely coupled GPS/INS integration.

Consequently, the output of this navigation filter can be used to aid the GPS receiver in tracking satellites.

Clearly, compared with loosely coupled integration, tightly coupled integration is more tolerant of GPS outages, because it allows for efficient use of GPS pseudo-range measurements when the number of tracked navigation satellite is less than 4 . Moreover, the process noise is only added to a single Kalman filter, which improves GPS data filtering. However, since the navigation filter needs an additional component, i.e. GPS pseudo-range measurements, in the system model, the state vector 
size of tight integration is larger than that of loose integration. It leads to longer convergence time for error estimates and increased computational loads. Another negative feature of the tight integration scheme is the indirect GPS measurements which weaken the degree of the observability of the state vector more than the loose integration strategy.

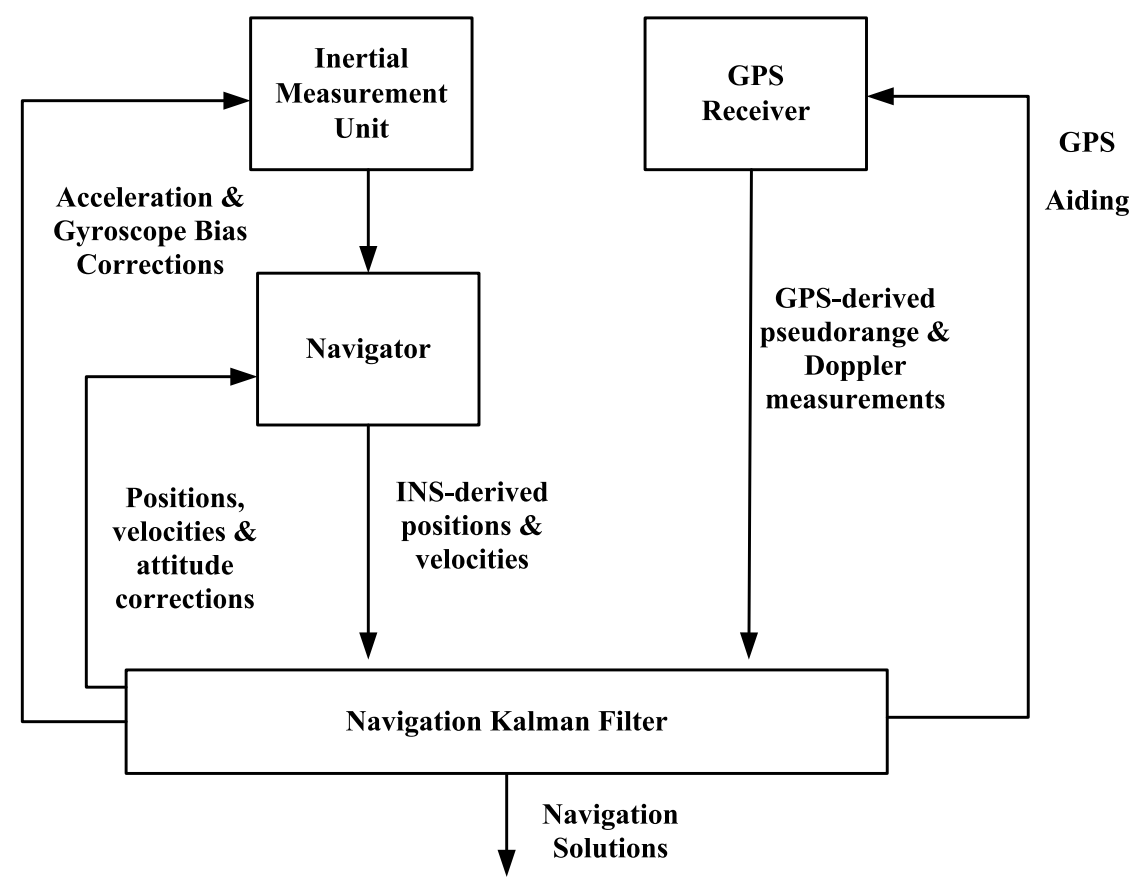

Figure 2.8 Tightly coupled GPS/INS integration. 


\section{Chapter 3}

\section{Accuracy Improvement Methods for Low Cost Systems}

MEMS inertial sensors are small, compact and cost effective, which makes them efficient and affordable in most civilian navigation applications. The output of MEMS-based IMU is accurate in a short time period. However, they are significantly affected by various error sources which accumulate and grow over time, resulting in large position and orientation errors. Hence, it is necessary to calibrate these errors and remove them from the raw data.

\subsection{INS Error Sources and Properties}

The error of inertial sensors can be divided into two categories: deterministic errors and stochastic errors. Deterministic error sources include bias offsets, scale factor instabilities and nonorthogonalities. These sources are usually determined by specific calibration methods in a laboratory environment. The stochastic errors include sensor bias drifts and scale factor drifts. In the case of the gyroscopes, the angular rate measurements given by gyroscope sensors can be described by the true input angular rate and the error terms in the following equation in which the bias coefficients and the anisoelastic bias coefficient are neglected and only the first order scale factor is considered [17]:

$$
l_{\omega_{x}}=\left(1+s_{\omega_{x}}\right) \omega_{x}+m_{\omega_{x y}} \omega_{y}+m_{\omega_{x z}} \omega_{z}+b_{\omega_{x}}+n_{\omega_{x}}
$$

where,

$l_{\omega_{x}}$ is the $\mathrm{x}$-axis gyroscope measurement 
$\omega_{x}$ is the true angular rate about $\mathrm{x}$ axis,

$\omega_{y}$ and $\omega_{z}$ are the true angular rate about $y$ axis and $z$ axis respectively,

$s_{\omega_{x}}$ is the first order $\mathrm{x}$-axis linear scale factor,

$m_{\omega_{x y}}$ and $m_{\omega_{x z}}$ denote the cross-axis coupling coefficients (i.e. non-orthogonalities),

$b_{\omega_{x}}$ is the $\mathrm{x}$-axis bias offset, and

$n_{\omega_{x}}$ is the sensor bias drift and white noise.

Similarly the accelerometer error model is given by the following equation:

$$
\boldsymbol{l}_{f_{x}}=\left(1+\boldsymbol{s}_{f_{x}}\right) \boldsymbol{f}_{x}+\boldsymbol{m}_{f_{x y}} \boldsymbol{f}_{\boldsymbol{y}}+\boldsymbol{m}_{f_{x z}} \boldsymbol{f}_{z}+\boldsymbol{b}_{f_{x}}+\boldsymbol{n}_{f_{x}}
$$

If $\left(1+s_{\omega_{x}}\right)$ is substituted by $m_{\omega_{x x}}$, based on Equation (3.1), the outputs of a triad of gyroscopes can be expressed in matrix form as follows:

$$
\left[\begin{array}{l}
\boldsymbol{l}_{\omega_{x}} \\
\boldsymbol{l}_{\omega_{y}} \\
\boldsymbol{l}_{\omega_{z}}
\end{array}\right]=\left[\begin{array}{lll}
\boldsymbol{m}_{\omega_{x x}} & \boldsymbol{m}_{\omega_{x y}} & \boldsymbol{m}_{\omega_{x z}} \\
\boldsymbol{m}_{\omega_{y x}} & \boldsymbol{m}_{\omega_{y y}} & \boldsymbol{m}_{\omega_{y z}} \\
\boldsymbol{m}_{\omega_{z x}} & \boldsymbol{m}_{\omega_{z y}} & \boldsymbol{m}_{\omega_{z z}}
\end{array}\right]\left[\begin{array}{c}
\omega_{x} \\
\omega_{y} \\
\omega_{z}
\end{array}\right]+\left[\begin{array}{l}
b_{\omega_{x}} \\
\boldsymbol{b}_{\omega_{y}} \\
\boldsymbol{b}_{\omega_{z}}
\end{array}\right]+\left[\begin{array}{l}
\boldsymbol{n}_{\omega_{x}} \\
\boldsymbol{n}_{\omega_{y}} \\
\boldsymbol{n}_{\omega_{z}}
\end{array}\right]
$$

\subsubsection{Sensor Bias}

The inertial sensor bias is defined as the average of the inertial sensor output over a specified time, measured at specified operating conditions that have no correlation with input acceleration or rotation [25]. Bias of accelerometer is expressed in meters per square second $\left(\mathrm{m} / \mathrm{s}^{2}\right)$ and bias of gyroscope is expressed in degrees per hour $\left({ }^{\circ} / \mathrm{h}\right)$. 
There are generally two parts of the sensor bias: the bias offset and the bias drift. The bias offset is deterministic in nature and stable over a certain period of time if the sensor stays on. The bias offset is negligible for high-end inertial sensors. However, the bias offset in low-cost MEMS sensors cannot remain constant over long periods of time and it varies from one start (switch-on) to another. The bias offset error can be determined by calibration and removed from the raw data. The bias drift refers to the error which accumulates over time [26]. It is random in nature and should be modeled as a stochastic process.

\subsubsection{Scale Factors}

A scale factor is the ratio of a change in the output relative to a change in the input intended to be measured [25]. According to the definition, it can be acquired as the slope of the straight line which is given by using the least-square method to the input-output data. The scale factor is typically expressed in parts per million (PPM). Similar to sensor bias, the scale factor is deterministic in nature

and can be determined by calibration. The scale factor of low-cost IMUs changes from switch-on to switch-on.

\subsubsection{Non-Orthogonalities}

Both the accelerometers and gyroscopes in IMUs should be mounted in orthogonal triads. However, one axis cannot be perfectly orthogonal to the other due to manufacturing errors, so that the measurement from one axis is correlated to the other axes in the body frame. 


\subsection{Inertial Sensor Calibration}

Inertial sensor calibration is a comparison between sensor outputs and reference information to determine the sensor errors like noises, biases, drifts, scale factors and non-orthogonalities.

\subsubsection{Six-Position Static Test}

Generally, standard calibration methods were designed for navigation-grade and tactical-grade inertial sensors in laboratory environments, such as the six-position static test and the angular rate test [17].

The six position test requires a level table on which the inertial system is installed with its three accelerometer axes alternately pointing up and down. When one of the sensitive axes is orthogonal to the level table, the acceleration of gravity is taken as the reference and the specific force measurement can be given using Equation (3.2) [27]:

$$
\begin{gathered}
l_{f}^{u p}=(1+s) g+b \\
l_{f}^{\text {down }}=-(1+s) g+b
\end{gathered}
$$

where,

$l_{f}^{u p}$ and $l_{f}^{\text {down }}$ denotes the accelerometer measurement when it points up and down respectively,

$\mathrm{g}$ is the magnitude of the gravity vector, and

$\mathrm{b}$ and $\mathrm{s}$ are the bias error and first order scale factor of the sensitive axis.

The combination of Equation (3.4) and Equation (3.5) gives: 


$$
\begin{gathered}
b=\left(l_{f}^{u p}+l_{f}^{\text {down }}\right) / 2 \\
s=\left(l_{f}^{\text {up }}-l_{f}^{\text {down }}-2 g\right) / 2 g
\end{gathered}
$$

Equation (3.6) and Equation (3.7) can also be used for the static gyroscope test by taking the earth rotation rate $\boldsymbol{\omega}_{\boldsymbol{e}}$ as reference signal. However, consumer-grade gyros such as MEMS gyros cannot use Equation (3.7) to calculate the scale factor $s$ because the earth rotation rate is completely masked by MEMS sensor noise.

By using the static six-position method to measure the deterministic errors of accelerometers, the Equation (3.3) can be rewritten as [27]:

$$
\left[\begin{array}{l}
\boldsymbol{l}_{f_{x}} \\
\boldsymbol{l}_{f_{y}} \\
\boldsymbol{l}_{f_{z}}
\end{array}\right]=\left[\begin{array}{llll}
\boldsymbol{m}_{f_{x x}} & \boldsymbol{m}_{f_{x y}} & \boldsymbol{m}_{f_{x z}} & \boldsymbol{b}_{f_{x}} \\
\boldsymbol{m}_{f_{y x}} & \boldsymbol{m}_{f_{y y}} & \boldsymbol{m}_{f_{y z}} & \boldsymbol{b}_{f_{y}} \\
\boldsymbol{m}_{f_{z x}} & \boldsymbol{m}_{f_{z y}} & \boldsymbol{m}_{f_{z z}} & \boldsymbol{b}_{f_{z}}
\end{array}\right]\left[\begin{array}{c}
f_{x} \\
\boldsymbol{f}_{y} \\
\boldsymbol{f}_{z} \\
\mathbf{1}
\end{array}\right]+\left[\begin{array}{l}
\boldsymbol{n}_{f_{x}} \\
\boldsymbol{n}_{f_{y}} \\
\boldsymbol{n}_{f_{z}}
\end{array}\right]
$$

The true input specific forces at six positions are given by:

$$
A=\left[\begin{array}{cccccc}
g & -g & 0 & 0 & 0 & 0 \\
0 & 0 & g & -g & 0 & 0 \\
0 & 0 & 0 & 0 & g & -g \\
1 & 1 & 1 & 1 & 1 & 1
\end{array}\right]
$$

The average accelerometer measurements $\boldsymbol{l}_{\boldsymbol{x} \text {-axis down }}$ (and $\boldsymbol{l}_{\boldsymbol{x} \text {-axis up }}$, etc.) are obtained by recording the static sensor outputs of a certain time period (at least 15 minutes) when the inertial system is mounted on the level table with its $\mathrm{x}$-axis pointing down. In this way, the measurement matrix can be expressed in this form:

$$
L=\left[\begin{array}{llllll}
l_{x-a x i s} \text { down } & l_{x-a x i s ~ u p} & l_{y-a x i s d o w n} & l_{y-a x i s u p} & l_{z-a x i s d o w n} & l_{z-a x i s u p}
\end{array}\right]
$$


where $\boldsymbol{l}_{\boldsymbol{x} \text {-axis down }}=\left[\begin{array}{l}l_{f_{x}} \\ l_{f_{y}} \\ l_{f_{z}}\end{array}\right]_{x-\text { axis down }}$ and so on.

By using the least squares method, the deterministic error coefficient matrix $\mathbf{M}$ can be estimated as follows:

$$
M=\left[\begin{array}{llll}
\boldsymbol{m}_{f_{x x}} & \boldsymbol{m}_{f_{x y}} & \boldsymbol{m}_{f_{x z}} & \boldsymbol{b}_{f_{x}} \\
\boldsymbol{m}_{f_{y x}} & \boldsymbol{m}_{f_{y y}} & \boldsymbol{m}_{f_{y z}} & \boldsymbol{b}_{f_{y}} \\
\boldsymbol{m}_{f_{z x}} & \boldsymbol{m}_{f_{z y}} & \boldsymbol{m}_{f_{z z}} & \boldsymbol{b}_{f_{z}}
\end{array}\right]=L \cdot \boldsymbol{A}^{T} \cdot\left(\boldsymbol{A} \boldsymbol{A}^{T}\right)^{-1}
$$

Analogous to the accelerometer case, this estimation method can be also applied to the gyroscope calibration by either using the earth rotation rate for high-grade sensors or using a precise turn table for MEMS sensors. In the later case, the inertial system is fixed on the testing table and rotates with a constant angular rate that serves as the preset reference value $\boldsymbol{\omega}_{\boldsymbol{r}}$. The test needs to perform the rotation in both the clockwise and anti-clockwise directions for each sensitive axis. Then the true input rotating speeds are given by:

$$
\boldsymbol{G}=\left[\begin{array}{cccccc}
\omega_{r} & -\omega_{r} & 0 & 0 & 0 & 0 \\
\mathbf{0} & \mathbf{0} & \omega_{r} & -\omega_{r} & 0 & 0 \\
\mathbf{0} & \mathbf{0} & \mathbf{0} & \mathbf{0} & \omega_{r} & -\omega_{r} \\
1 & 1 & \mathbf{1} & \mathbf{1} & \mathbf{1} & \mathbf{1}
\end{array}\right]
$$

\subsection{Stochastic Sensor Error Modeling}

The stochastic part of the inertial sensor errors generally includes two parts: long-term (lowfrequency) and short-term (high-frequency) components [28]. The high frequency component is characterized by white noise, whereas the low frequency component consists mainly of correlated noise. The wavelet de-noising technique is usually used to de-noise the high-frequency component of 
the inertial sensors prior to processing [29, 30]. In most GPS/INS applications, using white Gaussian noise to model the low-frequency noise component is not adequate. It is a common way to generate the colored (correlated) noise by passing a white noise of zero mean through a shaping filter. The colored noise can be modeled as various random processes such as Auto-Regressive (AR) processes and Gauss-Markov (GM) processes. In Kalman filter implementations for INS/GPS, a frequently used method to shape the correlated noise is the first order GM process, which has a simple mathematical description and fits a large number of physical processes with acceptable accuracy.

A GM process is a stationary Gaussian process which has an exponential autocorrelation. The autocorrelation function of a GM process $\mathrm{X}(\mathrm{t})$ is given as follows:

$$
\mathbf{R}_{\mathbf{x}}(\mathbf{t})=\sigma^{2} \mathbf{e}^{-\boldsymbol{\beta}|\mathbf{t}|}
$$

where,

$\tau=1 / \beta$ is the time constant (correlation time), and

$\sigma^{2}$ is the noise variance of the GM process.

The correlation time $1 / \beta$ is evaluated at $\mathrm{t}=\tau$, i.e.:

$$
R_{x}(\tau)=\sigma^{2} e^{-1}=0.3678 \sigma^{2}
$$

The power spectral density (PSD) function of a GM process is given by:

$$
S_{x}(j \omega)=\frac{2 \sigma^{2} \beta}{\omega^{2}+\beta^{2}}
$$

or

$$
S_{x}(s)=\frac{2 \sigma^{2} \beta}{-s^{2}+\beta^{2}}
$$




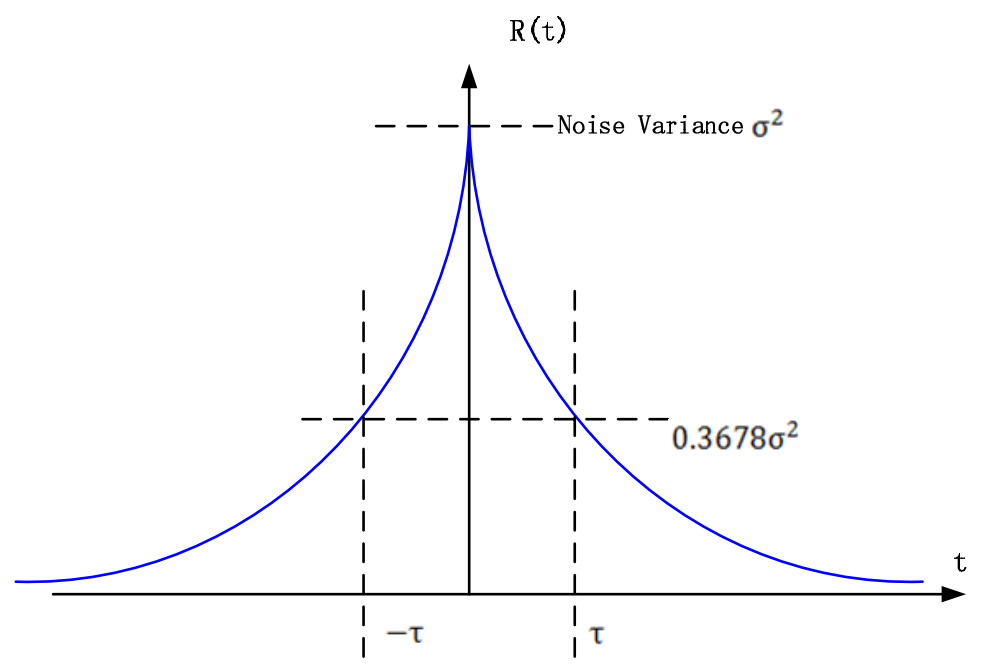

Figure 3.1 Autocorrelation function of the GM process.

Figure 3.1 implies that the correlation between measurement samples becomes weaker as the time-distance between samples increases.

First order Gauss-Markov (GM) process can be expressed by the following first order differential equation [31]:

$$
\dot{\mathbf{x}}(\mathbf{t})=-\frac{1}{\tau} \mathbf{x}(\mathbf{t})+\sqrt{\frac{2 \sigma^{2}}{\tau}} \mathbf{W}(\mathbf{t})
$$

where,

$\mathrm{w}(\mathrm{t})$ is a white noise of zero-mean, and

$\mathrm{x}(\mathrm{t})$ denotes a time-correlated (or colored) noise modeled by the first order GM process.

The discrete-time form of the $1^{\text {st }}$ order GM process is given by (at $\Delta \mathrm{t}$ sampling interval):

$$
\mathbf{x}_{k}=(I-\beta \Delta t) x_{k-1}+\sqrt{2 \beta \sigma^{2}} \mathbf{w}_{k} \Delta t
$$


Based on Equation (3.18), the shaping filter of the $1^{\text {st }}$ order GM model can be described by

Figure 3.2:

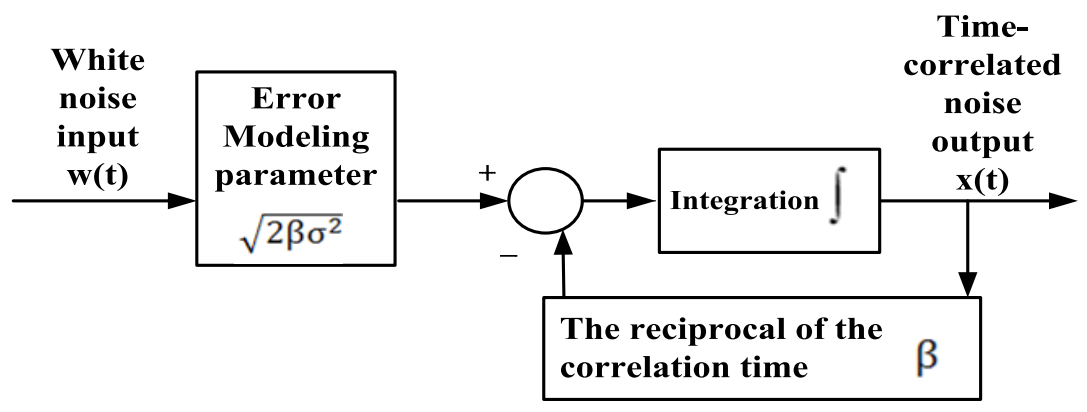

Figure 3.2 Shaping filter of the $1^{\text {st }}$ order GM process.

The first 9 elements of the KF state vector are introduced in Chapter 2. The Kalman filter for the GM model has 15 error states in total and the additional 6 states consist of 3 accelerometer bias drift errors and 3 gyroscope bias drift errors, namely, $\delta f_{x}, \delta f_{y}, \delta f_{z}$ and $\delta \omega_{x}, \delta \omega_{y}, \delta \omega_{z}$ respectively.

From Equation (3.18), the state space form of the 6 inertial sensor random errors can be expressed in the following matrix form:

$$
\left[\begin{array}{c}
\delta \omega_{\mathrm{x}} \\
\delta \omega_{\mathrm{y}} \\
\delta \omega_{\mathrm{z}} \\
\delta f_{\mathrm{x}} \\
\delta f_{\mathrm{y}} \\
\delta f_{\mathrm{z}}
\end{array}\right]_{\mathrm{k}}=G M_{6 \times 6}\left[\begin{array}{c}
\delta \omega_{\mathrm{x}} \\
\delta \omega_{\mathrm{y}} \\
\delta \omega_{\mathrm{z}} \\
\delta f_{\mathrm{x}} \\
\delta f_{\mathrm{y}} \\
\delta f_{\mathrm{z}}
\end{array}\right]_{\mathrm{k}-1}+\left[\begin{array}{c}
\sqrt{2 \beta_{\omega \mathrm{x}} \sigma_{\omega \mathrm{x}}^{2}} \\
\sqrt{2 \beta_{\omega \mathrm{y}} \sigma_{\omega \mathrm{y}}^{2}} \\
\sqrt{2 \beta_{\omega \mathrm{z}} \sigma_{\omega \mathrm{z}}^{2}} \\
\sqrt{2 \beta_{\mathrm{fx}} \sigma_{\mathrm{fx}}^{2}} \\
\sqrt{2 \beta_{\mathrm{fy}} \sigma_{\mathrm{fy}}^{2}} \\
\sqrt{2 \beta_{\mathrm{fz}} \sigma_{\mathrm{fz}}^{2}}
\end{array}\right] w_{\mathrm{k}} \Delta t
$$

where, 


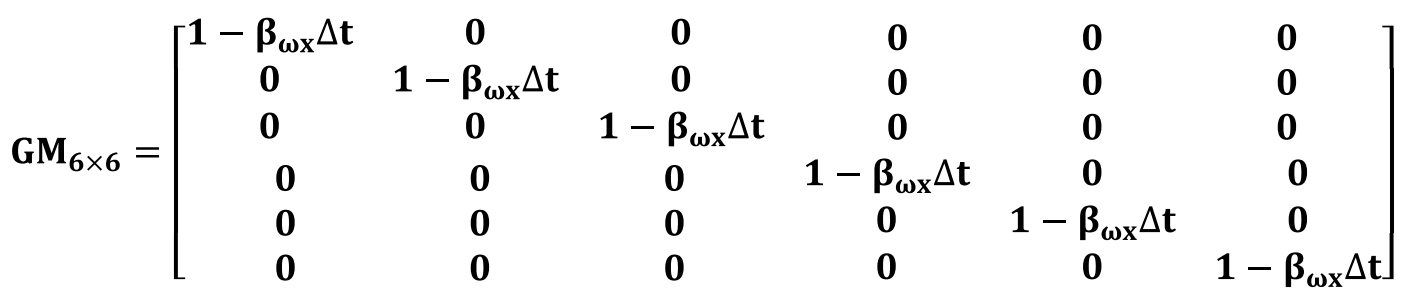

After adding the 6 inertial sensor random errors into the error state vector, the state transition matrix $\Phi_{15 \times 15}$ is given as follows:

$$
\Phi_{15 \times 15}=\left[\begin{array}{ll}
\Phi_{9 \times 9} & R_{b 9 \times 6}^{1} \\
0_{6 \times 9} & G_{6 \times 6}
\end{array}\right]
$$

where,

$$
\begin{aligned}
& \boldsymbol{\Phi}_{\mathbf{9} \times \mathbf{9}} \text { is given by Equation (2.16), } \\
& \mathbf{R}_{\mathbf{b} \times \mathbf{6}}^{\mathbf{l}}=\left[\begin{array}{cc}
\mathbf{0}_{\mathbf{3} \times \mathbf{3}} & \mathbf{0}_{\mathbf{3} \times \mathbf{3}} \\
\mathbf{0}_{\mathbf{3} \times \mathbf{3}} & \mathbf{R}_{\mathbf{b}}^{\mathbf{l}} \\
\mathbf{R}_{\mathbf{b}}^{\mathbf{l}} & \mathbf{0}_{\mathbf{3} \times \mathbf{3}}
\end{array}\right] \text { and } \mathbf{R}_{\mathbf{b}}^{\mathbf{l}} \text { is given by Equation (2.8). }
\end{aligned}
$$

\subsection{Non-Holonomic Constraints}

The Non-Holonomic Constraints (NHC) are originally used to improve the navigation accuracy of land vehicles. The constraints refers to the fact that the vertical velocity and the sideway velocity of a land vehicle is almost zero unless it jumps off the ground or slides on the ground [32]. Therefore, there are two non-holonomic constraints in the body frame that can be used as additional measurement updates to the Kalman filter:

$$
\left\{\begin{array}{l}
\mathbf{v}_{\mathbf{x}}^{\mathbf{b}} \approx \mathbf{0}+\mathbf{n}_{\mathrm{vbx}} \\
\mathbf{v}_{\mathbf{z}}^{\mathbf{b}} \approx \mathbf{0}+\mathbf{n}_{\mathrm{vbz}}
\end{array}\right.
$$

where, $\mathrm{n}_{\mathrm{vbx}}$ and $\mathrm{n}_{\mathrm{vbz}}$ are inertial sensor noises. 
These two non-holonomic constraints may also fit the motion dynamics of other kinds of applications such as animal monitoring and pedestrian navigation. To minimize errors when applying NHC, the IMU should be installed close to the rotational centre of the moving vehicle and the inertial sensors should be accurately aligned to the b-frame.

By using the non-holonomic constraints, we have two sensor measurements in the body frame, which are provided by the accelerometers:

$$
\delta \mathbf{z}_{\text {non-hol }}=\left[\delta \mathbf{v}_{\mathbf{x}}^{\mathbf{b}}, \boldsymbol{\delta} \mathbf{v}_{\mathbf{z}}^{\mathbf{b}}\right]^{\mathbf{T}}
$$

and the velocity errors can be expressed by:

$$
\delta \mathbf{v}^{\mathbf{b}}=\mathbf{R}_{\mathbf{l}}^{\mathbf{b}} \delta \mathbf{v}^{\mathbf{l}}-\mathbf{R}_{\mathbf{l}}^{\mathbf{b}}\left(\mathbf{v}^{\mathbf{l}} \times\right) \varepsilon
$$

where,

$\delta \mathbf{v}^{\mathrm{b}}$ and $\delta \mathbf{v}^{\mathrm{l}}$ are the velocity error vectors in the b-frame and the l-frame respectively,

$\mathbf{R}_{1}^{\mathrm{b}}$ is the rotation matrix from 1-frame to b-frame,

$\times$ denotes cross product,

$(\boldsymbol{\omega} \times) \stackrel{\text { def }}{=}\left[\begin{array}{ccc}0 & -\omega_{3} & \omega_{2} \\ \omega_{3} & 0 & -\omega_{1} \\ -\omega_{2} & \omega_{1} & 0\end{array}\right]$ if $\boldsymbol{\omega}=\left[\omega_{1}, \omega_{2}, \omega_{3}\right]^{\mathbf{T}}$, and

$\boldsymbol{\varepsilon}$ is the attitude error vector, $\boldsymbol{\varepsilon}=[\delta p, \delta r, \delta y w]^{\mathbf{T}}$.

The measurement model of non-holonomic constraints can be written as:

$$
\delta \mathbf{z}_{\text {non-hol }}=\mathbf{H}_{\text {non-hol }} \mathbf{x}+\mathbf{v}
$$

where,

$\mathbf{x}$ is a 15 -state vector of EKF and

$$
\mathbf{x}=\left[\delta \varphi, \delta \lambda, \delta h, \delta v_{e}, \delta v_{n}, \delta v_{u}, \delta p, \delta r, \delta y w, \delta \omega_{x}, \delta \omega_{y}, \delta \omega_{z}, \delta f_{x}, \delta f_{x^{\prime}} \delta f_{z}\right]^{\mathbf{T}}
$$


According to Equation (3.24), the design matrix of non-holonomic constraints $\mathbf{H}_{\text {non-hol }}$ is expressed as:

$$
\mathbf{H}_{\text {non-hol }}=\left[\mathbf{0}_{2,3}, \mathrm{H}_{\text {non-hol1 }}, \mathrm{H}_{\text {non-hol2 }}, \mathbf{0}_{2,6}\right]
$$

where,

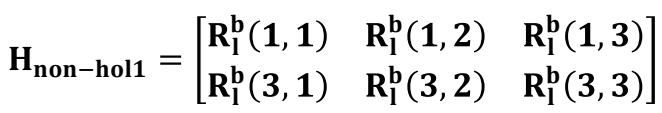

$$
\begin{aligned}
& H_{n o n-h o l 2}=\left[\begin{array}{lll}
R_{1}^{b}(1,3) v_{n}-R_{1}^{b}(1,2) v_{u} & R_{1}^{b}(1,1) v_{u}-R_{l}^{b}(1,3) v_{e} & R_{1}^{b}(1,2) v_{e}-R_{1}^{b}(1,1) v_{n} \\
R_{1}^{b}(3,3) v_{n}-R_{1}^{b}(3,2) v_{u} & R_{1}^{b}(3,1) v_{u}-R_{1}^{b}(3,3) v_{e} & R_{1}^{b}(3,2) v_{e}-R_{l}^{b}(3,1) v_{n}
\end{array}\right]
\end{aligned}
$$

and $\mathbf{R}_{\mathbf{l}}^{\mathbf{b}}(\mathbf{i}, \mathbf{j})$ is the element in the ith row and $j$ th column of $\mathbf{R}_{\mathbf{l}}^{\mathbf{b}}$.

More details can be found in [33]. 


\section{Chapter 4}

\section{Hardware and Software Implementation}

\subsection{Hardware Implementation}

\subsubsection{Inertial Sensor Output Acquisition}

For the low-cost objective, the IMU on the navigation board consists of a reduced set of inertial sensors involving one single-axis $\pm 80^{\circ}$ /sec yaw rate gyroscope ADIS16060 [34] and one dual-axis $\pm 1.7 \mathrm{~g}$ accelerometer ADIS16003 [35] from Analog Devices Inc. This accelerometer and gyroscope are operated on a voltage supply of $3.3 \mathrm{~V}$ and give 2048 and 800 digital outputs as their zero-g and zero-rate respectively. The sensitivity of the accelerometer and gyroscope is typically 0.0122 \%sec/LSB and $820 \mathrm{LSB} / \mathrm{g}$, respectively. The specifications are given in Chapter 5.

\subsubsection{GPS Data Acquisition}

GPS receiver LEA-5H from $\mu$-blox is used in the proposed integrated system. Its compact size, low power consumption, highly reliable positioning performance $(2.5 \mathrm{~m} \mathrm{CEP})$ and its SuperSense feature (high sensitivity and High jamming immunity, refer to [36] for more details) with 18 tracking channels make it an outstanding choice amongst off-the-shelf commercial products.

GPS transmits data using the UART interface and it uses National Marine Electronics Association (NMEA) data communication protocol to transmit positional information. The serial data, in the form of NMEA sentences, are transmitted in ASCII code. The data acquisition process is performed on DSP, which parses the NMEA sentences, extracts useful information such as latitude, longitude and time tag, and then converts them into binary code. 


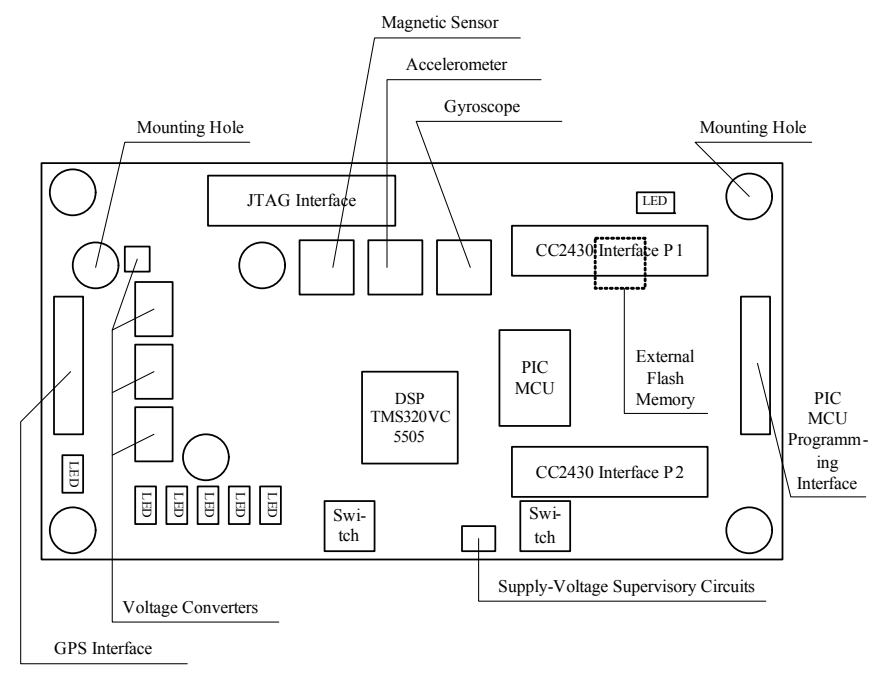

Figure 4.1 Layout of the navigation board.

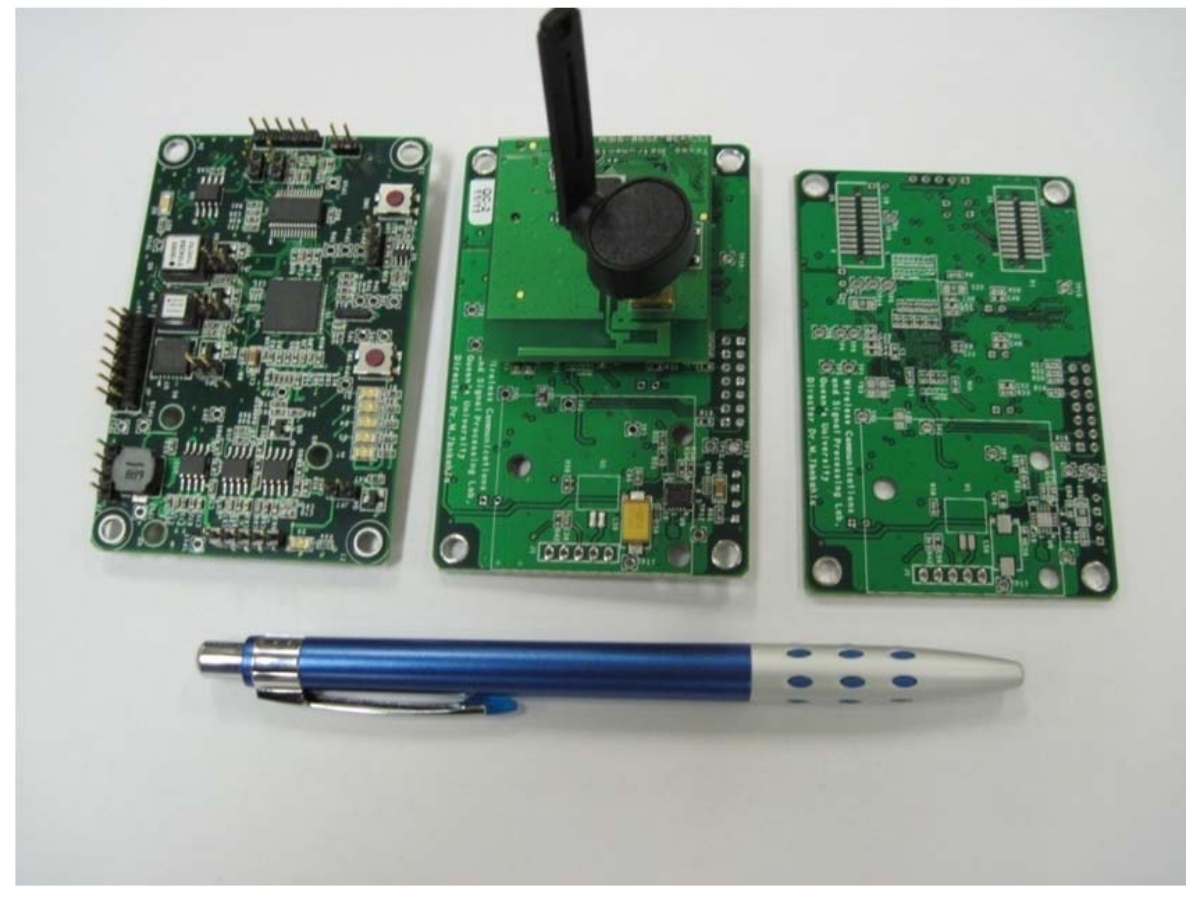

Figure 4.2 PCB layout of the proposed system.

\subsubsection{Navigation Data Processing}


The DSP used for this design is a 196 pin fixed-point TMS320VC5505, which is a member of TI's TMS320C5000 family. This DSP is well designed for low-power applications, capable of operating at a maximum CPU frequency of $100 \mathrm{MHz}$ when the core voltage is $1.3 \mathrm{~V}$.

The DSP clock, which is used by the CPU and most of the peripherals, is controlled by the system clock generator. The clock generator can either be driven by the $32.768-\mathrm{KHz}$ on-chip realtime clock (RTC) oscillator or accept an input reference clock from the CLKIN pin. In the present design, the $32.768-\mathrm{KHz}$ internal oscillator is used to provide the clock signals to both the RTC timer and the DSP clock generator by setting the CLK_SEL pin low [37]. To generate a system clock signal as high as $100 \mathrm{MHz}$, the phase-locked loop (PLL) circuit in the clock generator is programmed to multiply the input clock $32.768 \mathrm{KHz}$, by using a 3052x multiplier rate, which is controlled through the PLL control registers.

To improve the real-time performance and reduce the data acquisition overheads, FPGA is used in many GPS/INS applications to assist DSP in receiving navigation data. The integration of a FPGA and a DSP can significantly improve the calculation efficiency since DSP can directly fetch the data instead of waiting for the low speed serial I/O operation. Although the real-time system may benefit from FPGA in high sampling data-rate cases, a single-chip DSP can provide comparable performance when the sampling data rate is low. And most importantly, a single DSP chip makes the navigation system simpler and more compact, which meets the requirements of energy-sensitive applications and cost-sensitive applications.

\subsubsection{RF Transceiver}


CC2430 is a System-on-Chip solution from TI that contains a high performance and low power 8051 processor core and 2.4 GHz IEEE 802.15 .4 compliant RF transceiver. It is highly suited for systems that require ultra-low power consumptions. When the microcontroller is running at $32 \mathrm{MHz}$, the current consumption of the transmitter/receiver is typically as low as $27 \mathrm{~mA}$. In addition, it features by four flexible power modes with very short transaction time between low-power modes and active mode, which can effectively reduce the average power consumption in low duty cycle systems.

To measure the communication range, a range test has been conducted in an open field environment, with one transmitter placed on the ground and one receiver placed one meter above the ground. In this test, the transmitter continued to send data packets to the receiver. CC2430 has a builtin Received Signal Strength Indicator (RSSI) giving a digital value that can be read from one of its special function registers. The RSSI used in this test was the average RSSI of the last 32 received packets. The Packet Error Rate (PER) and RSSI are the parameters that are used to determine the communication quality of this test.

When PER was not increasing, and RSSI was higher than $-75 \mathrm{dBm}$, the communication quality was considered acceptable. Otherwise, the received signal was regarded as weak and unstable.

Based on the range test, the effective range of the radio link between two CC2430 nodes is approximately 277 meters, when the transmit power is $19 \mathrm{dBm}$ (with a device current consumption of 32.4 $\mathrm{mA}[38]$ ), and the wireless transmission is a LOS propagation.

\subsubsection{Power Supply of the Navigation Board}


The power supply set required for the navigation board is $[1.3 \mathrm{~V}, 1.8 \mathrm{~V}, 3.3 \mathrm{~V}, 5 \mathrm{~V}]$. The $3.3 \mathrm{~V}$ and $5 \mathrm{~V}$ are used by all the peripherals. DSP requires $1.3 \mathrm{~V}$ and $1.8 \mathrm{~V}$ for core power and $3.3 \mathrm{~V}$ for the $\mathrm{I} / \mathrm{O}$ pins. Three "AA" size batteries $(3 \times 1.5 \mathrm{~V})$ are used to provide the DC power, which is initially regulated at $5 \mathrm{~V}$ by a fixed-output boost converter TPS61032 [39]. This converter also provides an interrupt signal (INT0) required by DSP. Then $1.3 \mathrm{~V}, 1.8 \mathrm{~V}$ and $3.3 \mathrm{~V}$ power supplies are generated by using three adjustable low-dropout voltage regulators TPS76601 [40]. The DSP power-up sequence specifically requires that the core-level supplies $(1.3 \mathrm{~V}$ and $1.8 \mathrm{~V})$ must power up before the $\mathrm{I} / \mathrm{O}$ level supplies $(3.3 \mathrm{~V})$. Therefore, the ENABLE signal of the $5 \mathrm{~V}$-to-3.3V converter is connected with the POWERGOOD pin of the $5 \mathrm{~V}$-to-1.3V converter through a NPN transistor to guarantee a sufficient time delay between the core power-up and I/O power-up. More details of the power supply circuit design can be found in Figure A.4, Appendix A.

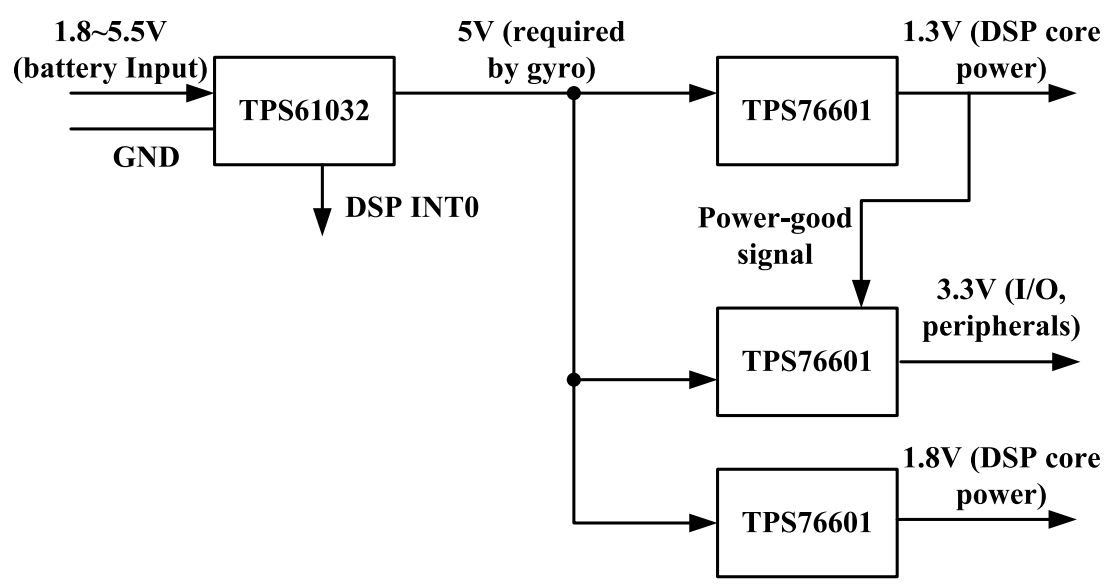

Figure 4.3 Design of the power circuit.

\subsubsection{Connectors/Jumpers on Navigation Board}


As shown in Figure 4.1, the navigation board has two 20-pin SMD connection headers for interfacing with the CC2430EM board. This board is also supplied with a standard 14-pin JTAG header interface that is used by emulators to interface with DSP and a 5-pin header that is used as the programming interface of PIC16F886 MCU. There is a 2-pin jumper in parallel with a Bipolar Junction Transistor (BJT) that is connected with the power supply of each power driven component (i.e. GPS, inertial sensors, and magnetic sensor) and controlled by the PIC16F886 MCU. These bipolar junction transistors are used as power-on/off switches and controlled by software. This power management ability will be disabled when the 2-pin jumpers are connected.

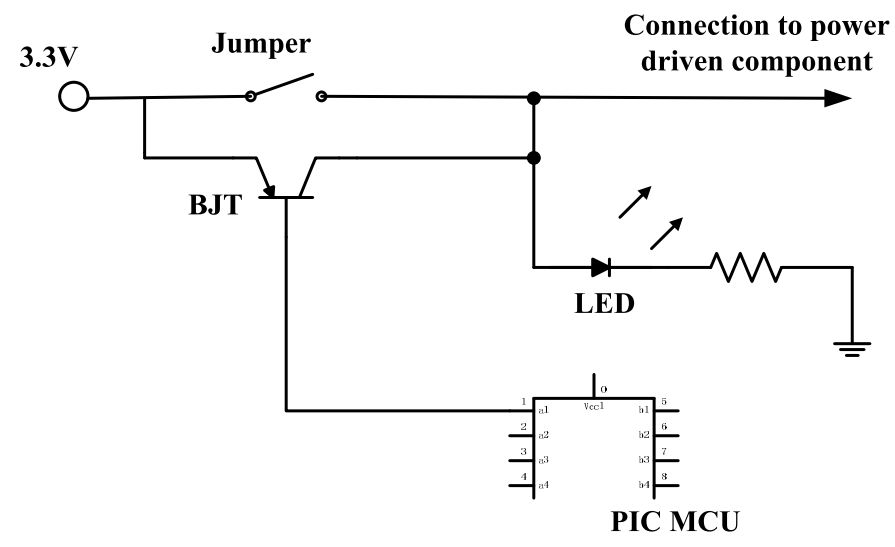

Figure 4.4 Power control design with a 2-pin jumper and a bipolar junction transistor.

\subsubsection{Reset and Wakeup Circuits}

The reset circuit of DSP is based on the supply-voltage supervisory circuits TPS3106K33 [41] and a push button switch. When the power reset signal is selected as the source of the DSP reset signal and the power supply is less than the threshold voltage $2.941 \mathrm{~V}$, TPS3106K33 will generate a reset signal 
to the DSP. The reset source is selected by a three position jumper and Table 4.1 below shows the positions and the corresponding functions:

Table 4.1 Reset source select of the three position jumper.

\begin{tabular}{|c|c||}
\hline Jumper Position & Function \\
\hline \hline $1-2$ & The power reset signal is the source of the DSP reset signal. \\
\hline $2-3$ & The push button switch is the source of the DSP reset signal. \\
\hline
\end{tabular}

\subsection{Development Tools}

\subsubsection{IAR Embedded Workbench for 8051}

IAR Embedded Workbench is a development environment that includes a $\mathrm{C} / \mathrm{C}++$ compiler and debugger and provides extensive support for a wide range of 8051 devices. The software development of CC2430 is supported by the IAR EW8051.

\subsubsection{Code Composer Studio}

Code Composer Studio (CCS) is the Integrated Development Environment (IDE) for high-level TI C and assembly language that includes compilers for each of TI's device families, a source code editor, a project build environment, a debugger, a profiler, simulators, etc. It directly runs on the target DSP by gaining access to special function registers, internal memory, and peripherals in order to build source codes, as well as run and debug applications.

\subsubsection{CCS Simulator}


CCS simulator is used to simulate the TMS320C55x microprocessor for software development and program verification in non-real time. It can debug the programs without target hardware. The simulator uses the standard $\mathrm{C}$ or assembly source debugger interface, allowing the user to debug the programs in $\mathrm{C}$ or in assembly language or both.

The codes of the EKF algorithms were written in C language, which were then converted into assembly source codes by the compiler. After the assembly source codes were generated, the assembler produced an assembly listing with offsets, which was stored in an object file in the Common Object File Format (COFF). Afterwards, the object file was input to the linker to produce an executable file.

The DSP real-time performance of the GPS/INS data reading and navigation computation was analyzed by the CCS simulator, which will be discussed in Section 5.2.

\subsubsection{CCS Emulator}

Once the codes are debugged and run on the simulator, they are downloaded on the target processor using an emulator through JTAG interface. An emulator is a powerful, high-speed hardware development system that emulates hardware operations on target DSP. Access to DSP registers and on-chip memories are available to help users easily debug programs running on target hardware. The emulator selected for DSP TMS320VC5505 in the navigation board is TI XDS100v2.

\subsection{System Flow}

\subsubsection{Program Flow of System Initialization}


The system initialization routine includes DSP clock frequency configuration, parallel port configuration, and the initialization of its peripherals, which is shown in Figure 4.5. The DSP interrupt vector table is defined in assembly codes, which is described in Table 4.2.

Table 4.2 Definitions for interrupt vectors.

\begin{tabular}{|c||c|c|c||}
\hline Name & ISR & Priority & Function \\
\hline \hline RST & Reset_ISR & 0 & Hardware/Software Reset Interrupt \\
\hline \hline INT0 & BattPower_ISR & 3 & External User Interrupt \#0 \\
\hline \hline UART & UART_ISR & 9 & UART Receive Interrupt \\
\hline \hline RTC & Wakeup_ISR & 12 & Wakeup or real-time clock interrupt \\
\hline
\end{tabular}

Various external interrupts and ISRs are described as follows:

- As shown in Figure A.2, whenever a reset signal is generated at pin D6 of C5505, the DSP terminates execution and loads the program counter with the contents of the reset vector, which leads the program to go back to the on-chip ROM bootloader. After completing the reset ISR, the program restarts the initialization function.

- When the battery voltage is lower than $1.8 \mathrm{~V}$ (making the voltage at LBI lower than $500 \mathrm{mV}$ ), the low-power detection circuit shown in Appendix A causes the LBO pin to generate a logic low signal that forces the program to jump into the BattePower_ISR. This ISR stops the program executions and flashes the LED on pin M8 as a low-battery-power warning.

- As shown in Figure 4.6, when up to 70 bytes of serial data (approximately as long as a GPRMC sentence with full information) are sent from the GPS to the DSP through UART, this UART 
interrupt event will occur. The location and time information will then be abstracted from the raw serial data and saved if the GPS receiver has enough visible satellites.

- DSP enters sleep mode when the navigation board is stationary. When motion is detected by PIC

MCU, a logic-high signal will be sent to wake up the DSP core from idle condition. More details are described in section 4.3.2.2. The wake-up signal can also be generated by a push button on the PCB. The wake-up ISR is shown in Figure 4.8.

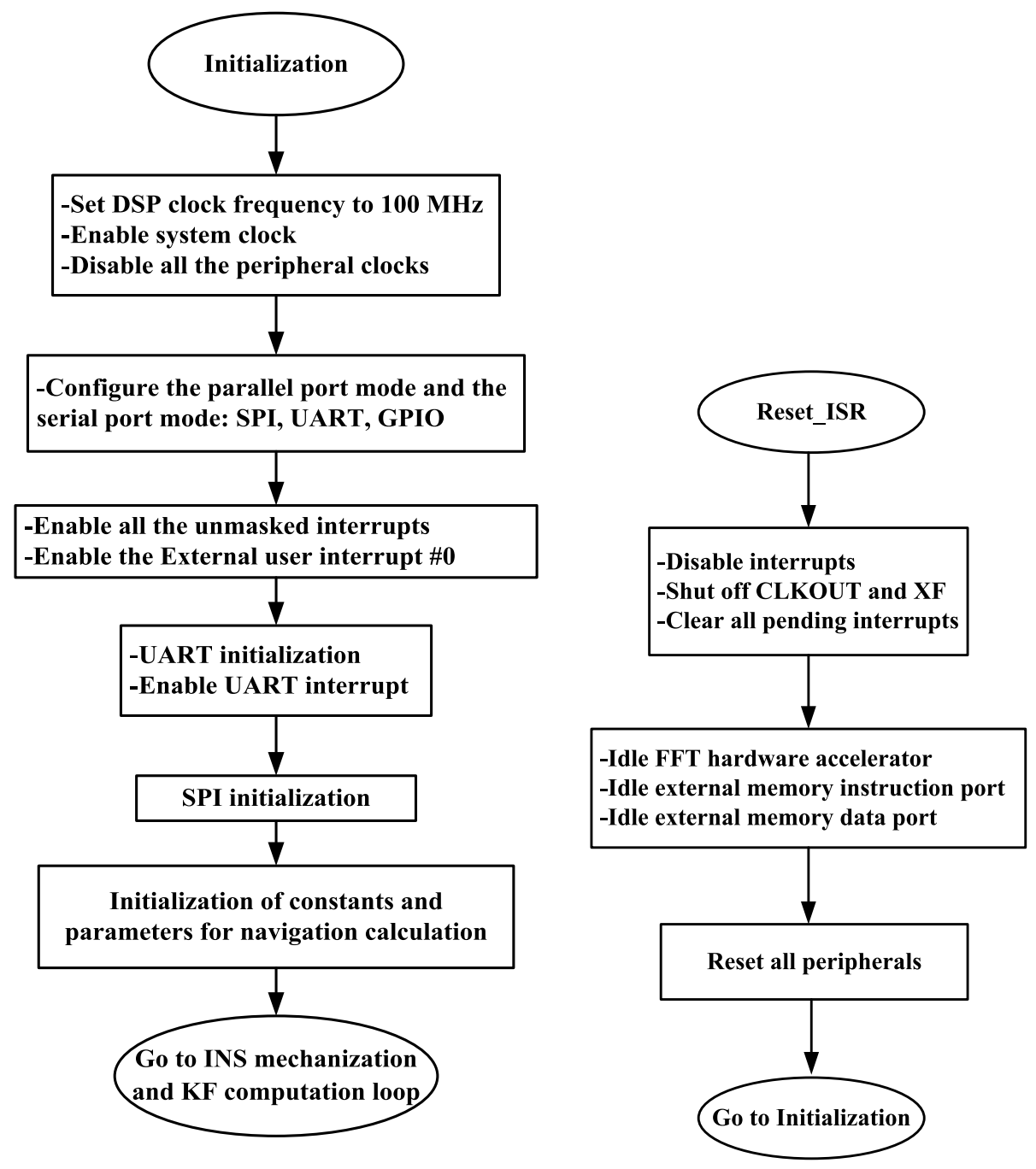

Figure 4.5 DSP initialization routine and DSP reset ISR. 


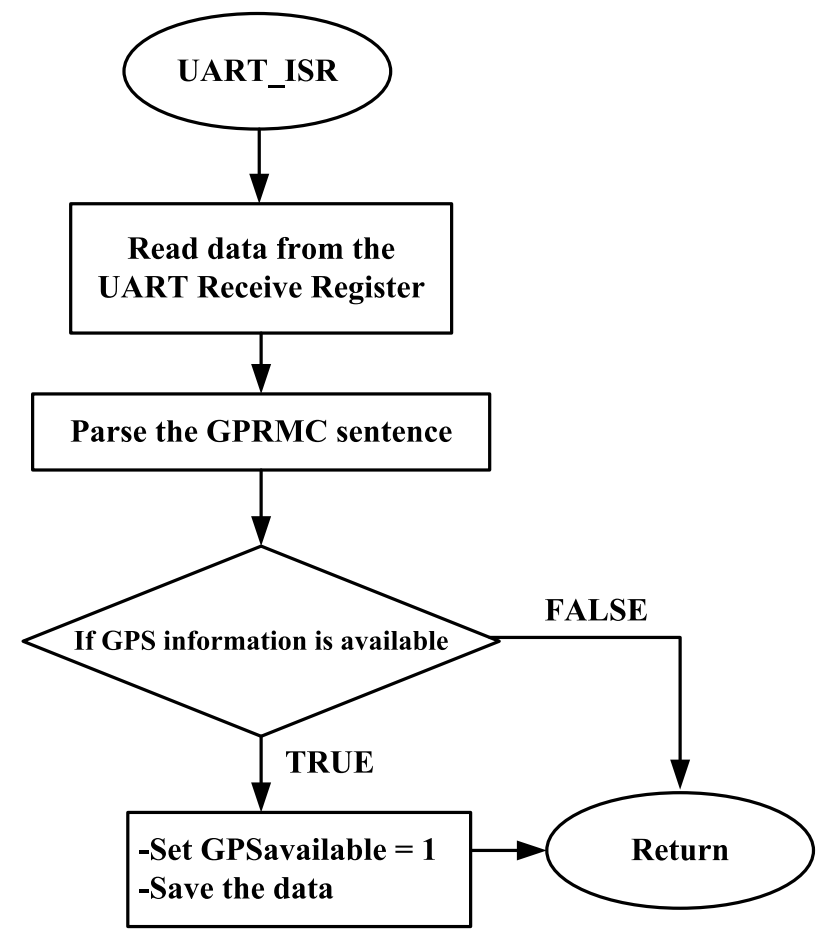

Figure 4.6 Flow chart for UART receive ISR.

\subsubsection{Power Management Scheme}

\subsubsection{Ultra Low-Power Motion Detection}

Power consumption is usually one of the dominating design criteria in navigation system development. To minimize the system power, a motion detection scheme is proposed in this thesis to optimize the power management design. When the navigation board is stationary and its position is fixed, both GPS and inertial sensors will be turned off until it starts to move again.

PIC16F886 MCU is used to accomplish the motion detection design, which is based on an external-interrupt trigger built on the PCB. When the object (a pedestrian, an animal, or a vehicle, etc.) moves or shakes, the trigger mounted on this object will detect its vibration and send an external interrupt to the PIC MCU. This MCU is featured with an ultra-low-power wake-up function that 
provides an efficient technique by periodically waking up the chip from sleep. It charges a capacitor, enables a low voltage interrupt, and goes to sleep. When this capacitor slowly discharges below $\mathrm{V}_{\text {IL }}$, a wake-up interrupt will wake up the controller again. The design idea of the motion detection scheme is to keep the PIC MCU alternately going to sleep and waking up to maximize the sleep time of the microcontroller so that the overall power consumption is minimized. Figure 4.7 shows the flow chart of the motion detection scheme. The microcontroller waits for the external interrupt for a certain period (i.e. detection period is set by a timer). If there is no external interrupt during the detection period, a timer interrupt will be generated and the microcontroller will go into ultra-low-power sleep mode. Otherwise, if the target shakes or its movement is detected, a counter will increase and the MCU will go into the motion reconfirming mode and wait for the next external interrupt. If the counter reaches the motion threshold, then MCU will send a message to DSP indicating that a constant motion is detected.

\subsubsection{Power Management of C5505 and CC2430}

In many applications, in wireless sensor networks, for instance, there are specific requirements to minimize power consumption and prolong the lifetime of the embedded system with battery power supply. C5505 has several means of managing the power consumption, which is detailed as follows:

- C5505 can selectively activate some subsystems while keeping other subsystems inactive by using software-controlled module clock gating;

- When not operating, the on-chip memory can be placed in a low-leakage-power mode while preserving the memory contents (DARAM/SARAM low-power modes). 


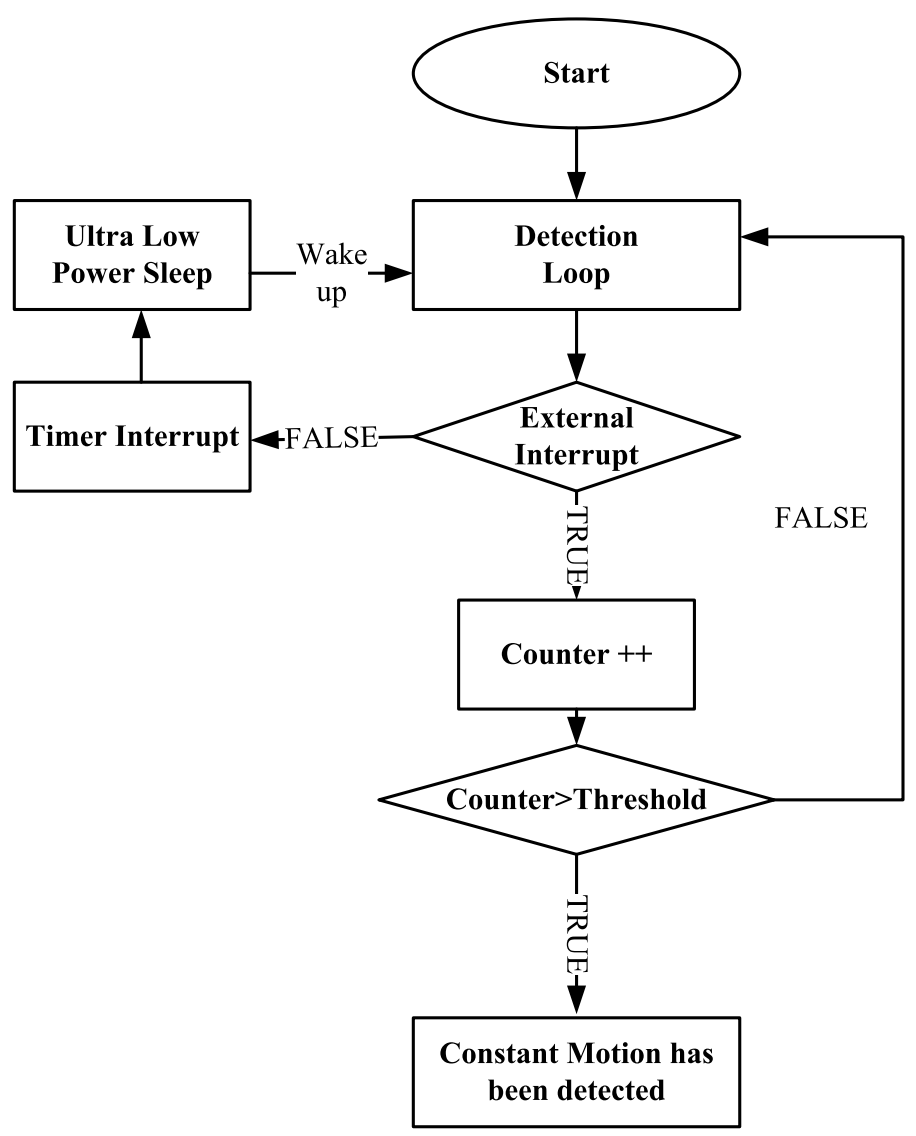

Figure 4.7 flow chart for motion detection.

- Independent power domains allow users to power down parts of the DSP to reduce static power consumption.

Since the navigation board uses batteries as its power supply, low-power consumption is a primary requirement for software design. According to the signal sent from the motion detection MCU, DSP operates in either sleep mode or active mode and switches from one to the other alternatively to save power. The power modes are described as:

\section{Sleep Mode}

- system clock is disabled, all the peripheral clocks are disabled; 
- on-chip memory in memory retention mode (placed in a low-leakage-power mode while preserving memory contents)

- disable the clock generator domain by placing the system clock generator in bypass mode and putting the PLL in power down mode;

- clear and disable all interrupts;

- enable the appropriate wake-up interrupt.

\section{Active Mode}

- system clock and I2C, UART, SPI clocks are enabled;

- on-chip memory in active mode;

- enable the clock generator domain;

- periodically check the GPIO pin of motion detection MCU. If the output goes low, make DSP go to sleep mode again.

The subprogram flows of DSP_Sleep function and the Wake-up ISR are given below in Figure 4.8 .

Similarly, CC2430 operates in its full functional mode PM0 (more information is provided in [16]) when the motion detection MCU indicates the board is moving. If the board is stationary for a certain time, CC2430 will enable an external IO interrupt and enter PM3 with the lowest power consumption, in which all internal circuits that are powered from the voltage regulator are turned off. Once the board starts to move again, CC2430 will be woken up by the enabled external interrupt event sent from PIC MCU, enter PM0 and start from where it entered PM3 before. 

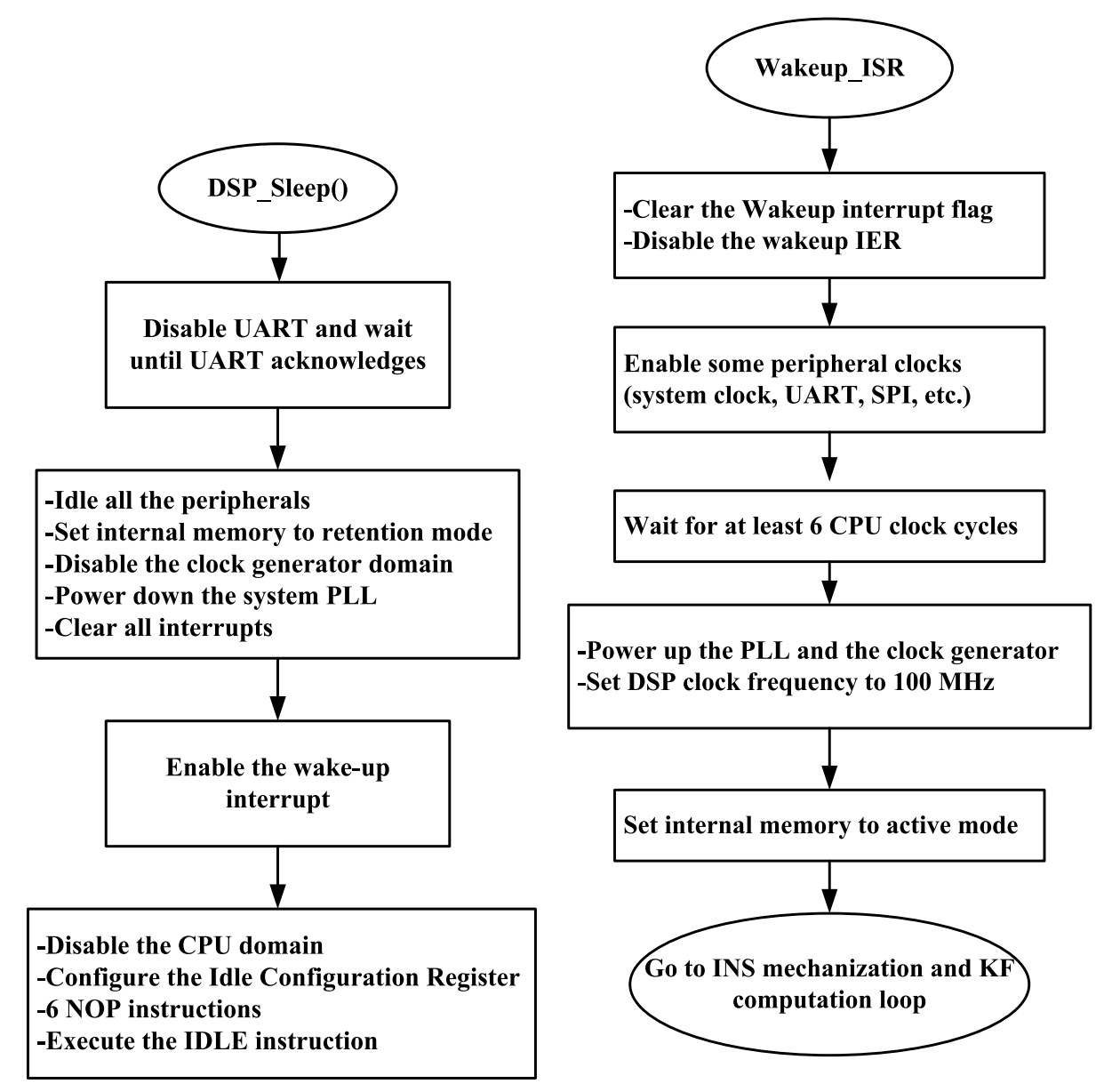

Figure 4.8 Program flows of DSP_Sleep and Wakeup_ISR. 


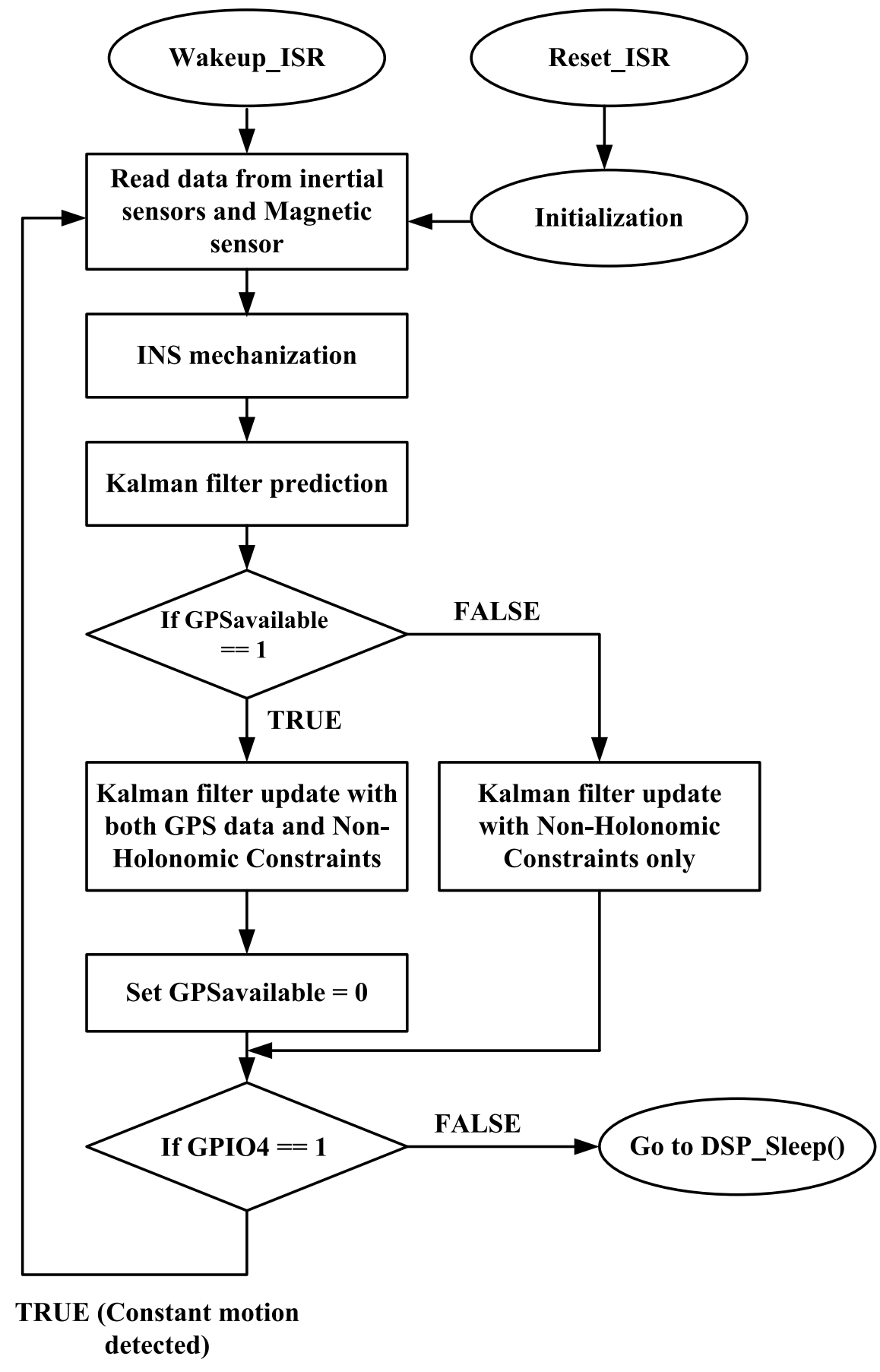

Figure 4.9 Software flow of the whole system.

\subsubsection{DSP Memory Allocation}


The navigation computation of C5505 has been tested by running the DSP in a CCS emulator environment. Most of the variables involved in the computation are represented in the double precision format (64 bits). Since C5505 is a fixed-point DSP and the quantization errors yielded by truncating/rounding numbers during intensive computation cannot be overlooked, the DSP computational accuracy cannot be compared to output provided by computer software simulations (Such as Matlab). By inputting the same data and comparing the outputs from DSP and Matlab, the differences between the DSP results and the Matlab results are shown in Table 4.3.

Table 4.3 DSP computation accuracy compared with matlab.

\begin{tabular}{|c|c|c|c|c|c|}
\hline & Latitude & Longitude & $\begin{array}{c}\text { North } \\
\text { velocity }\end{array}$ & $\begin{array}{c}\text { East } \\
\text { velocity }\end{array}$ & Yaw \\
\hline \multirow{3}{*}{ Differences } & $<0.000002^{\circ}$ & $<0.000002^{\circ}$ & $<0.4 \mathrm{~m} / \mathrm{s}$ & $<0.4 \mathrm{~m} / \mathrm{s}$ & $\begin{array}{c}<0.020 \mathrm{rad} / \mathrm{sec} \\
(1.1459 \% \mathrm{sec})\end{array}$ \\
& $(0.21577 \mathrm{~m})$ & $(0.21577 \mathrm{~m})$ & & & \\
\hline
\end{tabular}

The C5505 has three types of on-chip memory: 128 KB Read-Only Memory (ROM), 256 KB Single-Access Random Access Memory (SARAM) and 64 KB Dual-Access Random Access Memory (DARAM). As the internal memory is quite sufficient for the navigation computation algorithms and system operations, the DSP requires no external interface of RAM. The memory allocation map generated by CCS is shown in Table 4.4. From this table, we can see that the total memory usage is $86.7481 \mathrm{~K}$ bytes and $46.3848 \mathrm{~K}$ bytes are occupied in DARAM, including 35000 bytes of heap, 5000 bytes of stack, and 5000 bytes of system stack. 
Table 4.4 Memory map report generated from CCS.

\begin{tabular}{|c|c|c|c|c|}
\hline Name & Origin (bytes) & Length (bytes) & Used (bytes, Hex) & Used (bytes, Dec) \\
\hline MMR & 00000000 & $000000 \mathrm{c} 0$ & 00000000 & 0 \\
\hline DARAM & $000000 \mathrm{c} 0$ & 0000ff40 & 0000b98a & $46.3848 \mathrm{~K}$ \\
\hline SARAM & 00030000 & $0001 \mathrm{e} 000$ & $0000 \mathrm{a} 174$ & $40.3633 \mathrm{~K}$ \\
\hline SAROM_0 & $00 \mathrm{fe} 0000$ & 00008000 & 00000000 & 0 \\
\hline SAROM_1 & $00 \mathrm{fe} 8000$ & 00008000 & 00000000 & 0 \\
\hline SAROM_2 & 00ff0000 & 00008000 & 00000000 & 0 \\
\hline SAROM_3 & $00 \mathrm{ff} 8000$ & 00008000 & 00000000 & 0 \\
\hline \multicolumn{4}{|c|}{ Bytes occupied in total } & $86.7481 \mathrm{~K}$ \\
\hline
\end{tabular}

\subsubsection{Zigbee Node Software Design}

A typical Zigbee sensor network is composed of three logical device types, i.e. coordinator, router and end device. This section presents the software flow of sensor nodes, routers and sink node in Zigbee networks. The sink node works as the coordinator, while sensor nodes work as the end devices, which are shown in Figure 4.10.

\section{- Sink Node}

SmartRF04EB with CC2430EM from TI [43] is used to develop and debug the sink node programs in this work. It will initialize the board hardware configurations, create a network identifier, and broadcast PAN ID to start up the network. When the power is turned on, this coordinator will scan all channels and choose the one with the least energy level. If there are more than one channel with low energy level, it will choose the channel with the least existing ZigBee networks. 


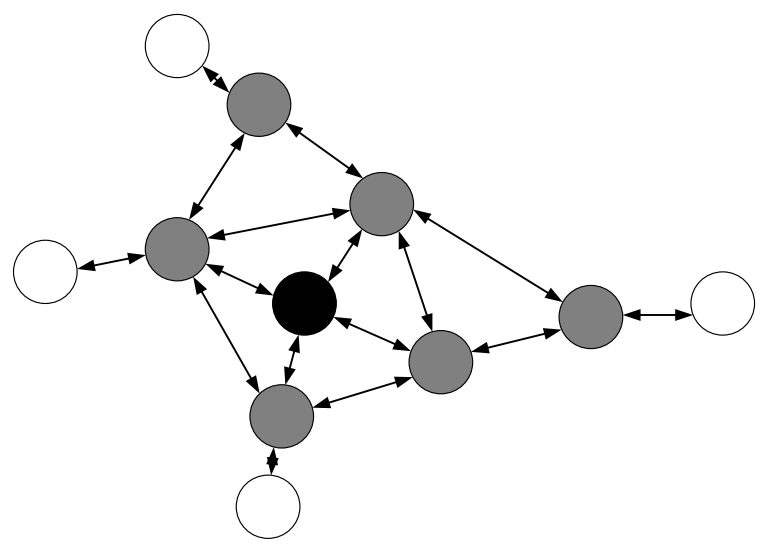

Figure 4.10 The structure of a typical ZigBee sensor network with the coordinator (in black), the routers (in grey) and the end devices (in white).

The task system is based on the multitask mechanism, and its main loop operates in the Operating System Abstraction Layer (OSAL) which implements a cooperative, round robin task servicing loop. Each OSAL task has to be initialized first, including initialization of application object variables, instantiation of the corresponding application object(s), and registration with the applicable OSAL or Hardware Abstraction Layer (HAL) system services. User defined events will be added into the task event processor to process all events for the task. The initialization and configuration of the sink node program flow is shown in Figure 4.11 and the event processing flow of the sink node task event handler is shown in Figure 4.12. 


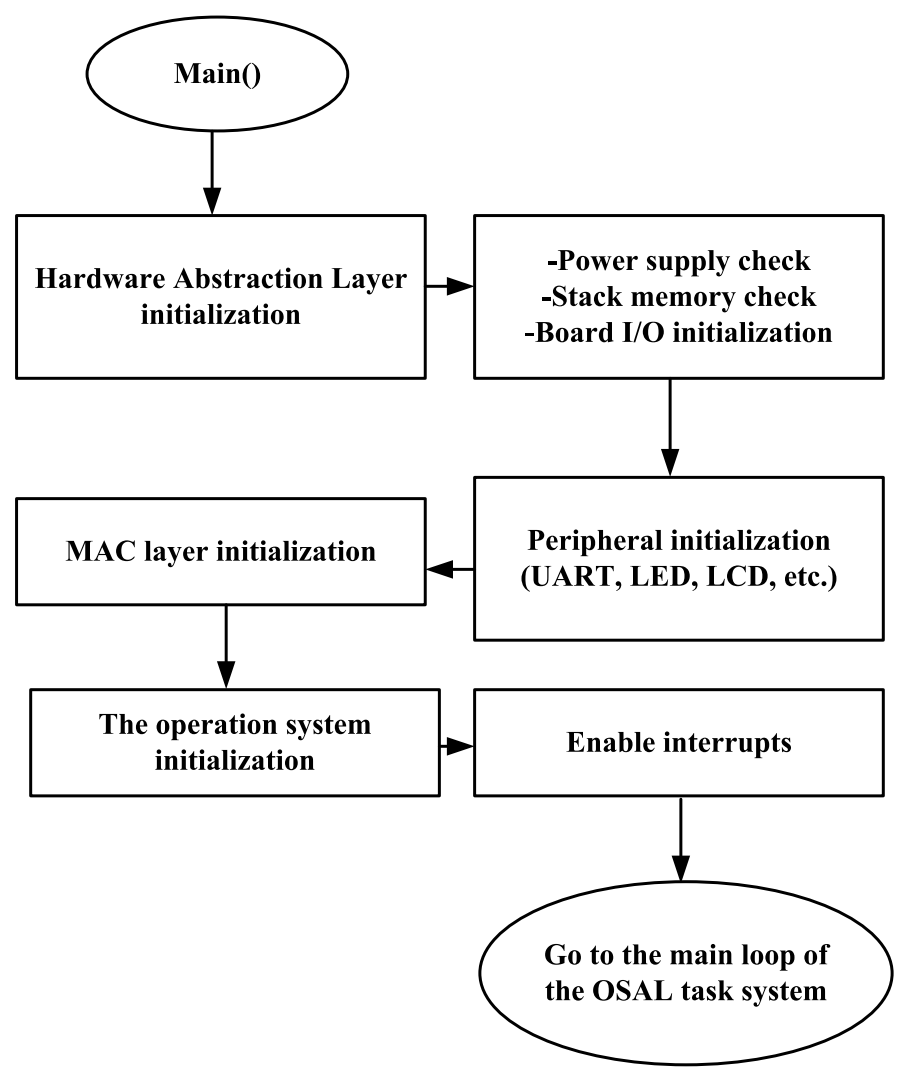

Figure 4.11 The initialization and configuration of the sink node program flow.

- Router

This device is used to allow other devices to join the network and route messages from one node to another. The routers, as well as end devices, scan the channels to find and join the available network with the identifier specified by the coordinator. CC2430 demonstration boards (CC2430DB) are used as the routers to compose the ZigBee networks. 


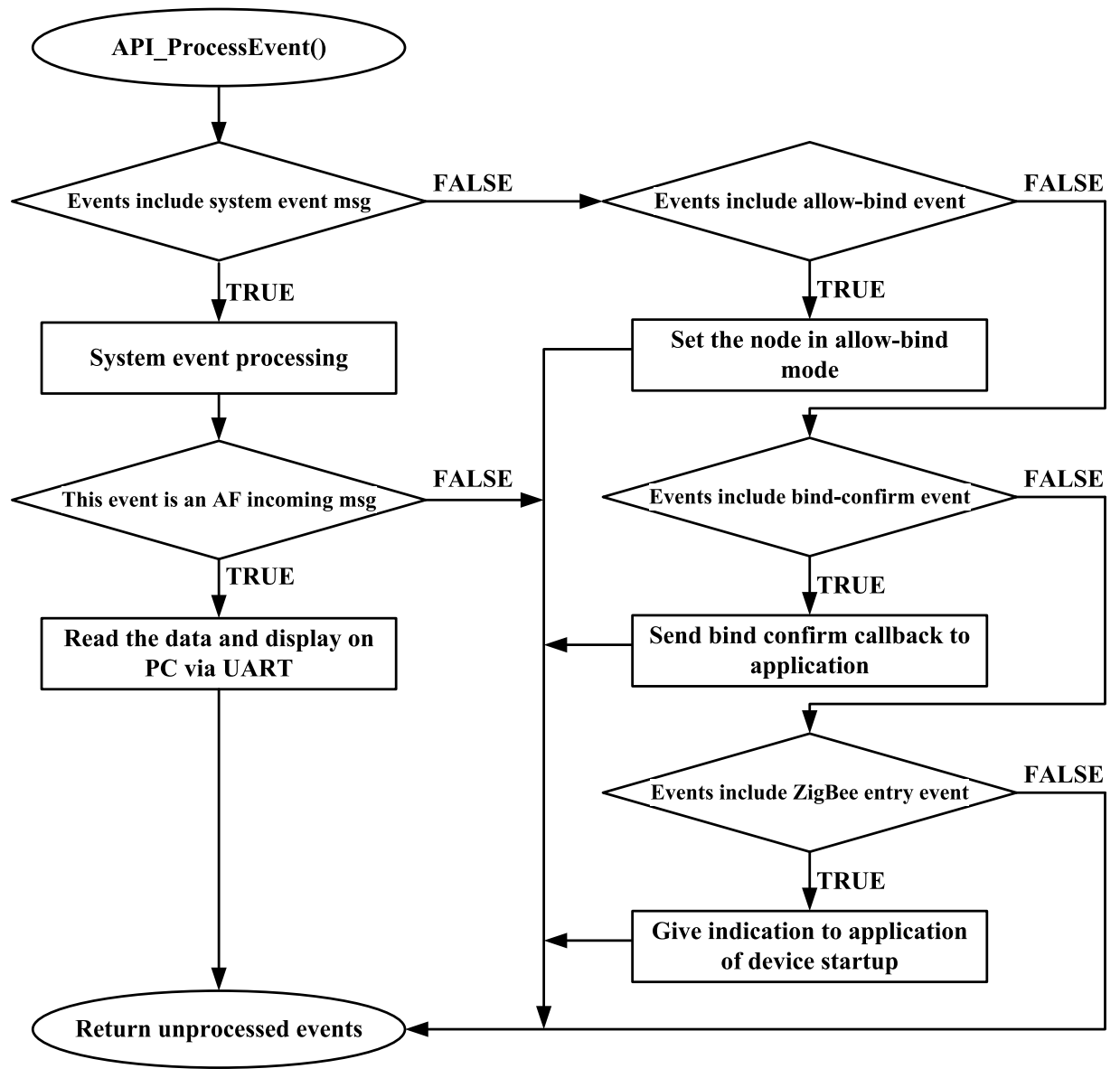

Figure 4.12 The event processing flow of the sink node task event handler.

\section{- Sensor Node}

The sensor node works as an end device in the ZigBee network. This device can only receive and transmit information to the parent node (routers or the coordinator) and it has no routing capability.Therefore, it is a battery-powered node and can sleep and wake up according to the network requirements. The navigation board with a CC2430 RF transceiver is used to work as an end device in this network. The initialization and configuration of the sensor node program flow is the same as Figure 4.11 and the event processing flow of its task event handler is shown in Figure 4.13. 


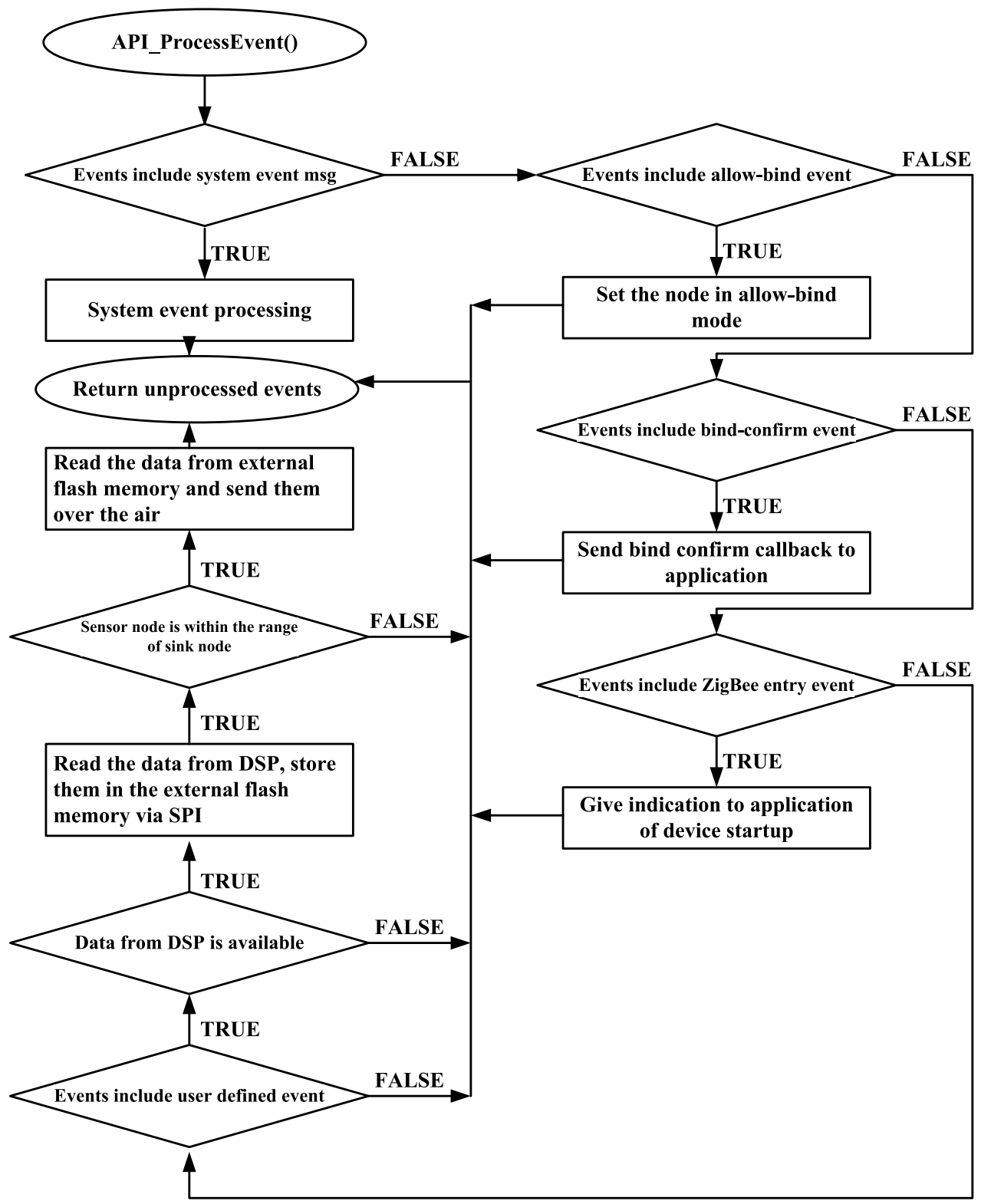

Figure 4.13 The event processing flow of the sensor node task event handler. 


\section{Chapter 5}

\section{Tests and Results}

\subsection{Equipment and Setup}

The MEMS inertial sensors being used in the laboratory calibration and field test II are the three-axis $\pm 3 \mathrm{~g}$ accelerometer ADXL335 [44] from Analog Devices Inc. and the single-axis $\pm 300 \%$ s yaw rate gyroscope LISY300AL [45] from STMicroelectronics. These accelerometer and gyroscopes are operated on a voltage supply $3.3 \mathrm{~V}$ and give $1.5 \mathrm{~V}$ and $1.65 \mathrm{~V}$ respectively as their zero-g and zero-rate voltage level. The sensitivity of the accelerometer and gyroscope is typically $300 \mathrm{mV} / \mathrm{g}$ and 3.3 $\mathrm{mV} / \% / \mathrm{s}$, respectively. The specifications for these analog inertial sensors are shown in Table 5.1. The analog signals from accelerometers and gyroscopes are sampled by a multi-channel ADC which processes the inertial sensor measurements and converts them into digital signals. A 14-bit, $285 \mathrm{KSPS}$ ADC from Analog Devices Inc. (AD7856) [46] is chosen for sampling the inertial sensor measurements. A multiplexer is used for multi-channel sampling at the input stage of ADC. Therefore, a time delay $(\Delta \mathrm{t})$ exists between the sampling of two sequential channels [15]. Since the conversion time $(\Delta \mathrm{t})$ is sufficiently short depending on the master clock signal (max. $6 \mathrm{MHz})$ for $\mathrm{ADC}$, the effect of errors due to phase delays is negligible. The specifications for the inertial sensors and ADC are given in Table 5.1. The GPS receiver used in the tests is LEA-5H from $\mu$-blox, which was introduced in Chapter 4. 


\subsection{Real-Time Performance Analysis}

The DSP real-time performance was evaluated by CCS C55xx Rev 3.0 Cycle Accurate Simulator. Each assembly instruction is executed with a certain number of instruction cycles, and the duration of each mechanization computation and Kalman filtering computation can be calculated by counting the number of cycles the program takes.

Table 5.1 Specifications for ADXL335 and LISY300AL.

\begin{tabular}{|c|c|}
\hline Parameter & Typical Value \\
\hline \multicolumn{2}{|c|}{ Gyroscope LISY300AL } \\
\hline Measurement range & $\pm 300 \% / \mathrm{s}$ \\
\hline Sensitivity & $3.3 \mathrm{mV} / \% / \mathrm{s}$ \\
\hline Zero-rate level & $1.65 \mathrm{~V}$ \\
\hline Non linearity & $\pm 0.8 \%$ of full scale \\
\hline Noise density & $0.1 \% / \mathrm{s} / \sqrt{\mathrm{Hz}}$ \\
\hline \multicolumn{2}{|c|}{ Accelerometer ADXL335 } \\
\hline Measurement range & $\pm 3.6 \mathrm{~g}$ \\
\hline Sensitivity & $300 \mathrm{mV} / \mathrm{g}$ \\
\hline Zero-rate level & $1.5 \mathrm{~V}$ \\
\hline $\begin{array}{l}\text { Non linearity } \\
\end{array}$ & $\pm 0.3 \%$ of full scale \\
\hline Noise density at Xout, Yout & $150 \mu \mathrm{g} / \sqrt{\mathrm{Hz}} \mathrm{RMS}$ \\
\hline Noise density at Zout & $300 \mu \mathrm{g} / \sqrt{\mathrm{Hz}} \mathrm{RMS}$ \\
\hline
\end{tabular}

As shown in Table 5.2, the number of instruction cycles required for one mechanization computation is 84709 ; one Kalman filtering computation requires 4023352 cycles. When the DSP runs at $100 \mathrm{MHz}$, the duration of each instruction cycle is $10 \mathrm{~ns}$. Therefore, the execution time for each step of mechanization computation takes $0.8 \mathrm{~ms}$ while each step of Kalman filtering computation takes $40 \mathrm{~ms}$. Based on these results, the duration of GPS and INS data reading can be calculated by running the prototype navigation board, which is introduced in Section 5.1, recording the time and using the CCS emulator to count how many times the navigation calculation loop repeats 
in this period. The duration of INS data reading for each time was approximately $4.65 \mathrm{~ms}$ on average, and the program took nearly $90.909 \mathrm{~ms}$ to read and parse the GPS data whenever it was updated (when GPS is available, it will update every second). According to the software design introduced in Chapter 4, the navigation calculation is running in an infinite loop. In each step, INS reading, mechanization and Kalman filtering computation will be executed successively. In contrast, GPS data reading runs in an interrupt service routine. Therefore, when a GPS update is available, DSP with 100 $\mathrm{MHz}$ clock rate can run the navigation calculation loop 20 times and get 20 position results (as well as the velocity and heading information) per second; otherwise, if there is no GPS update, DSP can get 22 position results per second. Figure 5.1 illustrates the DSP computation time frame.

Table 5.2 Execution time for the Program.

\begin{tabular}{|c|c|c|}
\hline & Number of CPU Cycles & $\begin{array}{c}\text { Execution Time on DSP } \\
\text { operated with 100 MHz }\end{array}$ \\
\hline Initialization & & $<2 \mathrm{~ms}$ \\
\hline GPS Data Reading & & $90.909 \mathrm{~ms}$ (per second) \\
\hline INS Data Reading & & $4.65 \mathrm{~ms}$ \\
\hline Mechanization & 84709 & $0.8 \mathrm{~ms}$ \\
\hline Kalman Filter & 4023352 & $40 \mathrm{~ms}$ \\
\hline
\end{tabular}

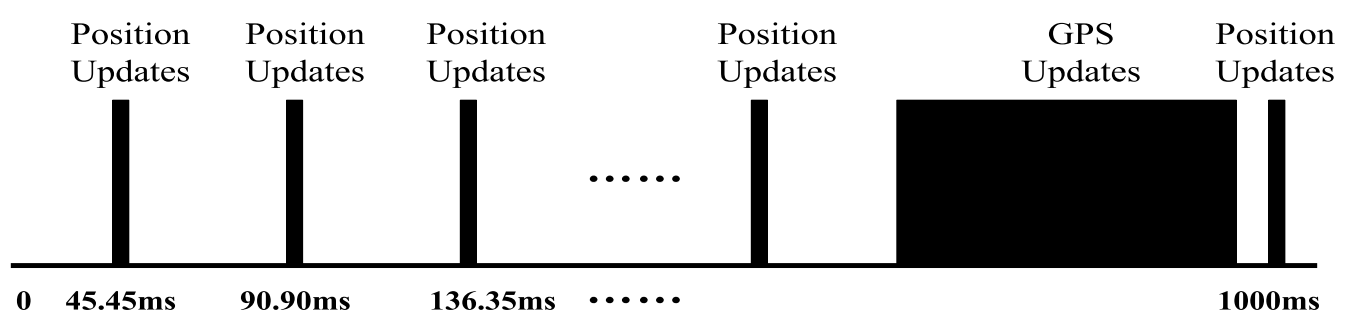

Figure 5.1 A typical time frame within one second of DSP computation.

\subsection{Navigation Board Calibration}

\subsubsection{Test Setup}


The inertial sensors and ADC used in this test were the three-axis accelerometer ADXL335, the single-axis gyroscope LISY300AL and the 14-bit ADC AD7856, which are introduced in Section 5.1. The local gravity used for computing the deterministic error parameters was calculated by the given altitude and latitude provided by the GPS, as follows [47]:

$\mathrm{g}=\mathrm{a} 1 \times\left(1+\mathrm{a} 2 \times \sin ^{2}(\right.$ lat $\left.)\right)+\left(\mathrm{a} 3 \times \sin ^{4}(\right.$ lat $\left.)\right)+\left(\mathrm{a} 4+\mathrm{a} 5 \times \sin ^{2}(\right.$ lat $\left.)\right) \times a l t+\mathrm{a} 6 \times a$ alt $^{2}$

where,

lat and alt denote the latitude in degrees and the altitude in meters respectively,

g denotes the earth's gravity in $\mathrm{m} / \mathrm{s}^{2}$ at the location that is specified by certain latitude and altitude, and

a1, a2, a3, a4, a5 and a6 are normal gravity constants, which is given in Table 5.3.

Table 5.3 Normal gravity constants.

\begin{tabular}{|c|c|c|c|}
\hline Constant & $\mathbf{a 1}$ & $\mathbf{a 2}$ & $\mathbf{a 3}$ \\
\hline Value & 9.7803267714 & 0.0052790414 & 0.0000232718 \\
\hline Constant & $\mathbf{a 4}$ & $\mathbf{a 5}$ & $\mathbf{a 6}$ \\
\hline Value & -0.000003087691089 & 0.000000004397731 & 0.000000000000721 \\
\hline
\end{tabular}

\subsubsection{Multi-Position Static Test Results}

The static test was carried out in the laboratory and the duration of this test at each position was 15 minutes. The average values of these static data from the 3-axis accelerometer were used to calculate 
the deterministic error coefficient matrix $\mathbf{M}$ with the least-square method, according to Equation (3.11). The result is shown below:

$$
M=\left[\begin{array}{cccc}
0.9815 & -0.0008 & -0.0191 & -0.3942 \\
0.0020 & 1.0038 & 0.0043 & 0.4322 \\
0.0330 & 0.0059 & 0.9971 & -0.0122
\end{array}\right]
$$

Based on Equation (5.2), a summary of the deterministic error parameters obtained from the six position static test is shown in Table 5.4.

\subsubsection{Random Error Modeling}

As introduced in Section 3.3, in order to derive the first order GM model parameters, i.e. noise variances and correlation times of MEMS gyroscope and accelerometer, an experimental dataset

Table 5.4 Parameters used in accelerometer's deterministic errors.

\begin{tabular}{|c|c|c||c|c||c|c|}
\hline \multicolumn{1}{|c|}{} & \multicolumn{1}{|c||}{ Acc.X } & \multicolumn{2}{c|}{ Acc.Y } & \multicolumn{2}{c|}{ Acc.Z } \\
\hline \hline Biases (m/s2) & \multicolumn{2}{|c|}{-0.3942} & \multicolumn{2}{c|}{0.4322} & \multicolumn{2}{c|}{-0.0122} \\
\hline Scale Factor Error (\%) & \multicolumn{2}{|c|}{-1.85} & \multicolumn{2}{c|}{0.38} & \multicolumn{2}{c|}{-0.29} \\
\hline $\begin{array}{c}\text { Non-Orthogonalities } \\
\text { (\%) }\end{array}$ & $\mathbf{m . x y}$ & $\mathbf{m . x z}$ & $\mathbf{m . y x}$ & $\mathbf{m . y z}$ & $\mathbf{m . z x}$ & $\mathbf{m . z y}$ \\
\cline { 2 - 7 } & -0.08 & -1.91 & 0.20 & 0.43 & 3.30 & 0.59 \\
\hline
\end{tabular}

was collected from a 2-hour static test to estimate the ACS of inertial sensor errors. By removing the bias offsets from the raw measurements, the ACSs of 2-axis accelerometer and gyroscope can be calculated by Equation (5.3) and they are shown Figure 5.2, 5.5 and 5.7 respectively. From these three plots, the noise variance $\sigma^{2}$ of $1^{\text {st }}$ order GM process can be determined directly at $\mathrm{t}=0$ and correlation time $\tau$ can be obtained when $R(\tau)=\sigma^{2} /$ e. By enlarging Figure 5.2 and 5.5, this procedure is clearly shown in Figure 5.4 and 5.6. As shown in Table 5.5, these parameters were then used to model the stochastic errors of inertial sensors in the field test II. 
Table 5.5 First order GM model parameters used in field test II.

\begin{tabular}{|c|c|c|c|}
\hline & Acc.X & Acc.Y & Gyro.Z \\
\hline Noise Variance $\sigma^{2}$ & 0.002062596 & 0.001597938 & 0.163237989 \\
\hline Correlation Time $\tau(\mathrm{sec})$. & 981.7 & 478 & $<100$ \\
\hline
\end{tabular}

$$
\mathbf{R}_{\mathbf{b b}}(\mathbf{m})=\mathbf{E}[\mathbf{b}(\mathbf{k}) \cdot \mathbf{b}(\mathbf{k}+\mathbf{m})]=\frac{1}{\mathbf{N}-\mathbf{m}} \sum_{\mathbf{k}=\mathbf{1}}^{\mathbf{N}-\mathbf{m}} \mathbf{b}(\mathbf{k}) \cdot \mathbf{b}(\mathbf{k}+\mathbf{m})
$$

where,

$\mathrm{b}(\mathrm{k})$ is a discrete signal with $\mathrm{N}$ sample measurements,

$\mathbf{R}_{\mathrm{bb}}(\mathrm{m})$ is the ACS of the discrete signal $\mathrm{b}(\mathrm{k})$,

$\mathbf{E}[]$ is the mathematical expectation operator, and

$\mathrm{m}$ is the time lag (shift) between samples.

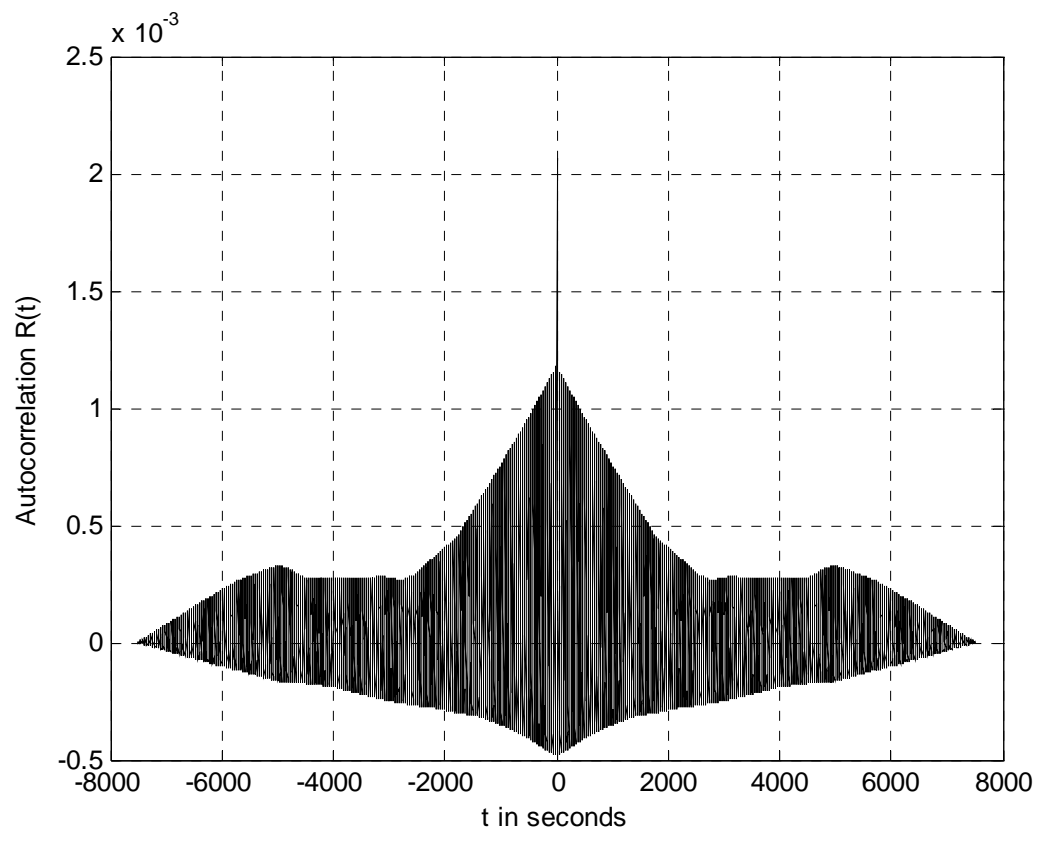

Figure 5.2 The autocorrelation of $\mathrm{x}$-axis accelerometer reading of 2-hour stationary dataset after removing the bias offset. 


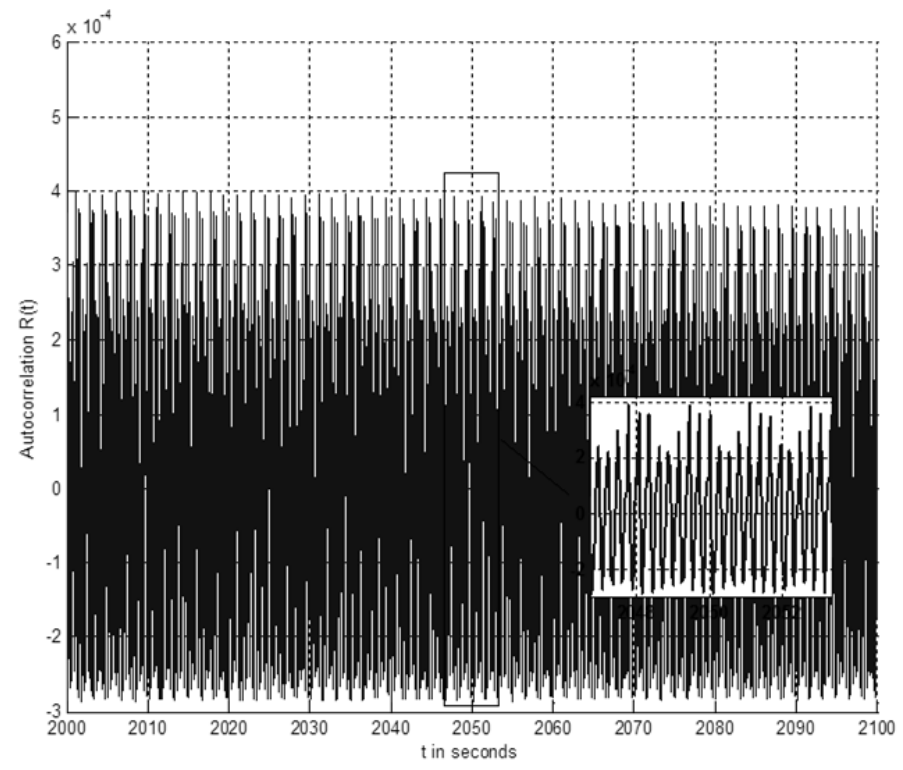

Figure 5.3 Magnified view of Figure 5.2.

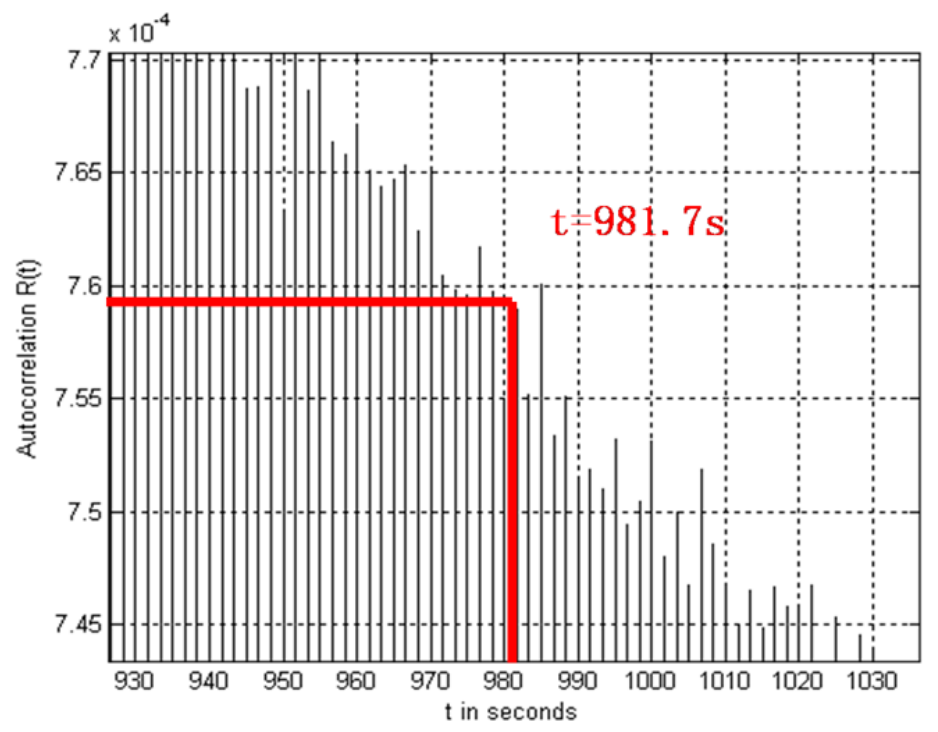

Figure 5.4 Correlation time $\boldsymbol{\tau}$ of $\mathrm{X}$-axis accelerometer

$$
\text { at }(\tau)=\frac{\sigma^{2}}{\mathrm{e}}=\mathbf{7 . 5 9} \times 10^{-4} \text {. }
$$




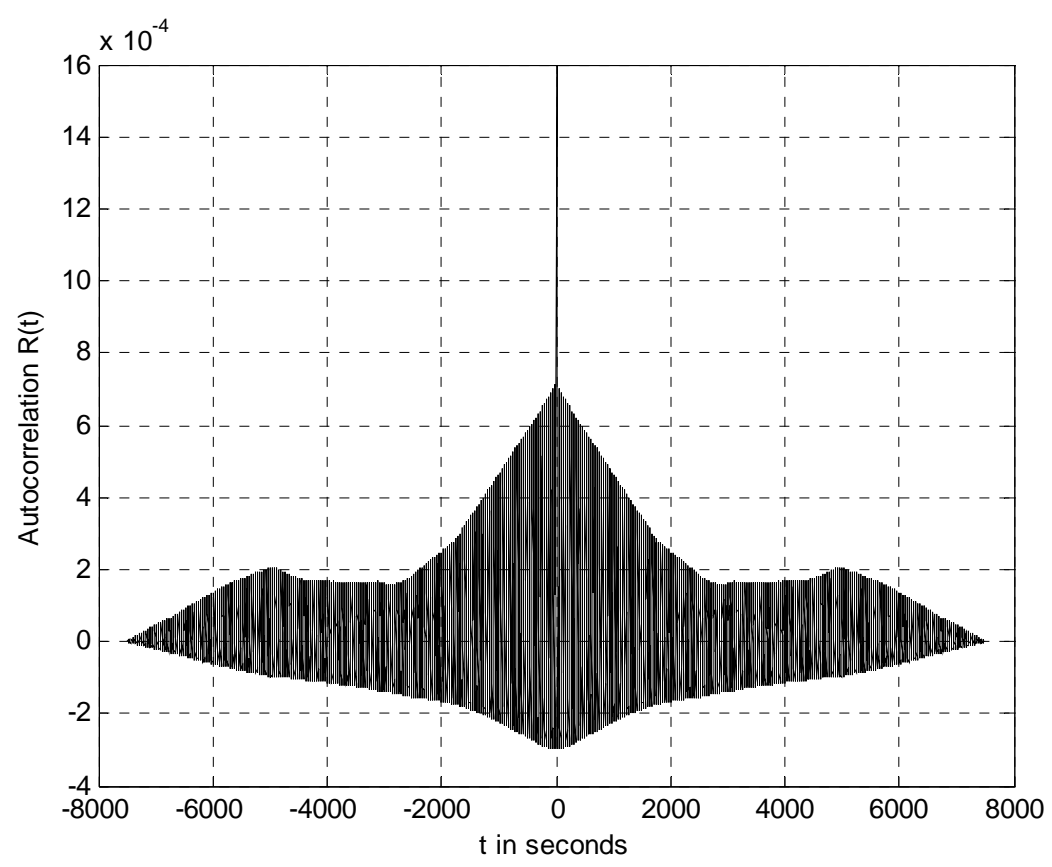

Figure 5.5 The autocorrelation of y-axis accelerometer reading of 2-hour stationary dataset after removing the bias offset.

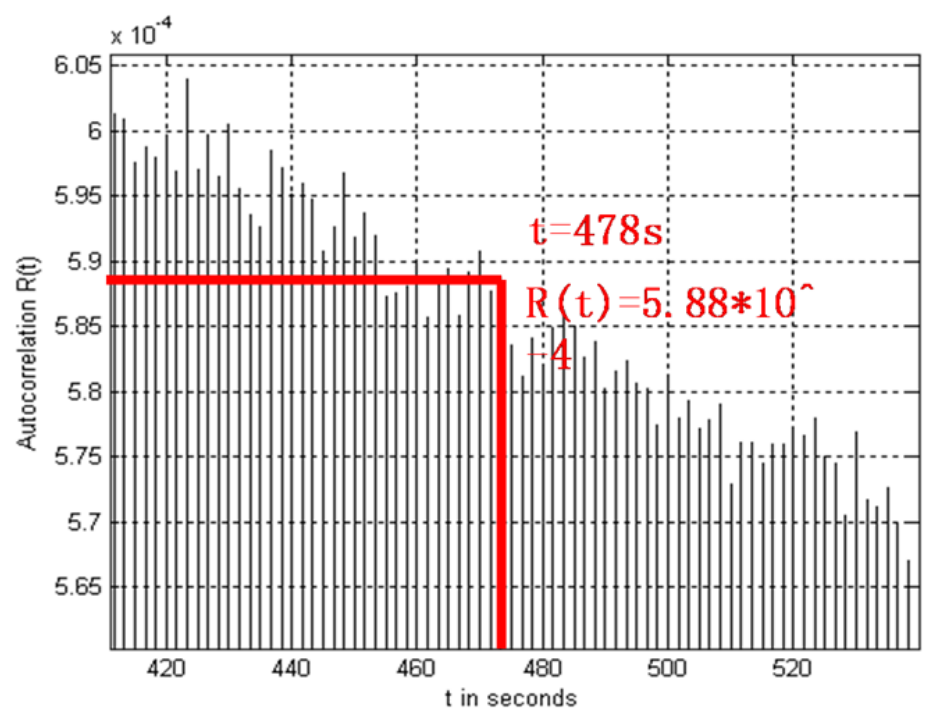

Figure 5.6 Correlation time $\boldsymbol{\tau}$ of $\mathrm{Y}$-axis accelerometer

$$
\text { at } \mathbf{R}(\tau)=\frac{\sigma^{2}}{\mathrm{e}}=\mathbf{5 . 8 8} \times 10^{-4}
$$




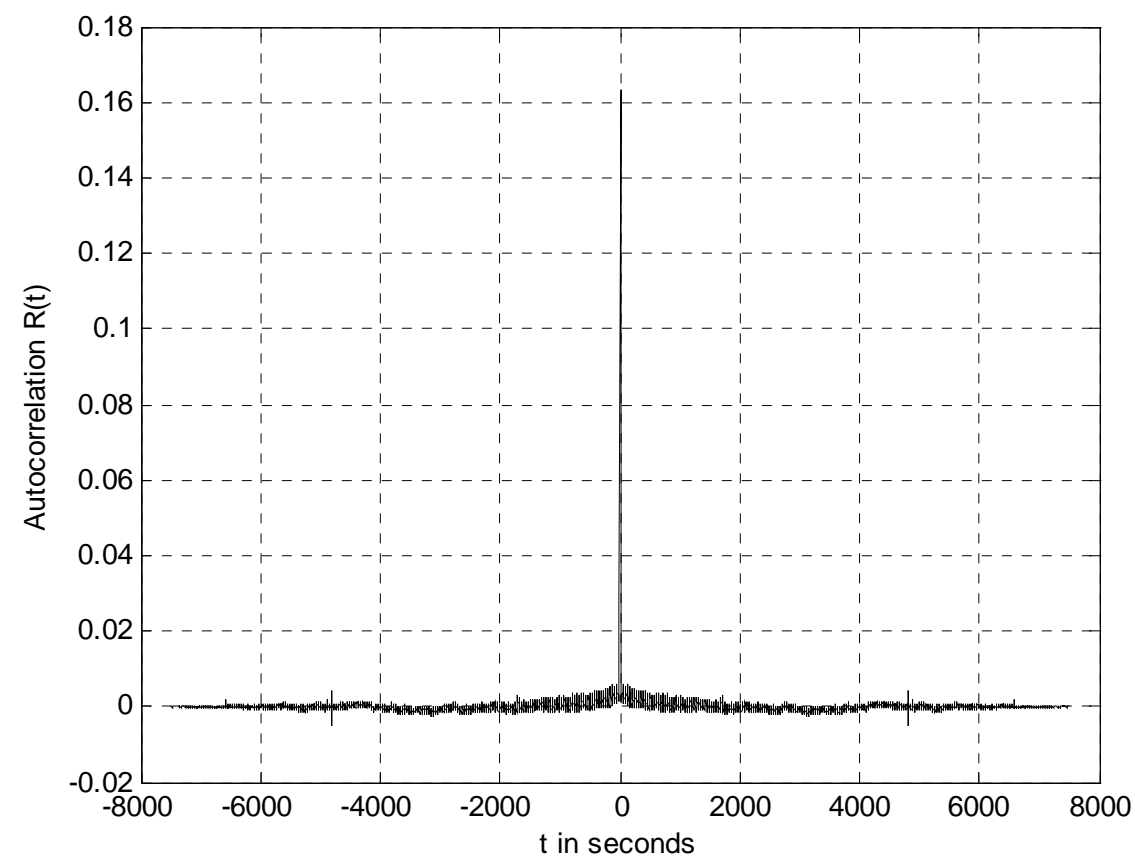

Figure 5.7 The autocorrelation of z-axis gyroscope reading of 2-hour stationary dataset after removing the bias offset.

\subsection{Open Field Tests}

Data from open area tests are being studied and analyzed. Besides, trajectories generated by the navigation board in the real-time scenario are also depicted in this section. Dataset I was provided by Walid Farid Abd El Fatah from Dr. Aboelmagd Noureldin's laboratory at Royal Military College (RMC), Kingston, Canada. This test is to assess the positioning accuracy of the 2D mechanization and the Kalman filter algorithm with accurate acceleration and angular rate inputs. Moreover, field tests using the prototype system, which is introduced in Section 5.1, were conducted and repeated for many times. Dataset II is a good example of those test results. The third test is to evaluate the performance of the proposed navigation board that is introduced in Chapter 4. The navigation performance to bridge GPS outage has been verified by all those experiments. 


\subsubsection{Field Test I}

\subsubsection{Test Setup and Description}

To examine the effectiveness of the navigation frame INS mechanization and the aforementioned Kalman filter for 2D INS/GPS integration solution, field test I was carried out with MEMS-grade IMU and wheel encoders to provide the angular rate and acceleration of the vehicle. The vehicle used in this test is a three-wheel robot and its movement is controlled by a joystick and the motors are powered by four rechargeable storage batteries. This vehicle was equipped with the Xbow IMU300CC-100 and wheel encoders to provide the INS raw measurements. The Xbow IMU was used to measure the heading angle and the forward acceleration was calculated with respect to the velocity measurements from the wheel encoders. The specification for IMU300CC-100 is given in Table 5.6.

Table 5.6 Specifications for XBow IMU300CC-100.

\begin{tabular}{|l|c|}
\hline \multicolumn{2}{|c|}{ Xbow IMU300CC-100 } \\
\hline \hline Specification & Value \\
\hline \hline Update Rate (Hz) & $>100$ \\
\hline Range ( $/$ sec) & \pm 100 \\
\hline Bias ( $/$ sec) & $< \pm 2.0$ \\
\hline Scale Factor Accuracy (\%) & $<1$ \\
\hline Non-Linearity (\% FS) & $<0.3$ \\
\hline Resolution ( $/$ sec) & $<0.025$ \\
\hline Bandwidth (Hz) & $>25$ \\
\hline Random Walk ( $/ \mathbf{h r} \mathbf{1} / \mathbf{2})$ & $<2.25$ Typical \\
\hline
\end{tabular}


Table 5.7 GPS/IMU specifications of the reference system.

\begin{tabular}{|l|c|c|}
\hline \multicolumn{3}{|c|}{ IMU HG1700 } \\
\hline \hline Specifications & Gyroscope & Accelerometer \\
\hline \hline Range & $\pm 1,000 \mathrm{deg} / \mathrm{sec}$ & $\pm 50 \mathrm{~g}$ \\
\hline Bias & $1.0 \mathrm{deg} / \mathrm{hr}$ & $1.0 \mathrm{mg}$ \\
\hline Scale Factor & $150 \mathrm{ppm}$ & $300 \mathrm{ppm}$ \\
\hline $\begin{array}{l}\text { Angle/Velocity Random } \\
\text { Walk }\end{array}$ & $0.125 \mathrm{deg} / \sqrt{\mathrm{hr}}$ & $500 \mathrm{ppm}$ \\
\hline Linearity & No spec \\
\hline \multicolumn{2}{|c||}{ GPS NovAtel OEM4 } \\
\hline \hline Technical Specifications \\
\hline \hline 24-Channel L1/L2 C/A Code with full Carrier Tracking \\
\hline Pulse Aperture Correlator ${ }^{\mathrm{TM}}$ \\
\hline Data Rate 20 Hz \\
\hline Position Accuracy DGPS (L1, C/A) 0.45 m CEP \\
\hline Velocity Accuracy 0.03 m/s RMS \\
\hline
\end{tabular}

The duration of this test is 9 minutes and 41 seconds and the overall distance was approximately 400 meters. The reference system used in this test to access the navigation solution is composed of the Novatel OEM4 GPS receiver and the Honeywell HG1700 tactical grade IMU. The specification of the reference system is given by Table 5.7 .

\subsubsection{Results and Analysis}

By comparing the measurements and outputs from the reference system introduced in the previous section with the outputs provided by the integrated filter, this section will discuss the performance of the navigation algorithm in terms of longitude, latitude, velocities and the heading angle. The emphasis of this section is placed on the estimation accuracies of the navigation system during the absence of GPS updates.

As mentioned in Chapter 1, to maintain reliable performance of the KF prediction, the GPS aiding information should be available at all times. However, the sky is often masked by various 
obstructions such as trees and buildings. This will result in the partial or complete loss of satellite visibility. In these cases, it is important to know the impact on the navigation results when a GPS update is absent and the filter works in a full prediction mode. Therefore, several simulated GPS outages were artificially conducted into the trajectory to assess the prediction accuracy of the navigation system.

Figure 5.8 demonstrates and compares the trajectories provided by the integrated filter and the reference system. As shown in this figure, the movement of the vehicle was simple: it only moved along straight lines and turned with 90-degree angles in the test. There are four GPS outages with 60second durations in the trajectory. Two of them happened during the straight-line motion and the other two outages covered the first and the third cornering motions.

Figure 5.9 and 5.12 give a further representation of the position errors and velocity errors respectively, based on the reference results. Figure 5.9 shows that when the GPS signals were not available, the position errors accumulated with the course of time until the system regained the GPS updates. The only exception is the second GPS signal gap. The errors reached the maximum value when the vehicle was turning the corner. The errors started to reduce when the vehicle ran back into a straight line.

In addition, the horizontal velocities and the yaw angles from the EKF results and the reference outputs are depicted in Figure 5.10, 5.11 and 5.13. It may be noticed that when GPS updates are available, both the north velocity and the east velocity obtained from the EKF and the reference system closely overlap, which results in very few position errors, as shown in Figure 5.8 and 5.9. 
Figure 5.13 clearly shows that the yaw angle calculated by the integrated filter was almost the same as the reference outputs, even during the GPS outages.

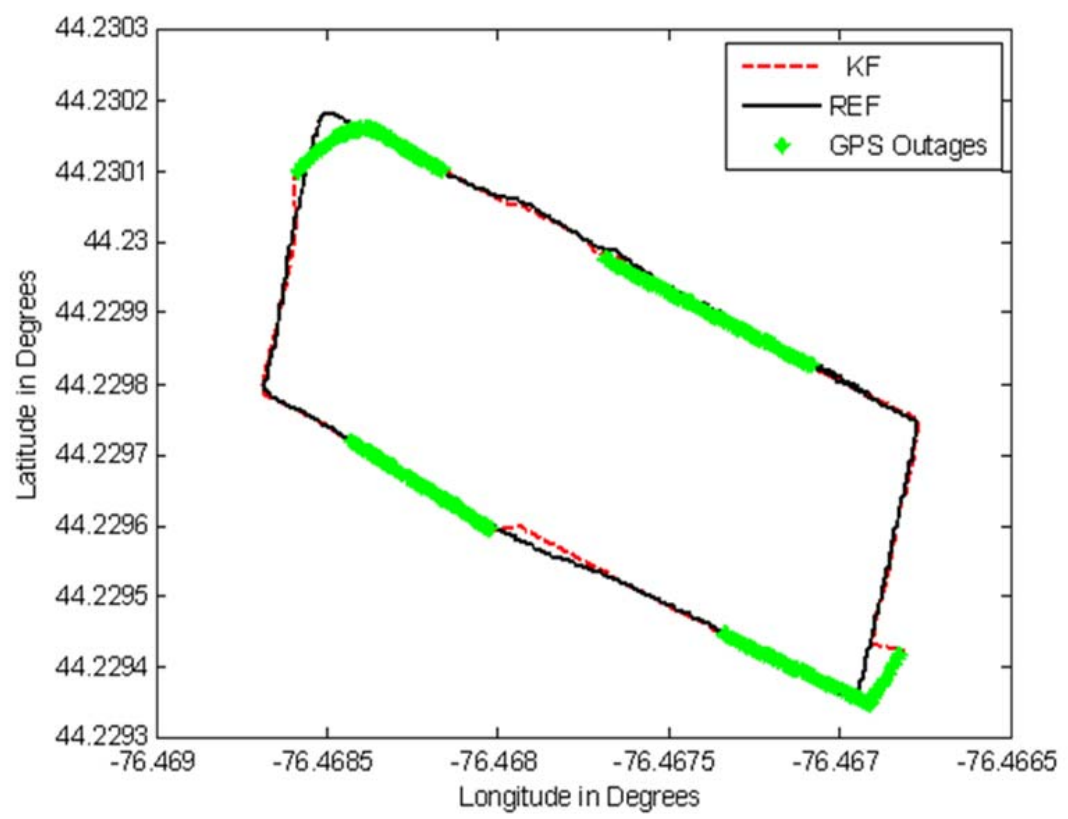

Figure 5.8 Field test I: Reference trajectory and KF trajectory with 4 simulated GPS outages.

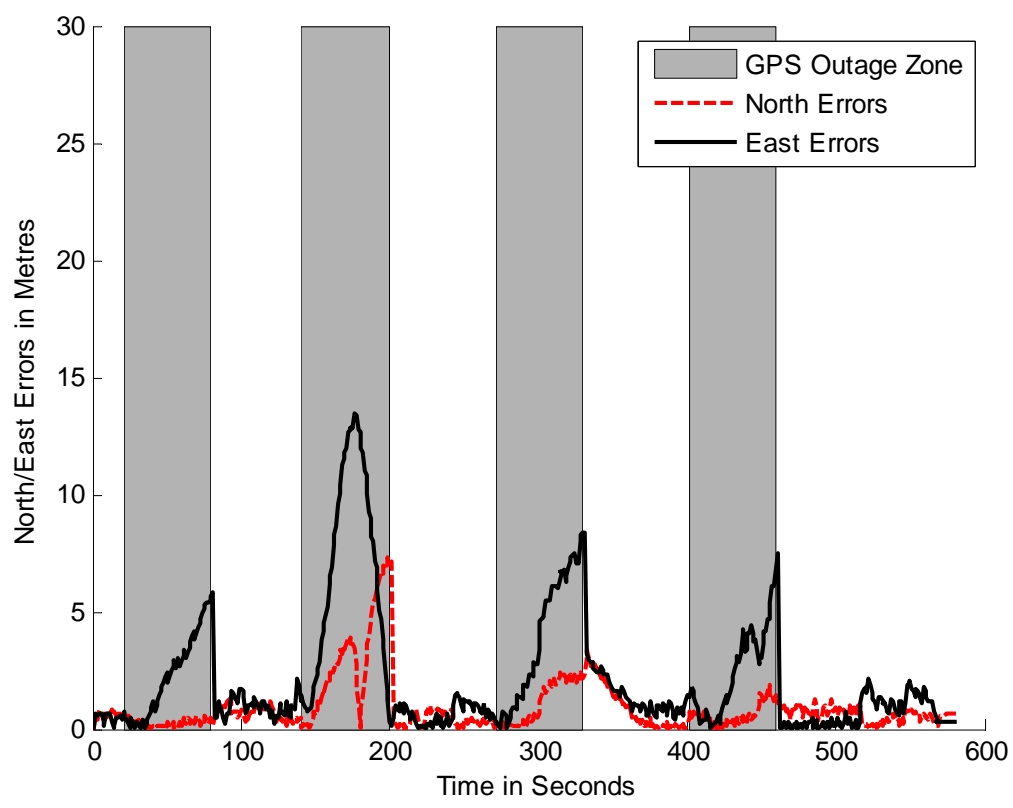

Figure 5.9 Field test I: North/East position errors. 


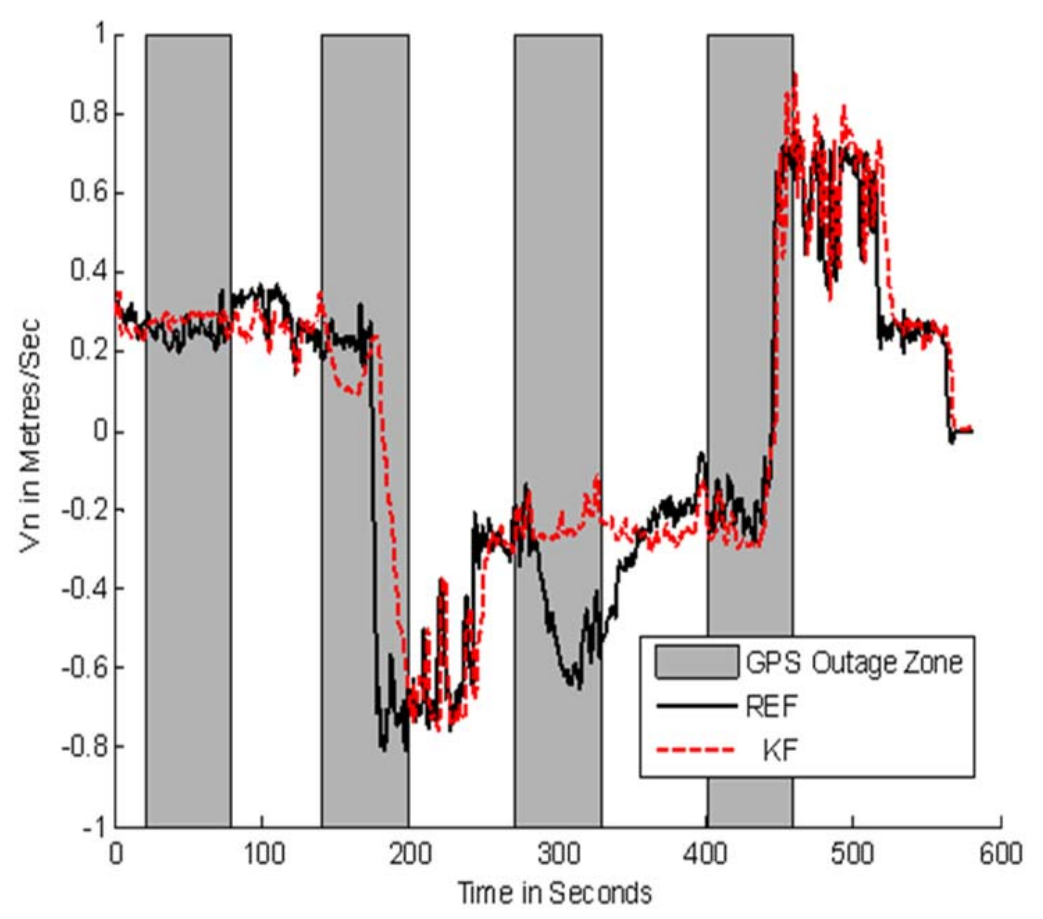

Figure 5.10 Field test I: Reference north velocity and KF north velocity.

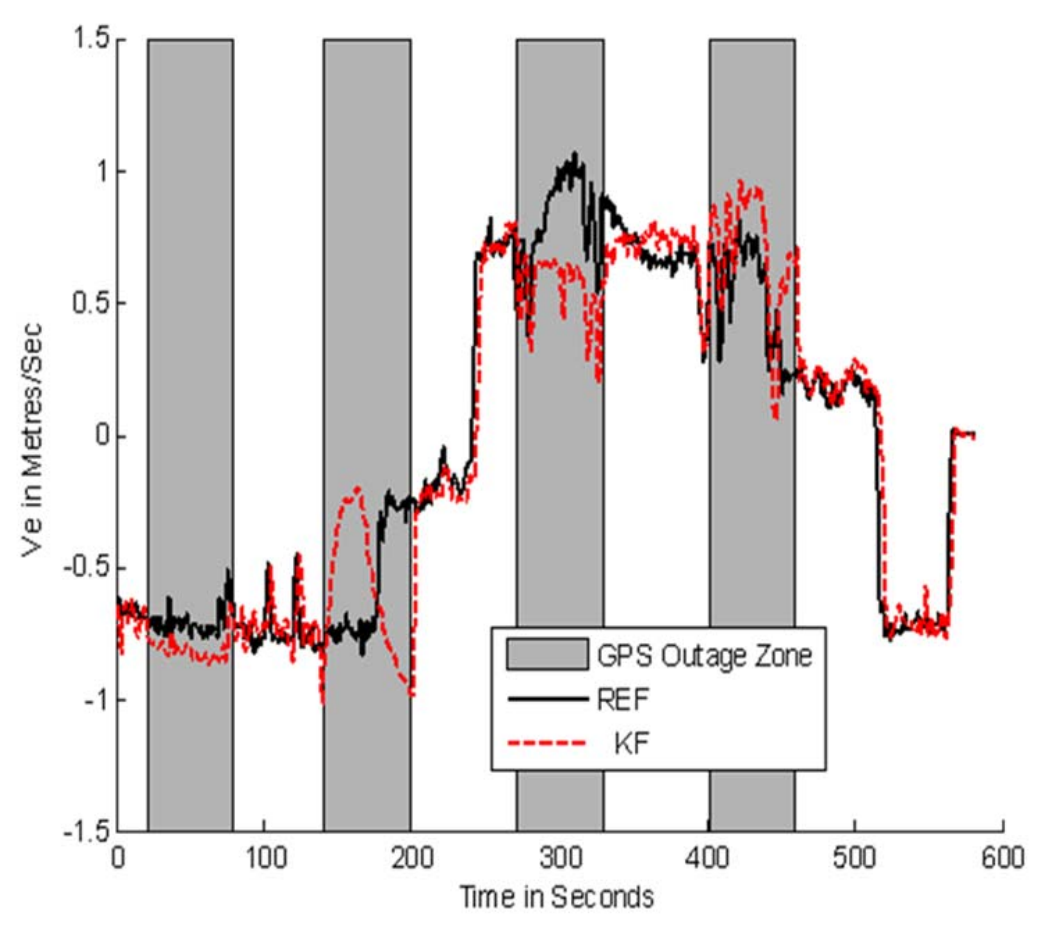

Figure 5.11 Field test I: Reference east velocity and KF east velocity. 


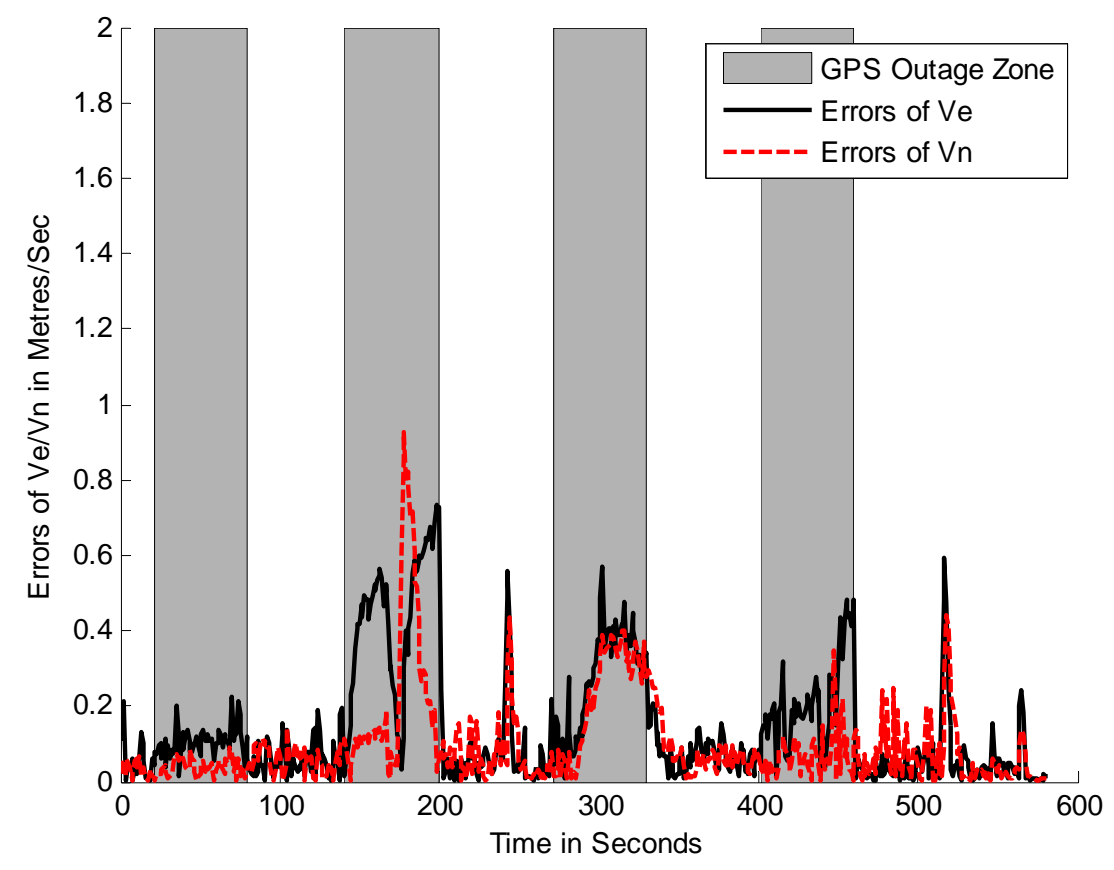

Figure 5.12 Field test I: East/North velocity errors.

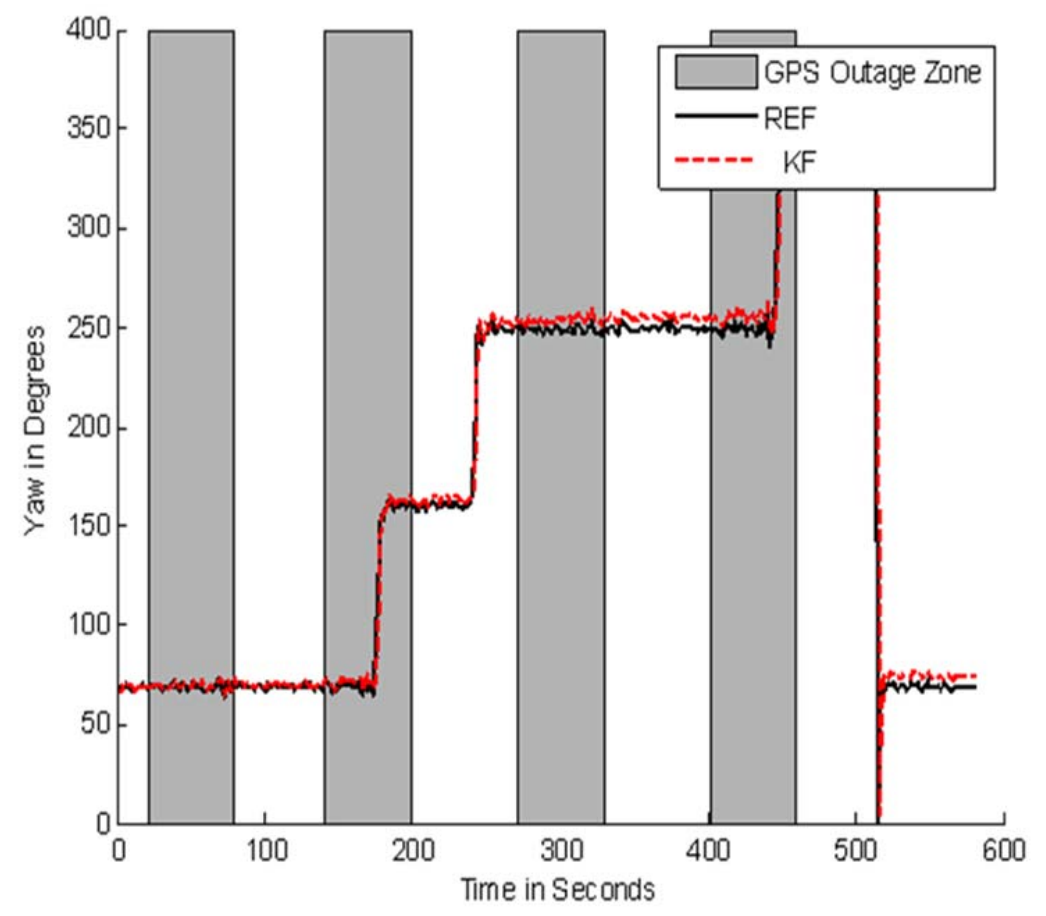

Figure 5.13 Field test I: Reference yaw and KF yaw. 
Table 5.8 shows the mean, RMS and maximum values of the position and velocity errors provided by EKF during four GPS outages. Generally, it can be determined from this table that the positioning results during 60-second GPS outage periods are accurate. The RMS values are less than 9 meters and the maximum errors are less than 14 meters.

Table 5.8 Field test I: Position/Velocity errors during GPS outages.

\begin{tabular}{|l|c|c|c|c|}
\hline & Outage 1 & Outage 2 & Outage 3 & Outage 4 \\
\hline North Position & Mean 0.270 & Mean 2.969 & Mean 1.131 & Mean 0.622 \\
Errors & RMS 0.320 & RMS 3.675 & RMS 1.461 & RMS 0.776 \\
\hline East Position & Max. 0.709 & Max. 7.282 & Max. 2.430 & Max. 1.862 \\
Errors & RMS 2.868 & RMS 8.133 & RMS 4.751 & RMS 3.047 \\
in Meters & Max. 5.638 & Max. 13.450 & Max. 8.362 & Max. 6.801 \\
\hline Position Errors & Mean 2.329 & Mean 7.918 & Mean 4.034 & Mean 2.545 \\
in Meters & RMS 2.886 & RMS 8.924 & RMS 4.971 & RMS 3.144 \\
& Max. 5.646 & Max. 13.881 & Max. 8.637 & Max. 6.959 \\
\hline North Velocity & Mean 0.038 & Mean 0.238 & Mean 0.222 & Mean 0.069 \\
Errors in M/Sec & RMS 0.044 & RMS 0.342 & RMS 0.262 & RMS 0.092 \\
& Max. 0.093 & Max. 0.927 & Max. 0.402 & Max. 0.350 \\
\hline East Velocity & Mean 0.104 & Mean 0.436 & Mean 0.275 & Mean 0.212 \\
Errors in M/Sec & RMS 0.110 & RMS 0.476 & RMS 0.306 & RMS 0.242 \\
& Max. 0.225 & Max. 0.733 & Max. 0.568 & Max. 0.483 \\
\hline
\end{tabular}

\subsubsection{Field Test II}

\subsubsection{Test Description}

The navigation system used in this test is composed of ADXL335, LISY300AL, AD7856 and GPS

LEA-5H. Details are mentioned previously in Section 5.1. The DSP used in this test is 
TMS320VC5505EVM, which is the evaluation module of C5505 from TI. Inertial sensors were mounted on a 4-wheel cart. The GPS antenna was mounted on the top layer of this cart. Data acquisition was carried out using DSP to read both INS and GPS data. A laptop provided the power for the whole prototype board and recording the data sent from DSP C5505.

This field test was carried out along the racetrack around the soccer field, on the main campus of Queen's University in Kingston, ON, which is an open area with a sufficient number of satellites available throughout the entire time period of the test. The average speed for this test was 1.45 meters per second. Vibration of the inertial sensors was minimal due to the flatness of the ground. The test duration was 4 minutes and 41 seconds and the total distance travelled was approximately 400 meters.

\subsubsection{Results and Analysis}

Both trajectories obtained from the KF with and without the use of NHC are depicted in Figure 5.14. Similarly to the first test, four 20 -second GPS outage scenarios were simulated at different locations along the trajectory to evaluate the performance of the navigation solution when GPS signals are absent. In addition, the position/velocity errors of the test results with and without the use of NHC are given and compared in Figure 5.15, 5.16, 5.19 and 5.20. It is clearly shown that the errors increased rapidly and with high amplitude during GPS outages.

Figure 5.17, 5.18, 5.21 compare north velocity, east velocity and the yaw angle obtained from the three scenarios (GPS, KF, KF with the use of NHC) respectively. In Figure 5.17 and 5.18, it is clearly shown that the velocities provided by the integration filter deviate from the GPS outputs during the signal outages. 


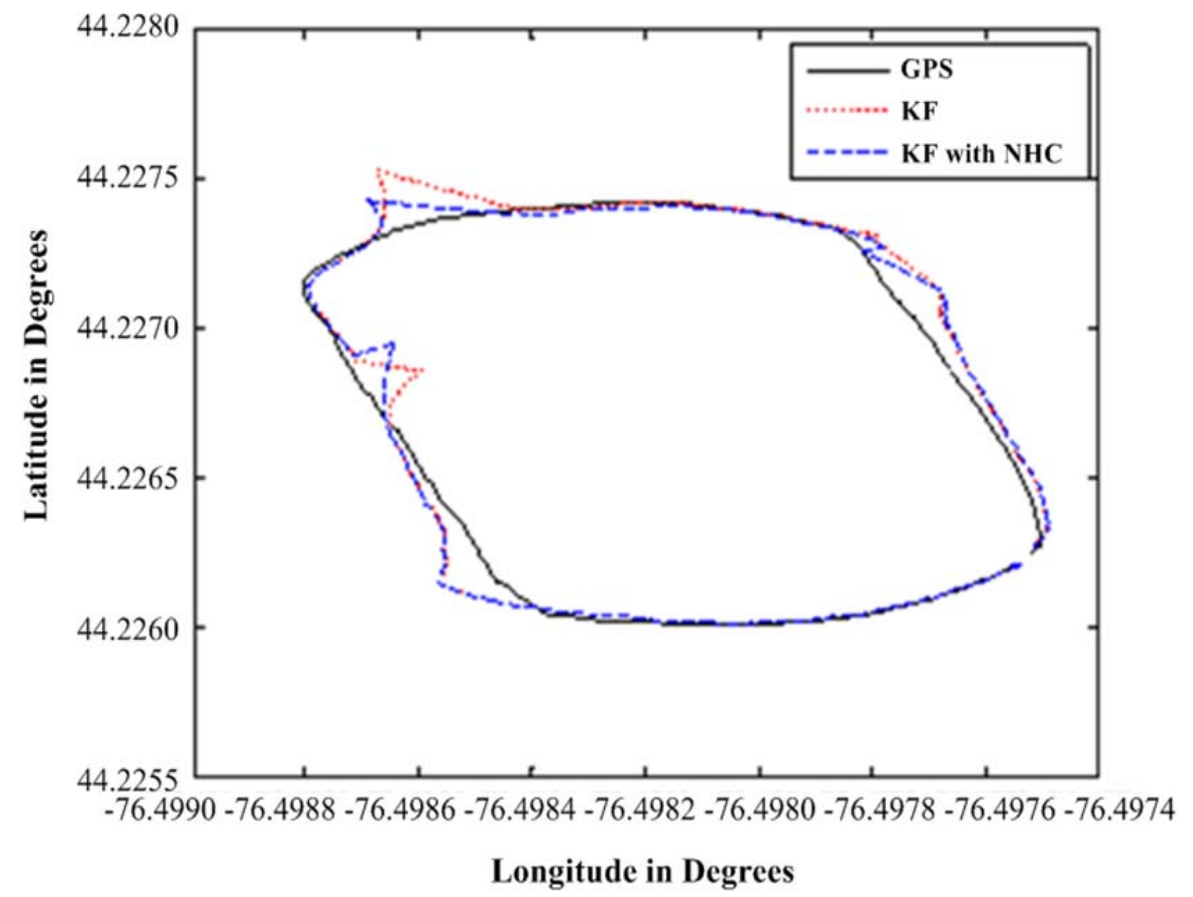

Figure 5.14 Field test II: GPS trajectory and KF trajectories with 4 simulated GPS outages.

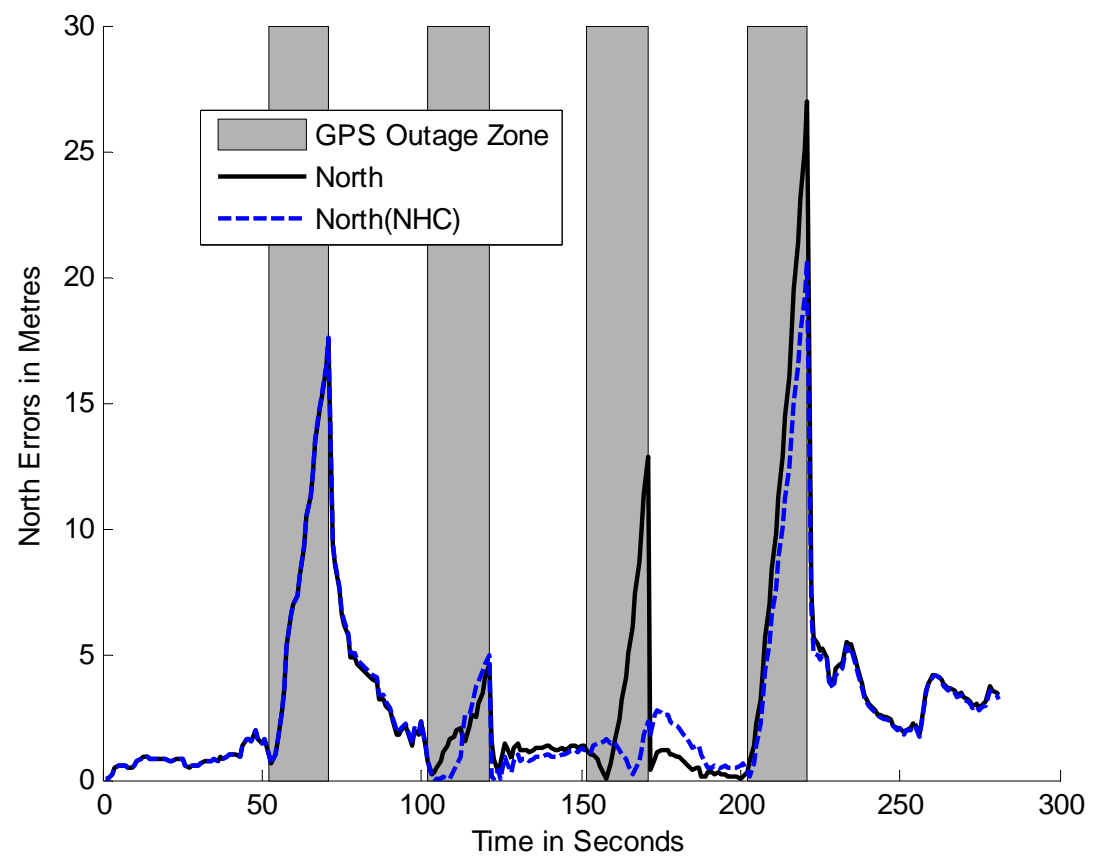

Figure 5.15 Field test II: North position errors. 


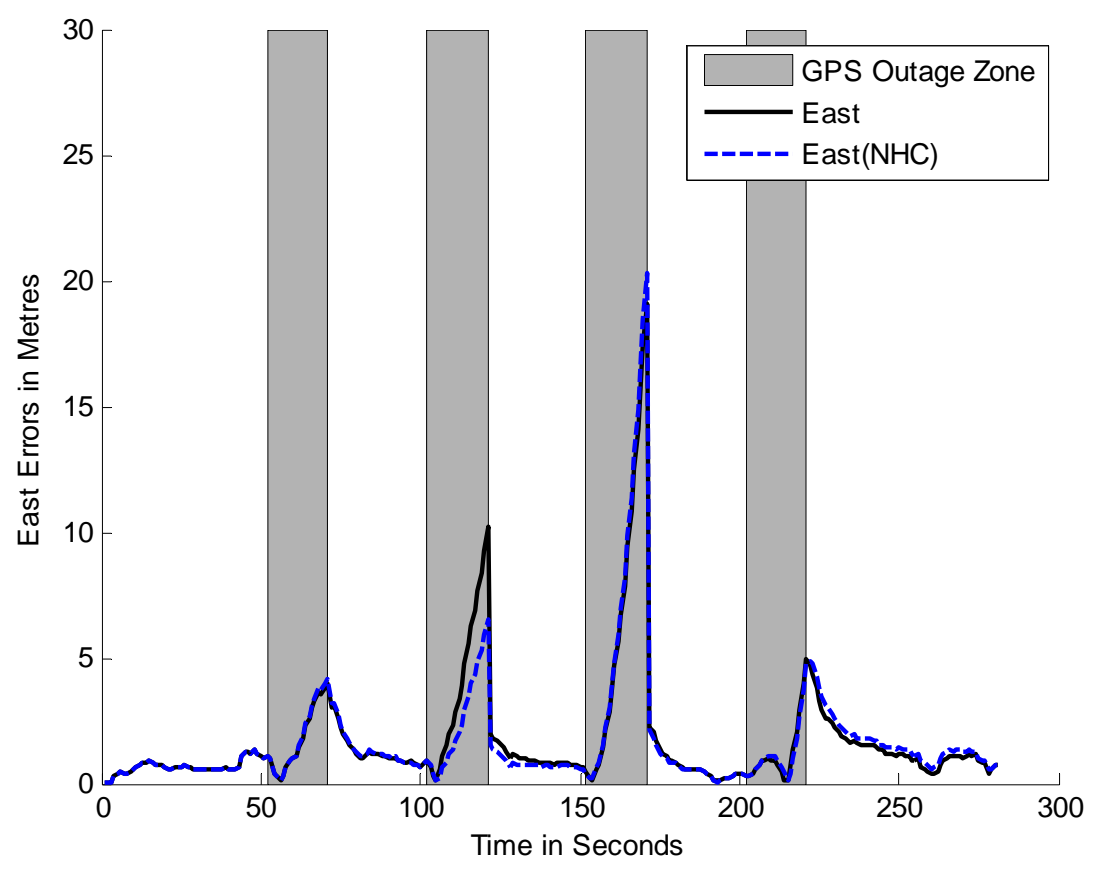

Figure 5.16 Field test II: East position errors.

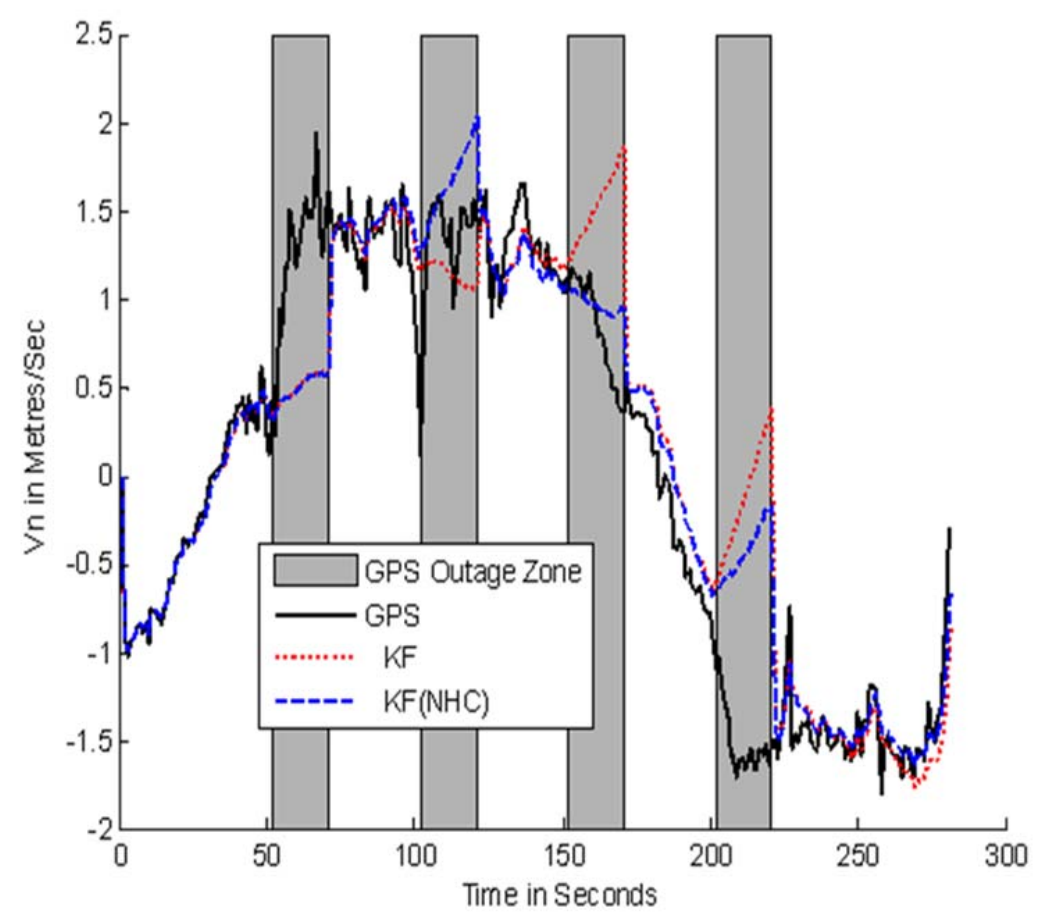

Figure 5.17 Field test II: GPS north velocity and KF north velocity. 


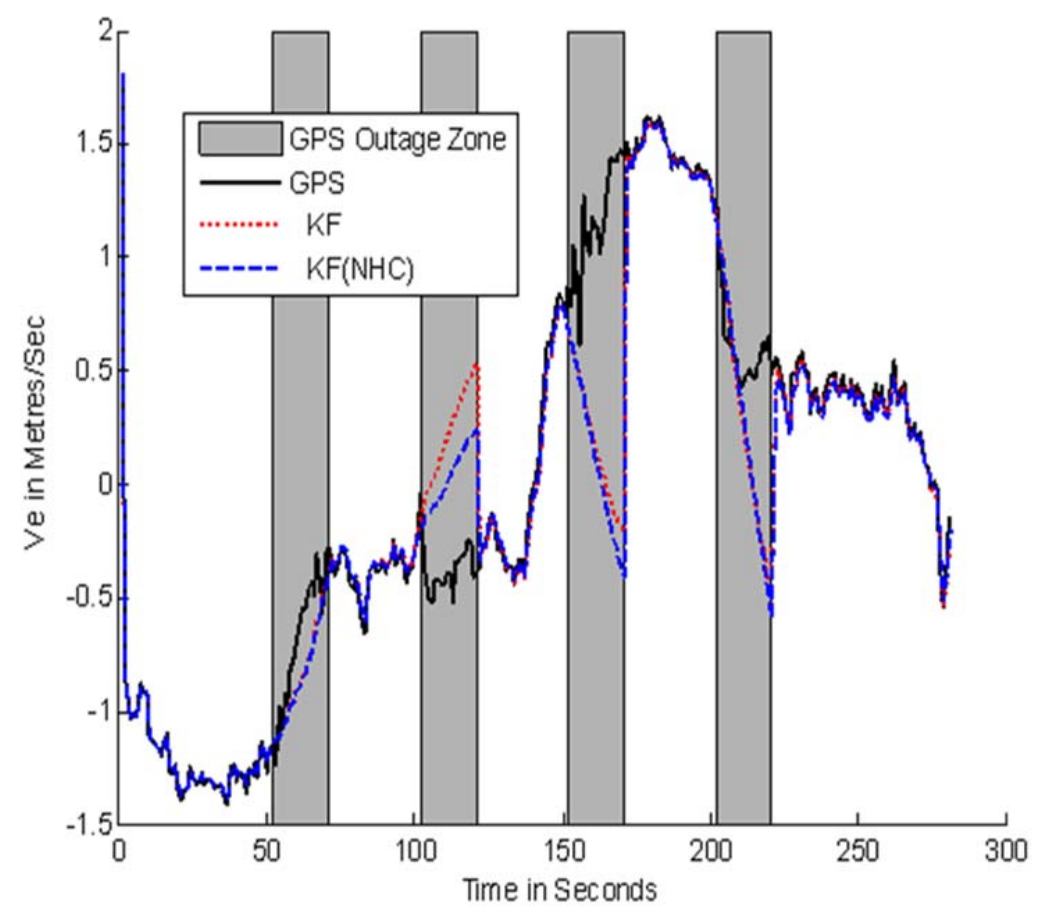

Figure 5.18 Field test II: GPS east velocity and KF east velocity.

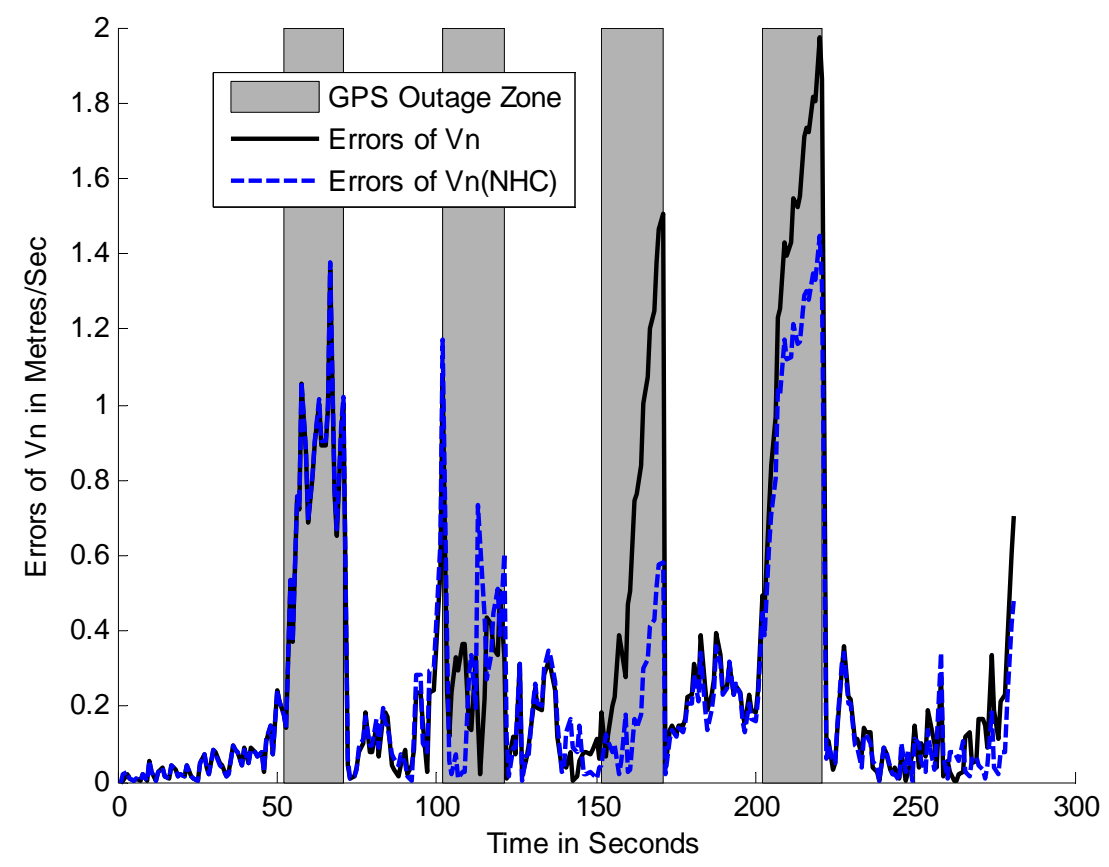

Figure 5.19 Field test II: north velocity errors. 


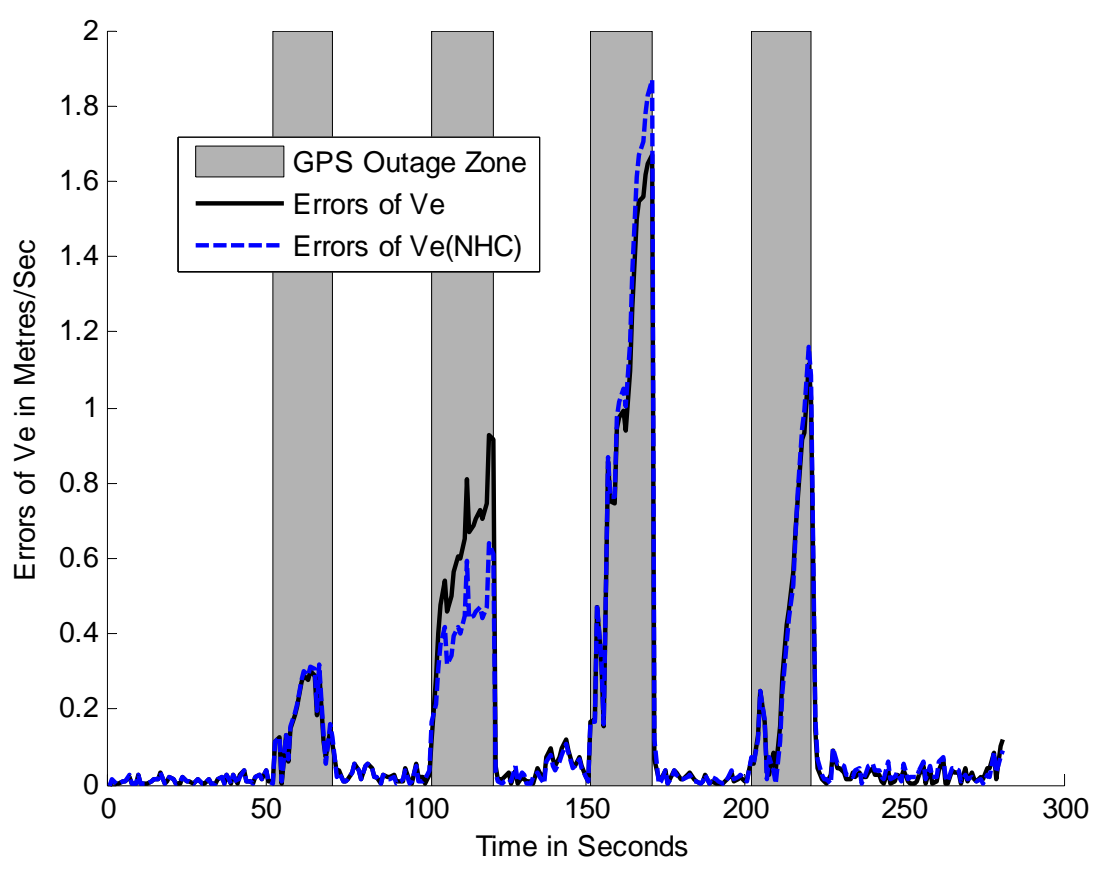

Figure 5.20 Field test II: east velocity errors.

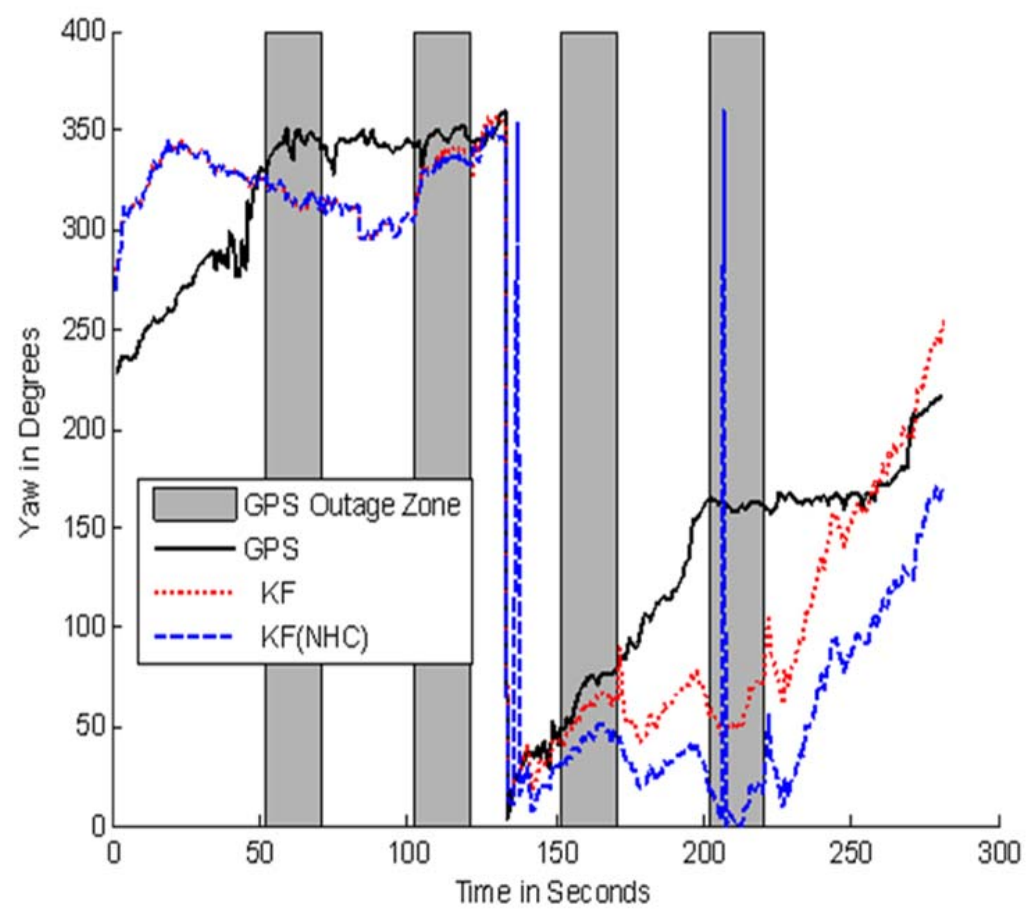

Figure 5.21 Field test II: GPS yaw and KF yaw. 
The statistics of the position and velocity differences between integrated results and GPS outputs during four simulated GPS signal gaps are summarized in Table 5.9. The RMS values of the position errors are less than 14.5 meters while the maximum value of the position errors is 27.42 meters. The RMS values of the velocity errors of these four GPS outages are not greater than 1.44 meters/second. As discussed in previous chapters, the accuracy of the Kalman filter is limited by the noise level of the MEMS sensor input. Therefore, the position/velocity errors grew rapidly during GPS outages. As mentioned in Section 3.4, non-holonomic constraints can be used to improve the accuracy of the loosely coupled GPS/INS integration system. Table 5.10 compiles the corresponding differences

Table 5.9 Field test II: Position/Velocity errors during GPS outages.

\begin{tabular}{|l|c|c|c|c|}
\hline & Outage 1 & Outage 2 & Outage 3 & Outage 4 \\
\hline Northing Position & Mean 8.25 & Mean 1.89 & Mean 3.96 & Mean 11.56 \\
Errors & RMS 9.83 & RMS 2.23 & RMS 5.58 & RMS 14.28 \\
in Meters & Max. 17.44 & Max. 4.61 & Max. 12.81 & Max. 26.97 \\
\hline Easting Position & Mean1.81 & Mean 3.92 & Mean 6.82 & Mean 1.23 \\
Errors & RMS 2.23 & RMS 5.03 & RMS 9.12 & RMS 1.76 \\
in Meters & Max. 3.98 & Max. 10.21 & Max. 19.04 & Max.4.94 \\
\hline Position Errors & Mean 8.49 & Mean 4.40 & Mean 7.98 & Mean 11.65 \\
in Meters & RMS 10.08 & RMS 5.50 & RMS 10.69 & RMS 14.39 \\
& Max. 17.89 & Max. 11.21 & Max. 22.95 & Max. 27.42 \\
\hline Northing Velocity & Mean 0.78 & Mean 0.32 & Mean 0.70 & Mean 1.37 \\
Errors in M/Sec & RMS 0.83 & RMS 0.38 & RMS 0.85 & RMS 1.44 \\
& Max. 1.36 & Max.1.08 & Max. 1.51 & Max.1.97 \\
\hline Easting Velocity & Mean 0.17 & Mean 0.61 & Mean 0.97 & Mean 0.41 \\
Errors in M/Sec & RMS 0.19 & RMS 0.64 & RMS 1.09 & RMS 0.55 \\
& Max. 0.30 & Max. 0.93 & Max. 1.67 & Max. 1.11 \\
\hline
\end{tabular}


Table 5.10 The accuracy improvement with NHC.

\begin{tabular}{|l|c|c|c|c|}
\hline & Outage 1 & Outage 2 & Outage 3 & Outage 4 \\
\hline Latitude Error & Mean 0.05311 & Mean -0.15141 & Mean -2.73988 & Mean -2.71550 \\
in Meters & RMS 0.07152 & RMS 0.25123 & RMS -4.27284 & RMS -3.29470 \\
& Max. 0.16383 & Max. 0.37841 & Max. -10.46811 & Max. -6.41486 \\
\hline \multirow{2}{*}{ Longitude Error } & Mean 0.06345 & Mean -1.43684 & Mean 0.35443 & Mean -0.02630 \\
& RMS 0.08462 & RMS -1.84654 & RMS 0.51679 & RMS -0.08213 \\
& Max. 0.18479 & Max. -3.63789 & Max. 1.29497 & Max. -0.16886 \\
\hline Position Error & Mean 0.06614 & Mean -1.31906 & Mean -0.44483 & Mean -2.68400 \\
& RMS 0.08871 & RMS -1.46655 & RMS -0.96712 & RMS -3.27541 \\
\hline North Velocity & Max. 0.20141 & Max. -2.95220 & Max. -2.47791 & Max. -6.31721 \\
Error in M/Sec & RMS 0.00960 & RMS 0.06688 & RMS -0.55773 & RMS -0.33708 \\
& Max. 0.01426 & Max. 0.09853 & Max. -0.92419 & Max. -0.52490 \\
\hline East Velocity & Mean 0.00820 & Mean -0.18625 & Mean 0.06667 & Mean -0.00213 \\
Error in M/Sec & RMS 0.00923 & RMS -0.20072 & RMS 0.08750 & RMS 0.01225 \\
& Max. 0.01536 & Max. -0.28659 & Max. 0.20073 & Max. 0.04880 \\
\hline
\end{tabular}

between the position/velocity error statistics provided by the Kalman filter with and without the use of NHC to assess the accuracy improvement. Based on the data set shown in this table, we can see that both velocity and position estimations of the KF with NHC give better results in comparison with the KF algorithm that does not use NHC, although in some cases they provide a comparable level of accuracy. It can be concluded that non-holonomic constraints guarantee an improved performance during short term GPS outages.

\subsubsection{Field Test III}

\subsubsection{Test Description}


Several field tests were carried out to evaluate the performance of the navigation board which is introduced in Chapter 4. The first 3 tests shown in this section were conducted by a person on foot, at the same place as field test II (the racetrack around the soccer field, on the main campus of Queen's University). The other 3 road trajectory tests were carried out in a land-vehicle, near the main campus of Queen's University, Kingston.

In these tests, GPS LEA-5H was integrated with the navigation board and inertial sensors ADIS16003, ADIS16060 and HMC6352 were used to provide the acceleration, the angular rate, and the heading angle respectively. Another GPS $\mu$-blox EVK-5H was used as a reference to compare the positioning performance with the navigation board. A laptop provided the power for the GPS EVK5H and CC2430EM-SmartRF04EB which is used to receive the position data sent from the navigation board. The equipment setup is shown in Figure 5.22.

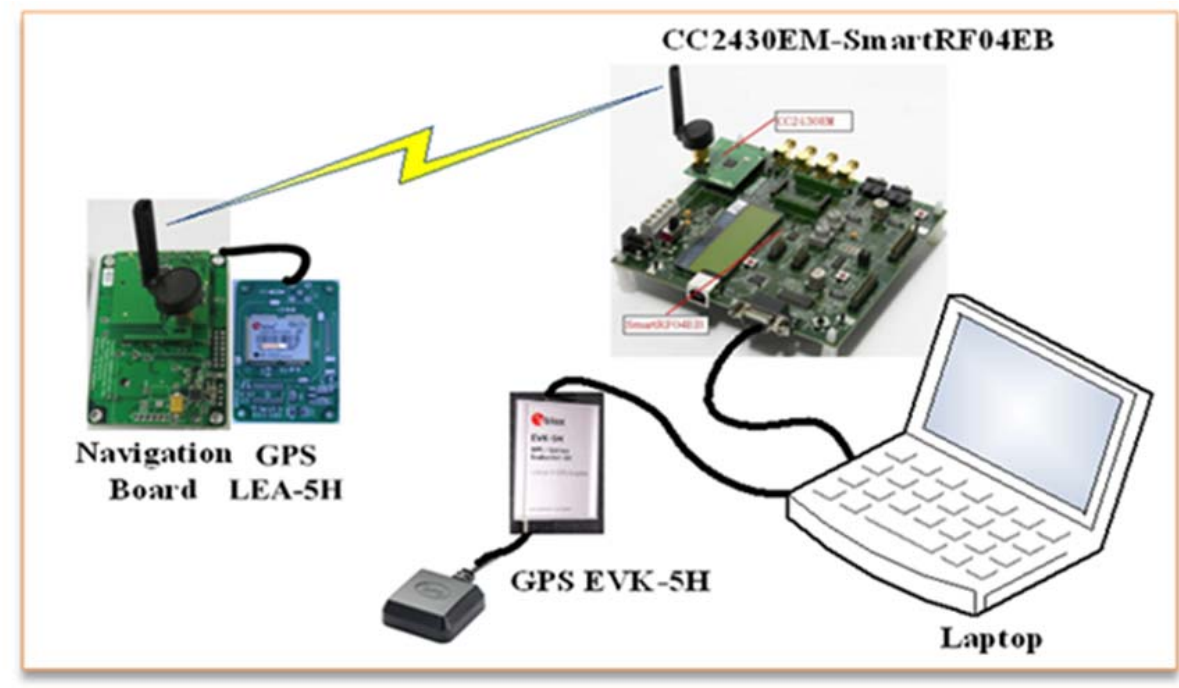

Figure 5.22 Equipment setup for the field test.

\subsubsection{Test Results}


Three datasets of those trajectories obtained from the GPS EVK-5H and the navigation board with the on-foot scenario are depicted in Figure 5.23, Figure 5.24 and Figure 5.25. Several GPS outages took place at different locations along the trajectory and the average outage duration was around 30 seconds. Figure 5.26, Figure 5.27 and Figure 5.28 show the trajectories for the in-vehicle scenario. Table 5.12 and Table 5.13 summarize the position differences between the navigation board outputs and GPS reference outputs during all the GPS outages. The average position errors of the outages in each trajectory are also included. Although the position errors exceed 20 meters during 30-second GPS outages due to the use of the low-cost MEMS-based inertial sensors, the results still illustrate the reliability of this proposed hardware system in providing accurate navigation solution.

Table 5.11 Specifications for ADIS16003, ADIS16060 and HMC6352.

\begin{tabular}{|c|c|}
\hline Parameter & Typical Value \\
\hline \multicolumn{2}{|c|}{ Gyroscope ADIS16060 } \\
\hline Measurement range & $\pm 80 \% / \mathrm{s}$ \\
\hline Sensitivity & $0.0122^{\circ} / \mathrm{s} / \mathrm{LSB}$ \\
\hline $\begin{array}{c}\text { Drift } \\
\end{array}$ & $0.1 \% / \mathrm{s}$ \\
\hline "Angular Rate Random Walk & $0.04^{\circ} / \mathrm{s} / \sqrt{\mathrm{Hz}}$ \\
\hline \multicolumn{2}{|c|}{ Accelerometer ADIS16003 } \\
\hline Measurement range & $\pm 1.7 \mathrm{~g}$ \\
\hline Sensitivity & $820 \mathrm{LSB} / \mathrm{g}$ \\
\hline Zero g Bias Stability & $\pm 8.5 \mathrm{mg}$ \\
\hline Acceleration Random Walk at Xout, Yout & $110 \mu \mathrm{g} / \sqrt{\mathrm{Hz}} \mathrm{RMS}$ \\
\hline \multicolumn{2}{|c|}{ "Magnetic Sensor HMC6352 } \\
\hline Heading Accuracy & 2.5 degRMS \\
\hline Heading Resolution & $0.5 \mathrm{deg}$ \\
\hline Disturing Field & min 20 gauss \\
\hline Max. Exposed Field & $\max 10,000$ gauss \\
\hline
\end{tabular}




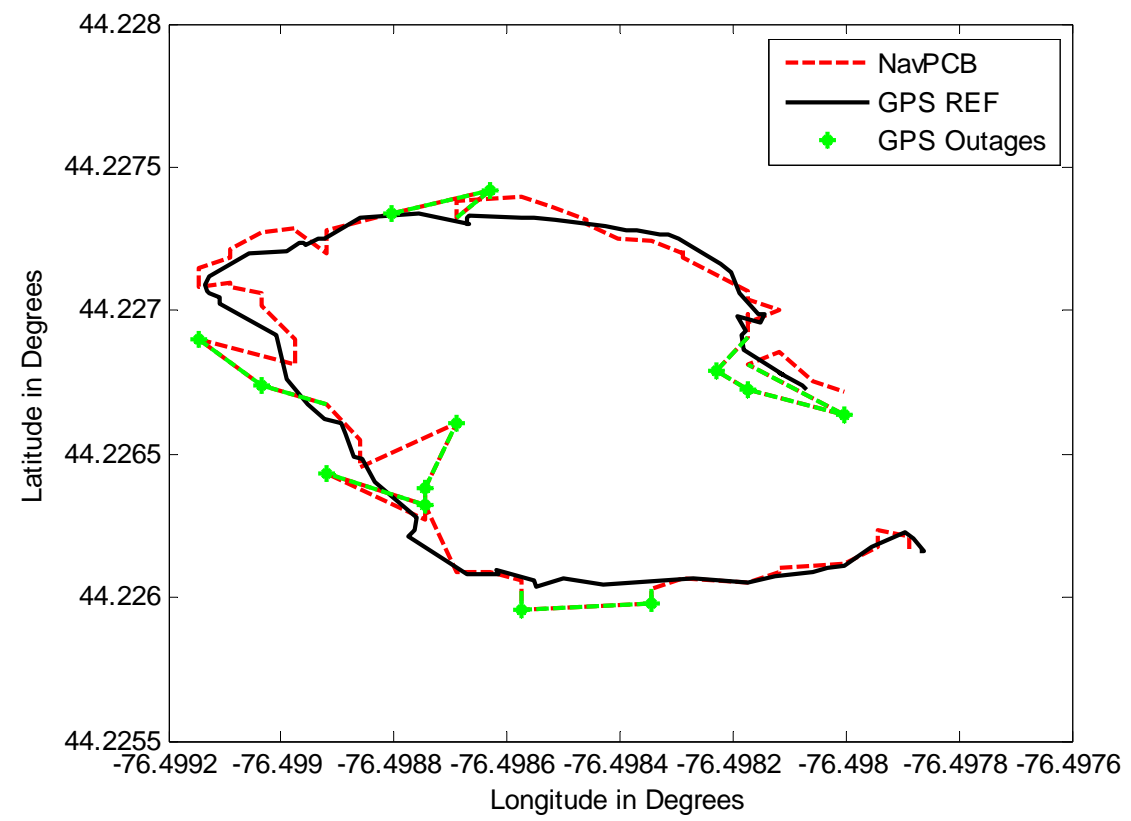

Figure 5.23 Test III.1: GPS trajectory and on-foot Trajectory with 6 GPS outages.

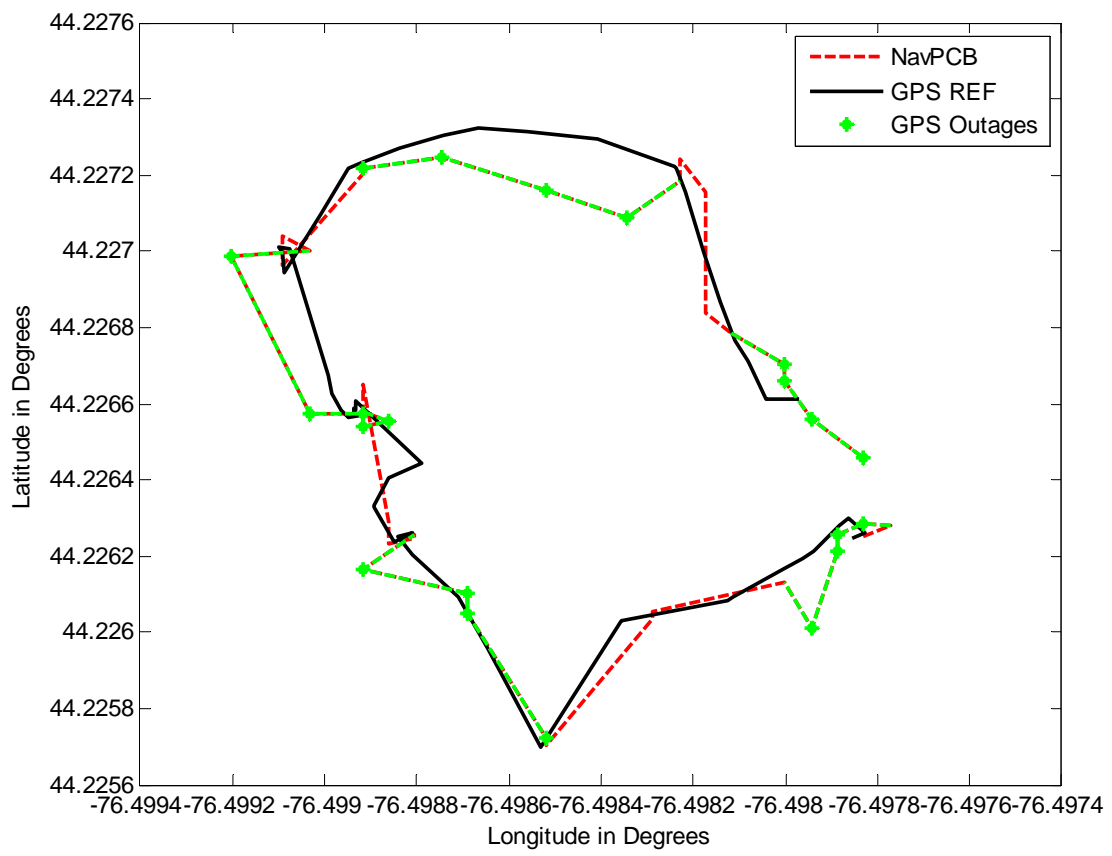

Figure 5.24 Test III.2: GPS trajectory and on-foot trajectory with 5 GPS outages. 


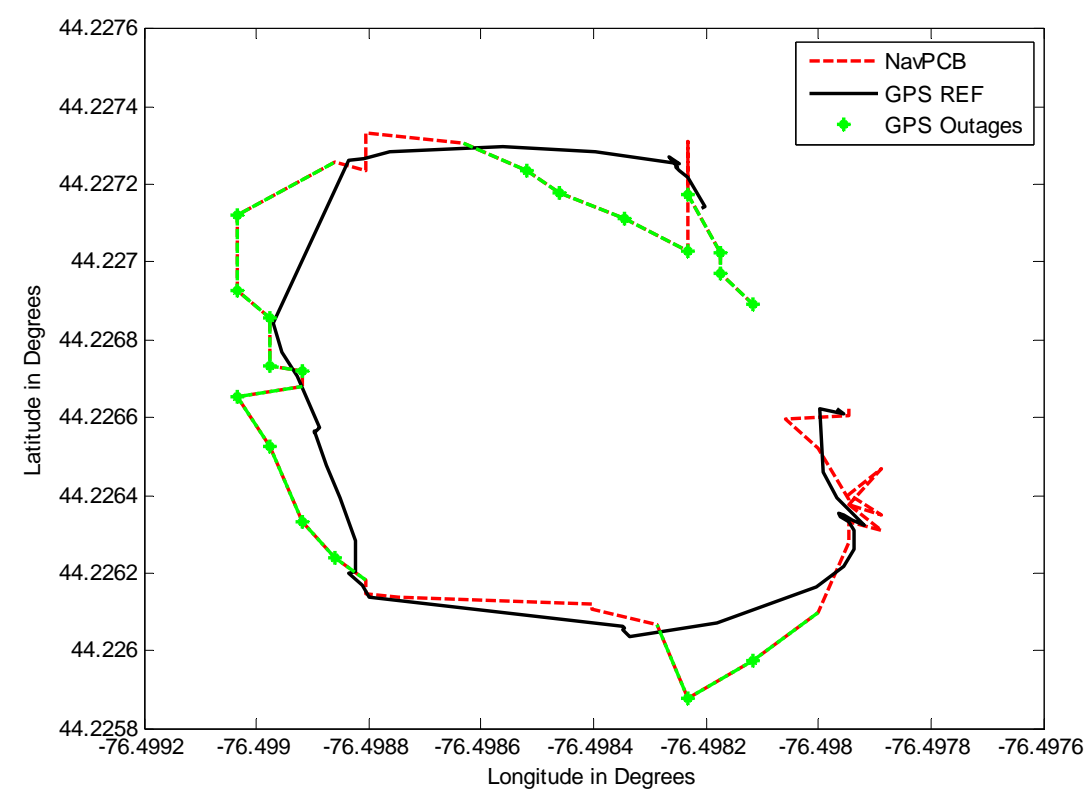

Figure 5.25 Test III.3: GPS trajectory and on-foot trajectory with 5 GPS outages.

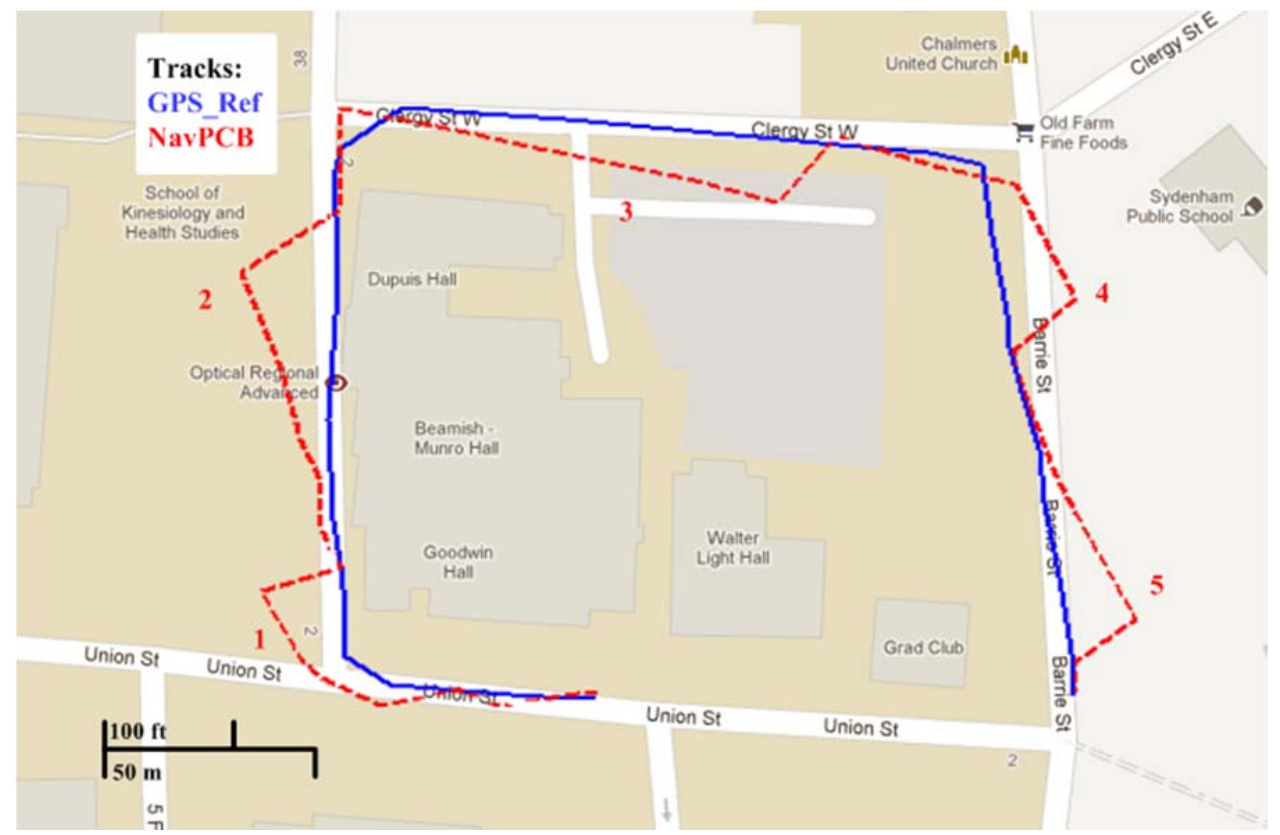

Figure 5.26 Test III.4: GPS trajectory and in-vehicle trajectory with 5 GPS outages. 


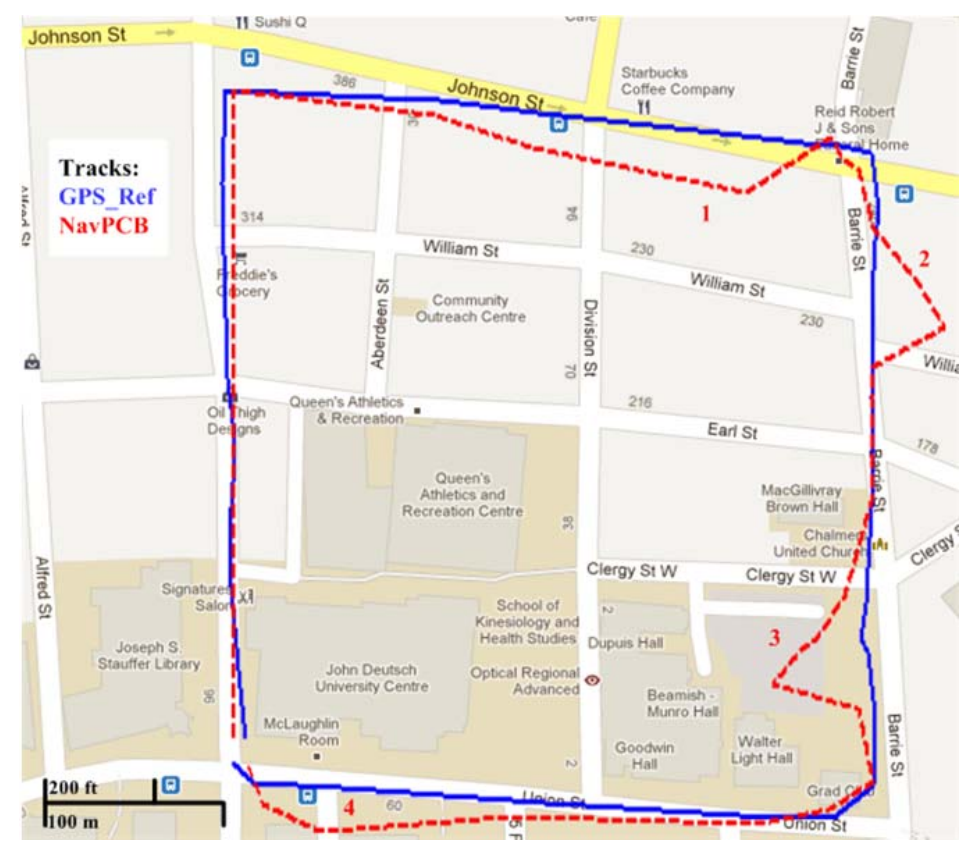

Figure 5.27 Test III.5: GPS trajectory and in-vehicle trajectory with 4 GPS outages.

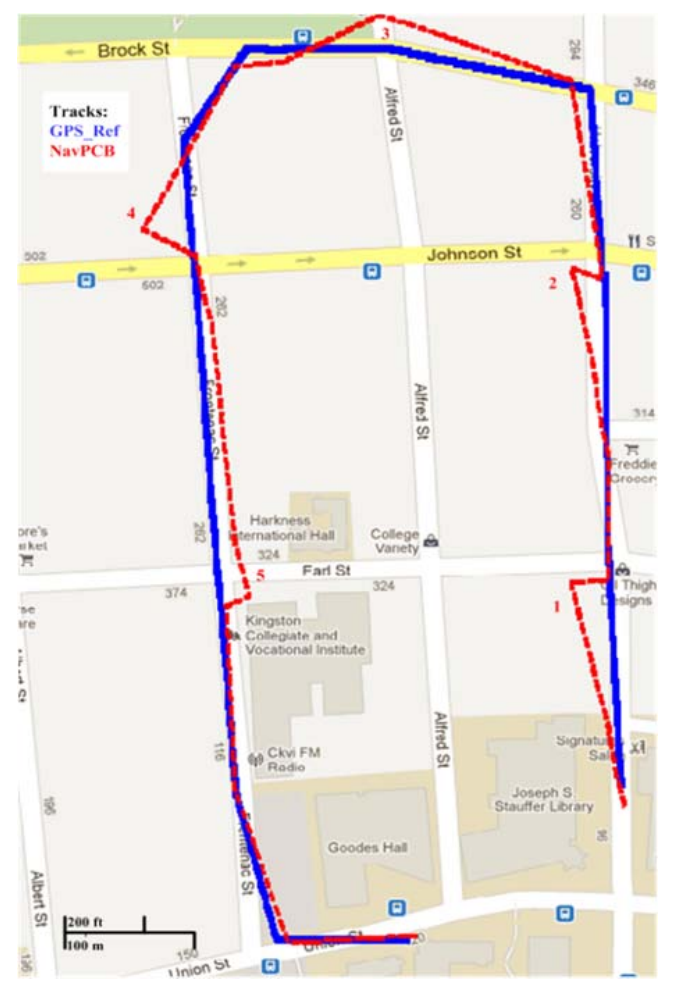

Figure 5.28 Test III.6: GPS trajectory and in-vehicle trajectory with 5 GPS outages. 
Table 5.12 On-foot field test: Position errors during GPS outages.

\begin{tabular}{|c|c|c|c|c|c|c|}
\hline \multicolumn{7}{|c|}{ Trajectory 1} \\
\hline & Outage 1 & Outage 2 & Outage 3 & Outage 4 & Outage 5 & Outage 6 \\
\hline $\begin{array}{l}\text { Outage Duration } \\
\text { in Seconds }\end{array}$ & 28 & 33 & 34 & 26 & 28 & 29 \\
\hline $\begin{array}{c}\text { Position Error } \\
\text { in Meters }\end{array}$ & 8.588 & 26.870 & 18.892 & 29.812 & 13.181 & 17.531 \\
\hline $\begin{array}{l}\text { Average Position } \\
\text { Error in Meters }\end{array}$ & \multicolumn{6}{|c|}{19.146} \\
\hline \multicolumn{7}{|c|}{ Trajectory 2} \\
\hline & Outage 1 & Outage 2 & Outage 3 & Outage 4 & Outage 5 & \\
\hline $\begin{array}{l}\text { Outage Duration } \\
\text { in Seconds }\end{array}$ & 32 & 34 & 27 & 31 & 33 & \\
\hline $\begin{array}{c}\text { Position Error } \\
\text { in Meters }\end{array}$ & 16.419 & 13.794 & 10.570 & 23.349 & 20.605 & \\
\hline $\begin{array}{c}\text { Average Position } \\
\text { Error } \\
\text { in Meters }\end{array}$ & \multicolumn{6}{|c|}{16.947} \\
\hline \multicolumn{7}{|c|}{ Trajectory 3} \\
\hline & Outage 1 & Outage 2 & Outage 3 & Outage 4 & Outage 5 & \\
\hline $\begin{array}{l}\text { Outage Duration } \\
\text { in Seconds }\end{array}$ & 34 & 31 & 31 & 35 & 31 & \\
\hline $\begin{array}{c}\text { Position Error } \\
\text { in Meters }\end{array}$ & 19.524 & 23.366 & 31.370 & 26.847 & 28.116 & \\
\hline $\begin{array}{c}\text { Average Position } \\
\text { Error } \\
\text { in Meters }\end{array}$ & \multicolumn{6}{|c|}{25.845} \\
\hline
\end{tabular}


Table 5.13 In-vehicle field test: Position errors during GPS outages.

\begin{tabular}{|c|c|c|c|c|c|}
\hline \multicolumn{6}{|c|}{ Trajectory 4} \\
\hline & Outage 1 & Outage 2 & Outage 3 & Outage 4 & Outage 5 \\
\hline $\begin{array}{l}\text { Outage Duration } \\
\text { in Seconds }\end{array}$ & 24 & 28 & 34 & 36 & 30 \\
\hline $\begin{array}{l}\text { Position Error } \\
\text { in Meters }\end{array}$ & 22.672 & 22.289 & 34.939 & 17.453 & 21.127 \\
\hline $\begin{array}{l}\text { Average Position } \\
\text { Error in Meters }\end{array}$ & \multicolumn{5}{|c|}{23.696} \\
\hline \multicolumn{6}{|c|}{ Trajectory 5} \\
\hline & Outage 1 & Outage 2 & Outage 3 & Outage 4 & \\
\hline $\begin{array}{l}\text { Outage Duration } \\
\text { in Seconds }\end{array}$ & 32 & 29 & 35 & 34 & \\
\hline $\begin{array}{c}\text { Position Error } \\
\text { in Meters }\end{array}$ & 30.900 & 25.692 & 33.308 & 26.803 & \\
\hline $\begin{array}{l}\text { Average Position } \\
\text { Error in Meters }\end{array}$ & \multicolumn{5}{|c|}{29.176} \\
\hline \multicolumn{6}{|c|}{ Trajectory 6} \\
\hline & Outage 1 & Outage 2 & Outage 3 & Outage 4 & Outage 5 \\
\hline $\begin{array}{l}\text { Outage Duration } \\
\text { in Seconds }\end{array}$ & 29 & 32 & 32 & 29 & 33 \\
\hline $\begin{array}{c}\text { Position Error } \\
\text { in Meters }\end{array}$ & 24.600 & 21.934 & 29.073 & 32.021 & 28.879 \\
\hline $\begin{array}{l}\text { Average Position } \\
\text { Error in Meters }\end{array}$ & \multicolumn{5}{|c|}{27.301} \\
\hline
\end{tabular}

\subsection{Summary}

The objective of the field tests was to evaluate the navigation performance of the integrated system using low-cost MEMS inertial sensors and the GPS. Open area tests are discussed in this chapter to investigate the system performance with different GPS signal visibilities. Many experiments were repeated and the overall system performance results were positive. In this chapter, the results of three field tests were presented. These results were compared based on INS-only navigation and therefore, several simulated GPS outage periods were intentionally inserted into the first and second tests. In the third test, the GPS outages were inserted by removing GPS directly from the navigation board. For 
the first test, wheel encoders and a high-performance MEMS gyroscope were used and the outage duration was 60 seconds. The maximum RMS value of the position errors among all these 4 outages is 8.9 meters. If the vehicle ran in a straight line, the maximum RMS value of the position errors was less than 5 meters. The second test used more compact and less expensive MEMS inertial sensors to provide the accelerations and the angular rate. A successful 2D KF algorithm was presented, as well as the constraints of the vehicle motion being employed in the second field test, which decreased the position/velocity errors to some extent. Due to the error propagation characteristics of MEMS sensors, the errors quickly grew when 20-second GPS signal gaps were inserted. However, control of the navigation errors was sufficient during short GPS outages. The maximum RMS value of the position errors was less than 11.5 meters. The results also show that the navigation errors are reduced by NHC during GPS outages, with an average improvement level of approximately 1.4 meters. The third test evaluated the real-time performance of the navigation board. The results from the trajectories show that by using the navigation board, the average position errors during roughly 30 -second GPS outages are approximately 20.6 meters for the on-foot scenario and 26.7 meters for the in-vehicle scenario. 


\section{Chapter 6}

\section{Summary, Conclusions and Recommendations}

\subsection{Summary of Conclusions}

The main contribution of this thesis is to propose an integrated system architecture for GPS/INSenable WSN. This hardware architecture is a recommended implementation method for locationbased WSN since it is highly optimized for dimension, real-time computation and energy efficiency. Besides, the INS/GPS integration makes the system capable to get reliable navigation information when GPS signals are weak or blocked. The proposed system uses a single DSP chip for both the data acquisition and inertial navigation computations. A fifteen state Kalman filter was designed using the perturbation theory model for position, velocity and attitude. Field tests were also carried out to assess the real-time performance and the overall system accuracy. GPS outages were simulated and the INS prediction accuracy was evaluated for the field data.

The issues regarding GPS/INS integration, software/hardware implementation, real-time performance and field test results are summarized below:

1. The proposed system is optimized for dimension, real-time computation and power consumption, which are the focal concerns in energy-constrained sensor networks. A loosely coupled GPS/INS integrated approach is applied to estimate the position, velocity and attitude. This navigation board uses a fixed-point DSP with a clock rate of $100 \mathrm{MHz}$ to perform the navigation calculation and one single power supply of $4.5 \mathrm{~V}$ is used to feed all the circuit boards. MEMS sensors are used in this proposed system to reduce the overall cost, weight and power consumption. 
2. When the system is employed in an urban canyon or forest, where GPS becomes unavailable due to signal blockages, the integration accuracy is degraded. In the first scenario in Chapter 5, the vehicle used wheel encoders and a high-performance MEMS gyroscope to obtain the acceleration and angular rate. Based on the trial results, the average position error of this proposed system was 5 meters during a 60-second GPS outage. The largest RMS value of the position error among the four outages was less than 9 meters. In the second test, when less expensive inertial sensors were used and the average speed of the vehicle was approximately 1.45 meters/second, the position error was 10.17 meters on average during a 20 -second GPS outage. The largest RMS value of position errors among the four outages was less than 14.5 meters.

3. Low-power design: a PIC microcontroller is used to detect the external-interrupt triggered motion/acceleration of the navigation board and control the power supplies of various circuits and ICs. The motion detection scheme takes advantage of the ultra-low-power wake-up feature of MCU PIC16F886, which significantly decreases the power consumption of the board when it remains stationary.

4. Real-time performance: The DSP works on a $100 \mathrm{MHz}$ clock rate and the Kalman filter was executed every $47.6 \mathrm{~ms}$. This is well within the real-time requirements and achieves positioning accuracy. The GPS updates once per second whenever it is available.

5. An RF transceiver is integrated into the navigation board. Therefore, this system can be used in Zigbee sensor networks. Each moving end device can send its positioning data to the cluster header/network coordinator over the air. 
6. The stand-alone INS position errors can be decreased by the use of non-holonomic constraints. Based on the test results in Chapter 5, the non-holonomic constraints provided a better navigation solution and it achieved an average improvement of approximately 1.4 meters during the GPS outages.

7. The real-time tests by using the proposed navigation board with the improvement provided by a magnetic sensor have been carry out to evaluate the system performance with actual sensor inputs. The results show that the average position error during a 30-second GPS outage is approximately 20.6 meters for the on-foot scenario and 26.7 meters for the in-vehicle scenario.

\subsection{Developed Programs}

The following is a list of programs developed for implementing and testing the embedded system:

1. A motion detection program based on MPLAB IDE that can detect the motion of the board and manage the system power (Chapter 4)

2. A navigation system program developed in CCS that includes DSP initialization and configurations, ISRs, data acquisition, data parsing, navigation computation algorithms, communications and interfaces with the PIC MCU and CC2430 (Chapter 4)

3. Programs developed in IAR Embedded Workbench that describe the application layer of end devices, routers and the coordinator based on ZigBee sensor networks, as well as interface programs for the CC2430 MCU to communicate with the external flash memory and DSP (Chapter 4) 
4. A Matlab-based loosely coupled EKF that is used to simulate and analyze the system performance of the GPS/INS integrated solution (Chapter 5)

5. A program that logs and analyzes the static data from inertial sensors to estimate the deterministic error parameters and the random error parameters for $1^{\text {st }}$ order GM model (Chapter 5)

\subsection{Recommendations for Future Work}

Based on the results presented in the previous chapters, the following recommendations for future work to improve the performance of the proposed navigation system are made and listed as follows:

1. As discussed in Chapter 3, initial calibration and stochastic error modeling are essential to improve the KF prediction accuracy during GPS outages. Although the $1^{\text {st }}$ order GM model reduces the computational complexity of the Kalman filter, using higher order GM models or AR models will provide better results, which can be compared with the traditional $1^{\text {st }}$ order GM model regarding the accuracy improvement.

2. Low cost MEMS inertial sensors have run-to-run biases. An efficient way to calibrate the sensor biases is to use on-line GPS data to calibrate the bias errors and reset them in real-time.

3. A 3D solution for the GPS/INS integration scheme with 6 degree-of-freedom inertial sensor array can be applied to the system and the navigation results will be compared with a $2 \mathrm{D}$ solution to determine whether and to what degree it can improve the integration accuracy during GPS outages. 
4. A possible extension of this work would be to investigate new methods to improve the accuracy of this navigation solution during GPS outages, without significantly increasing the computation complexity of the algorithm. 


\section{Bibliography}

1. Ko, Y.B. and N.H. Vaidya, Location-Aided Routing (LAR) in mobile ad hoc networks. Wireless Networks, 2000. 6(4): p. 307-321.

2. Zorzi, M. and R.R. Rao, Geographic random forwarding (GeRaF) for ad hoc and sensor networks: Multihop performance. Ieee Transactions on Mobile Computing, 2003. 2(4): p. 337-348.

3. V.Hnatyshin, H.A., Design and Implementation of an OPNET model for simulating GeoAODV MANET routing Protocol, in Proc. of the OPNETWORK 2010 International Conference, Session: Wireless Ad Hoc and Wireless Personal Area Networks. August, 2010: Washington DC.

4. Dipankar Deb, S.B.R., NabenduChaki, LACBER: A New Location Aided Routing Protocol for GPS Scarce MANET. International Journal of Wireless \& Mobile Networks (IJWMN), August 2009. 1.

5. Zhang, G., A Low Cost Integrated INS/GPS System. UCGE Reports Number 20078, 1995.

6. Elkaim, G.H., M. Lizarraga, and L. Pederseny, Comparison of low-cost GPS/INS sensors for Autonomous Vehicle applications. Position, Location and Navigation Symposium, 2008 IEEE/ION 2008: p. 1133-1144.

7. Kelley, R.T., I.N. Katz, and C.A. Bedoya, Design, development and evaluation of an Ada coded INS/GPS open loop Kalman filter, in Proceedings of the IEEE 1990 National, Aerospace and Electronics Conference. 1990. p. 382-388.

8. Chatfield, A., Fundamentals of High Accuracy Inertial Navigation. 1997: American Institute of Aeronautics and Astronautics.

9. Rogers, R.M., Applied mathematics in integrated navigation systems. 3rd ed. AIAA education series. 2007, Reston, VA: American Institute of Aeronautics and Astronautics. xvii, $408 \mathrm{p}$.

10. Noureldin, A., A. Osman, and N. El-Sheimy, A neuro-wavelet method for multi-sensor system integration for vehicular navigation. Measurement Science \& Technology, 2004. 15(2): p. 404-412.

11. Chiang, K.W., A. Noureldin, and N. El-Sheimy, A new weight updating method for INS/GPS integration architectures based on neural networks. Measurement Science \& Technology, 2004. 15(10): p. 2053-2061.

12. El-Sheimy, N., K.W. Chiang, and A. Noureldin, The utilization of artificial neural networks for multisensor system integration in navigation and positioning instruments. Ieee Transactions on Instrumentation and Measurement, 2006. 55(5): p. 1606-1615.

13. Shang, J., G. Mao, and Q.T. Gu, Design and implementation of MIMU/GPS integrated navigation systems. 2002 Ieee Position Location and Navigation Symposium, 2002: p. 99-105 366.

14. Qu, P.P., L. Fu, and X. Zhao, Design of Inertial Navigation System Based on Micromechanical Gyroscope and Accelerometer. Ccdc 2009: 21st Chinese Control and Decision Conference, Vols 1-6, Proceedings, 2009: p. 1351-1354 
6189.

15. Agarwal, V., H. Arya, and S. Bhaktavatsala, Design and Development of a Real-Time DSP and FPGA-Based Integrated GPS-INS System for Compact and Low Power Applications. Ieee Transactions on Aerospace and Electronic Systems, 2009. 45(2): p. 443-454.

16. ADS8364, Texas Instruments, datasheet. Available from: http://focus.ti.com/lit/ds/symlink/ads8364.pdf.

17. Titterton, D.H., et al., Strapdown inertial navigation technology. 2nd ed. IEE radar, sonar, navigation, and avionics series 17. 2004, Stevenage: Institution of Electrical Engineers. xvii, $558 \mathrm{p}$.

18. Hamilton, W.R., Lectures on quaternions; containing a systematic statement of a new mathematical method; of which the principles were communicated in 1843 to the Royal Irish academy; and which has since formed the subject of successive courses of lectures, delivered in 1848 and subsequent years, in the halls of Trinity college, Dublin: with numerous illustrative diagrams, and with some geometrical and physical applications. 1853, Dublin,: Hodges and Smith; etc. 3 p.1., 1xxii, 736 p., 11.

19. Salychev, O.S., Inertial Systems in Navigation and Geophysics. 1998: Bauman MSTU Press.

20. Noureldin, A., et al., Performance Enhancement of MEMS-Based INS/GPS Integration for Low-Cost Navigation Applications. IEEE Transactions on Vehicular Technology, 2009. 58(3): p. 1077-1096.

21. Petovello, M.G., Real-Time Integration of a Tactical-Grade IMU and GPS for HighAccuracy Positioning and Navigation, in The Department of Geomatics Engineering. 2003, University of Calgary: Calgary. p. UCGE Preort \#20173.

22. Lijun, W., Y. Xiaoniu, and Z. Huichang, The Modeling and Analysis for Autonomous Navigation System Based on Tightly Coupled GPS/INS, in International Conference on Microwave and Millimeter Wave Technology. 2007. p. 1-4.

23. Miller, I., et al., Tightly-coupled GPS / INS system design for autonomous urban navigation. Position, Location and Navigation Symposium, 2008 IEEE/ION, 2008: p. 1297-1310.

24. Syed, Z., et al., Improved Vehicle Navigation Using Aiding with Tightly Coupled Integration, in IEEE Vehicular Technology Conference. 2008. p. 3077-3081.

25. IEEE Standard for Inertial Sensor Terminology. 2001: IEEE Std 528-2001.

26. El-Sheimy, N., Inertial techniques and INS/DGPS Integration, in Department of Geomatics Engineering. 2007, University of Calgary: Calgary.

27. Aggarwal, P., et al., A standard testing and calibration procedure for low cost MEMS inertial sensors and units. Journal of Navigation, 2008. 61(2): p. 323-336.

28. Godha, S., Performance Evaluation of Low Cost MEMS-Based IMU Integrated With GPS for Land Vehicle Navigation Application, in Department of Geomatics Engineering. 2006, University of Calgary: Calgary. p. 12.

29. Abdel-Hamid, W., A. Osman, A. Noureldin and N. El-Sheimy. Improving the Performance of MEMS-based Inertial Sensors by Removing Short-Term Errors Utilizing Wavelet MultiResolution Analysis. in Proceedings of the US Institute of Navigation (ION), National Technical Meeting (NTM). 2004. San Diego, CA. 
30. Chiang, K.W., Goodwill, C. and El-Sheimy, N., Improving the accuracy of INS/DGPS Integrated Navigation Systems, a Cascade Denoising approach. European Journal of Navigation 2007.

31. Papoulis, A. and S.U. Pillai, Probability, random variables, and stochastic processes. 4th ed. 2002, Boston: McGraw-Hill. x, 852 p.

32. Sukkarieh, S., Low Cost, High Integrity, Aided Inertial Navigation Systems for Autonomous Land Vehicles, in Australian Centre for Field Robotics, Dept. of Mechanical and Mechatronic Engineering. 2000, University of Sydney: Sydney, Australia.

33. Shin, E.-H., Accuracy Improvement of Low Cost INS/GPS for Land Applications, in Department of Geomatics Engineering. 2001, University of Calgary: Calgary, Canada. p. 7880.

34. ADIS16060, Analog Devices Inc., datasheet. Available from: http://www.analog.com/static/imported-files/data_sheets/ADIS16060.pdf.

35. ADIS16003, Analog Devices Inc., datasheet. Available from: http://www.analog.com/static/imported-files/data_sheets/ADIS16003.pdf.

36. LEA 5H, u-blox, datasheet. Available from: http://www.ublox.com/images/downloads/Product_Docs/LEA-5x_DataSheet_\%28GPS.G5-MS507026\%29.pdf.

37. TMS320VC5505, Texas Instruments, datasheet. Available from: http://focus.ti.com/lit/ds/symlink/tms320vc5505.pdf.

38. CC2430, Texas Instruments, datasheet. Available from: $\underline{\text { http://focus.ti.com/general/docs/lit/getliterature.tsp?genericPartNumber }=c c 2430 \& \text { fileType }=p}$ df.

39. TPS61032, Texas Instruments, datasheet.

40. TPS76601, Texas Instruments, datasheet. Available from: http://focus.ti.com/lit/ds/symlink/tps76601.pdf.

41. TPS3106K33, Texas Instruments, datasheet. Available from: http://focus.ti.com/lit/ds/symlink/tps3106k33.pdf.

42. Z-Stack Developer's Guide, Texas Instruments, Document Number: F8W-2006-0022.

43. CC2430DK Development Kit User Manual, Texas Instruments. Available from: http://focus.ti.com/lit/ug/swru133/swru133.pdf.

44. ADXL335, Analog Devices Inc., datasheet. Available from: http://www.analog.com/static/imported-files/data sheets/ADXL335.pdf.

45. LISY300AL, STMicroelectronics, datasheet. Available from: http://www.sparkfun.com/datasheets/Sensors/LISY300AL.pdf.

46. AD7856, Analog Devices Inc., datasheet. Available from: http://www.analog.com/static/imported-files/data_sheets/AD7856.pdf.

47. Noureldin, A., Mobile Multi-Sensor Systems. EE523, Royal Military College of Canada, Kingston, ON, 2008. 


\section{Appendix A}

\section{Schematics of the Navigation Board}

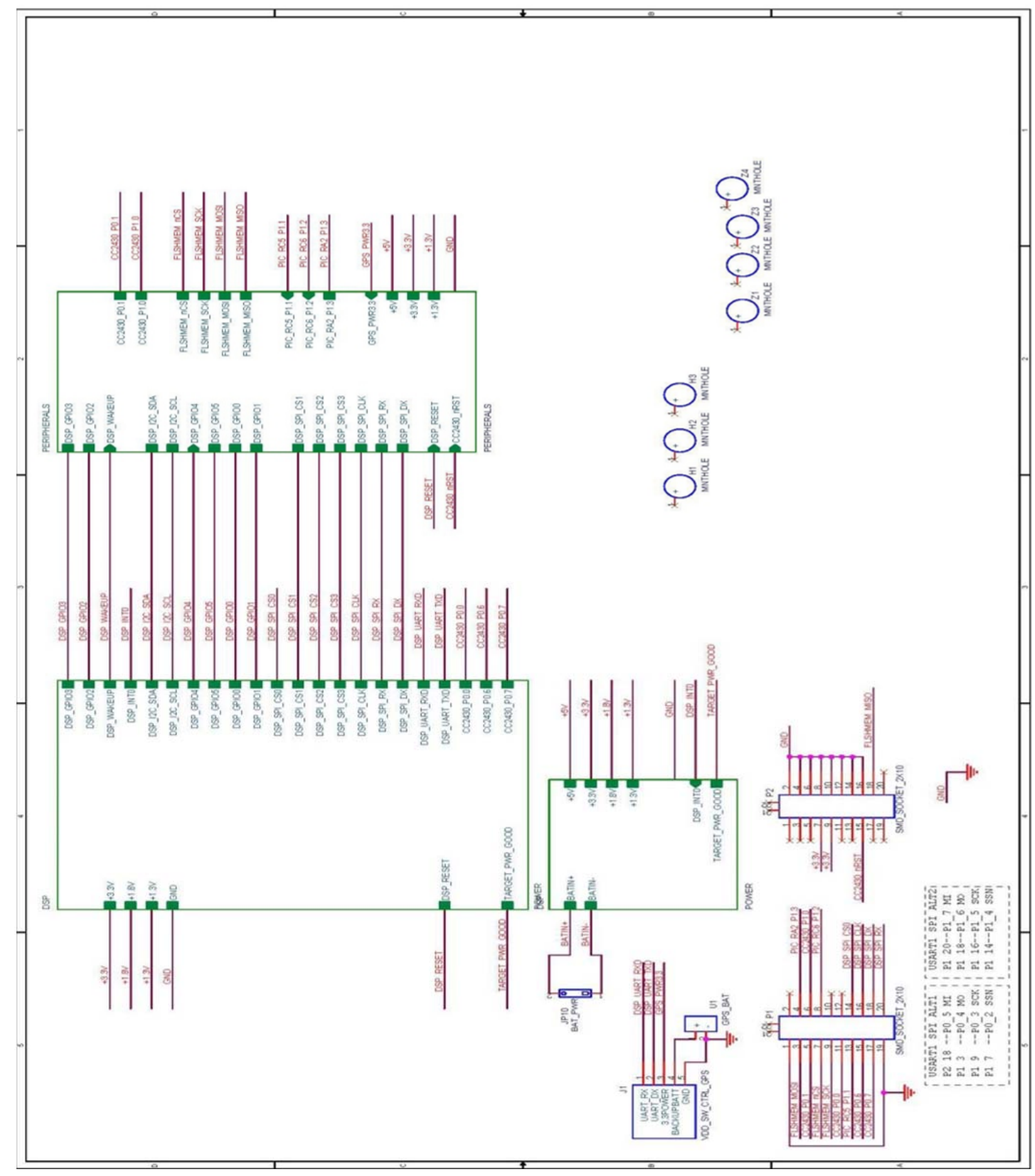

Figure A.1 Hierarchical block diagram of the navigation board. 


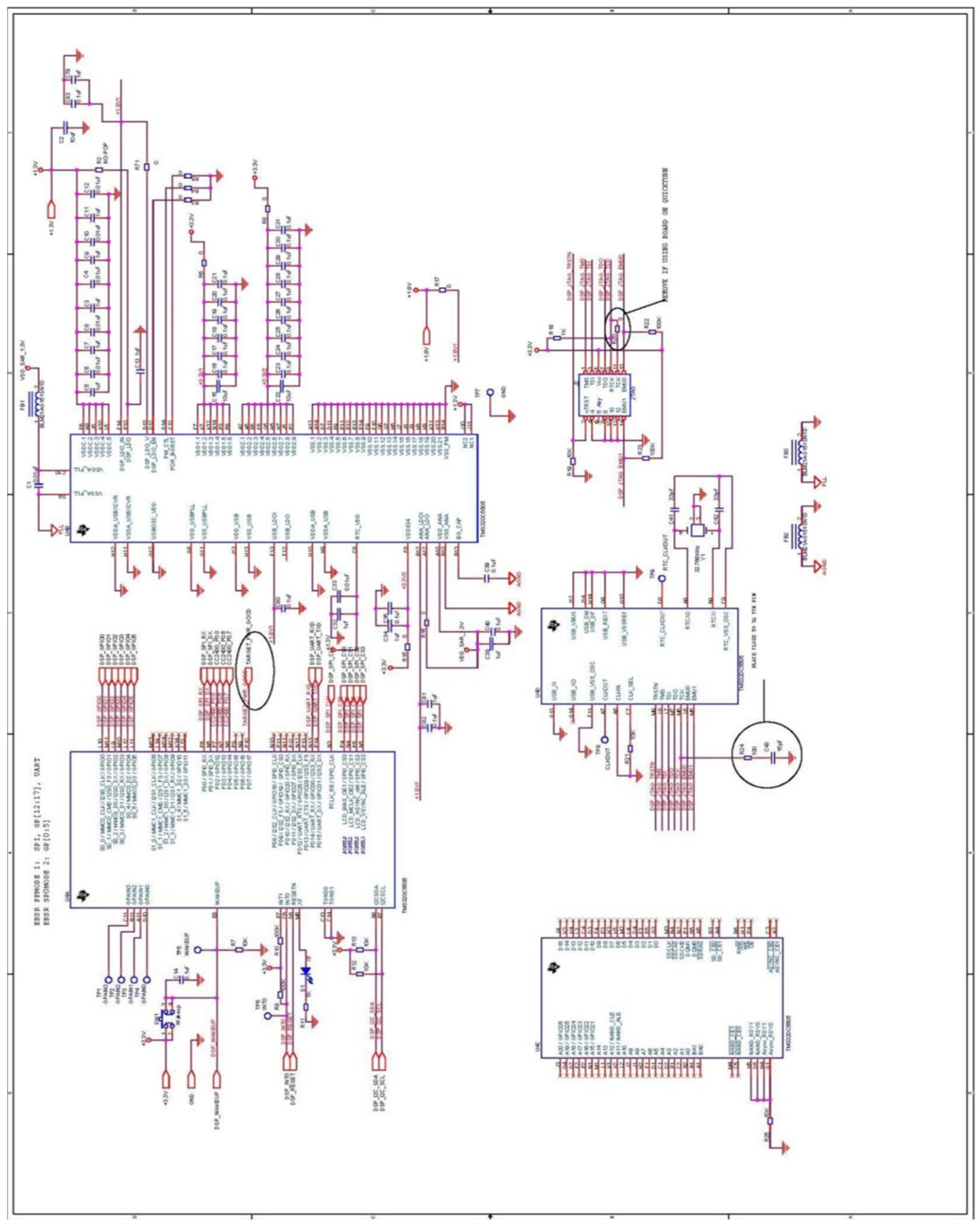

Figure A.2 Schematic of DSP TMS320VC5505. 


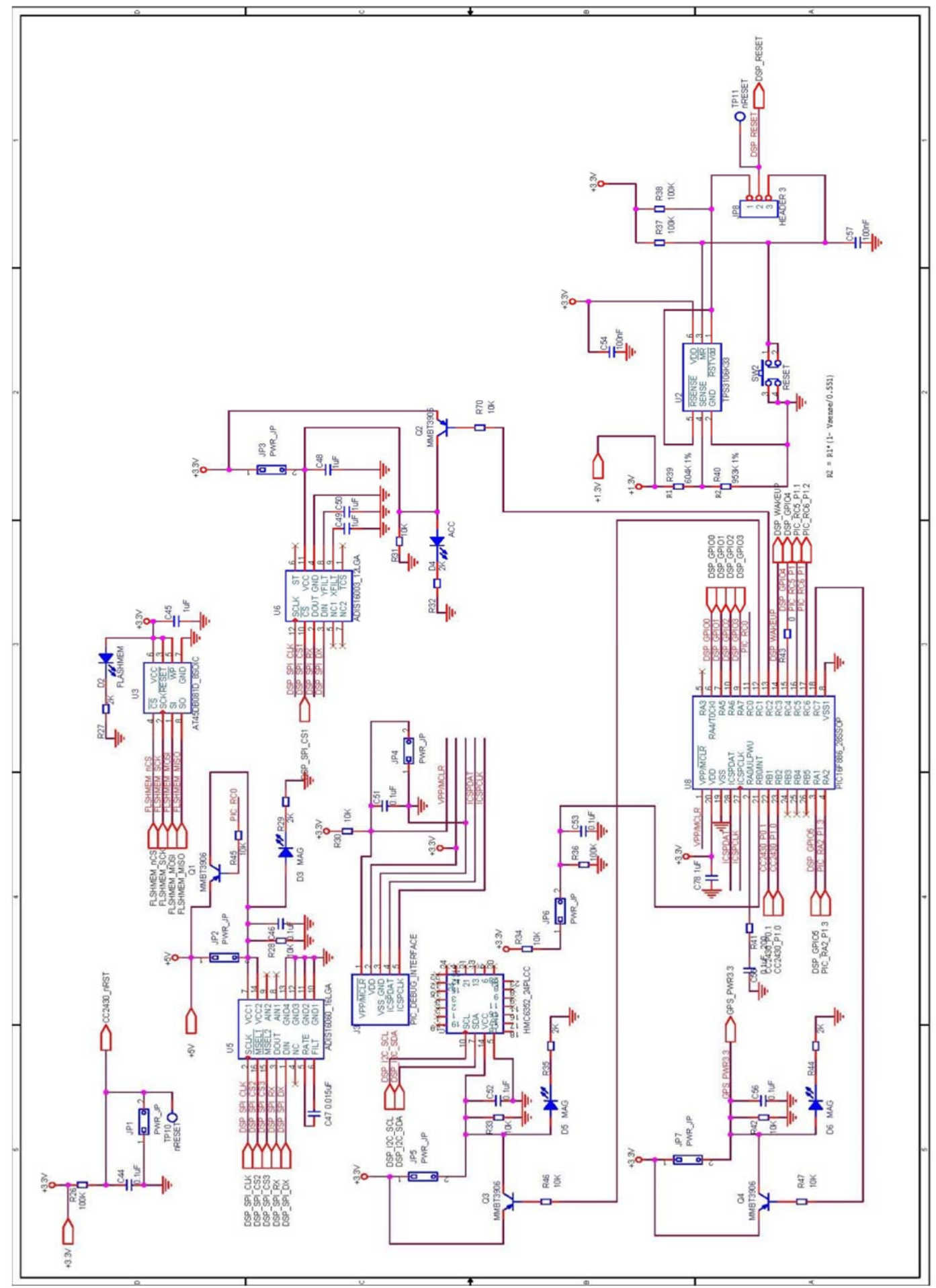

Figure A.3 Schematic of peripherals. 


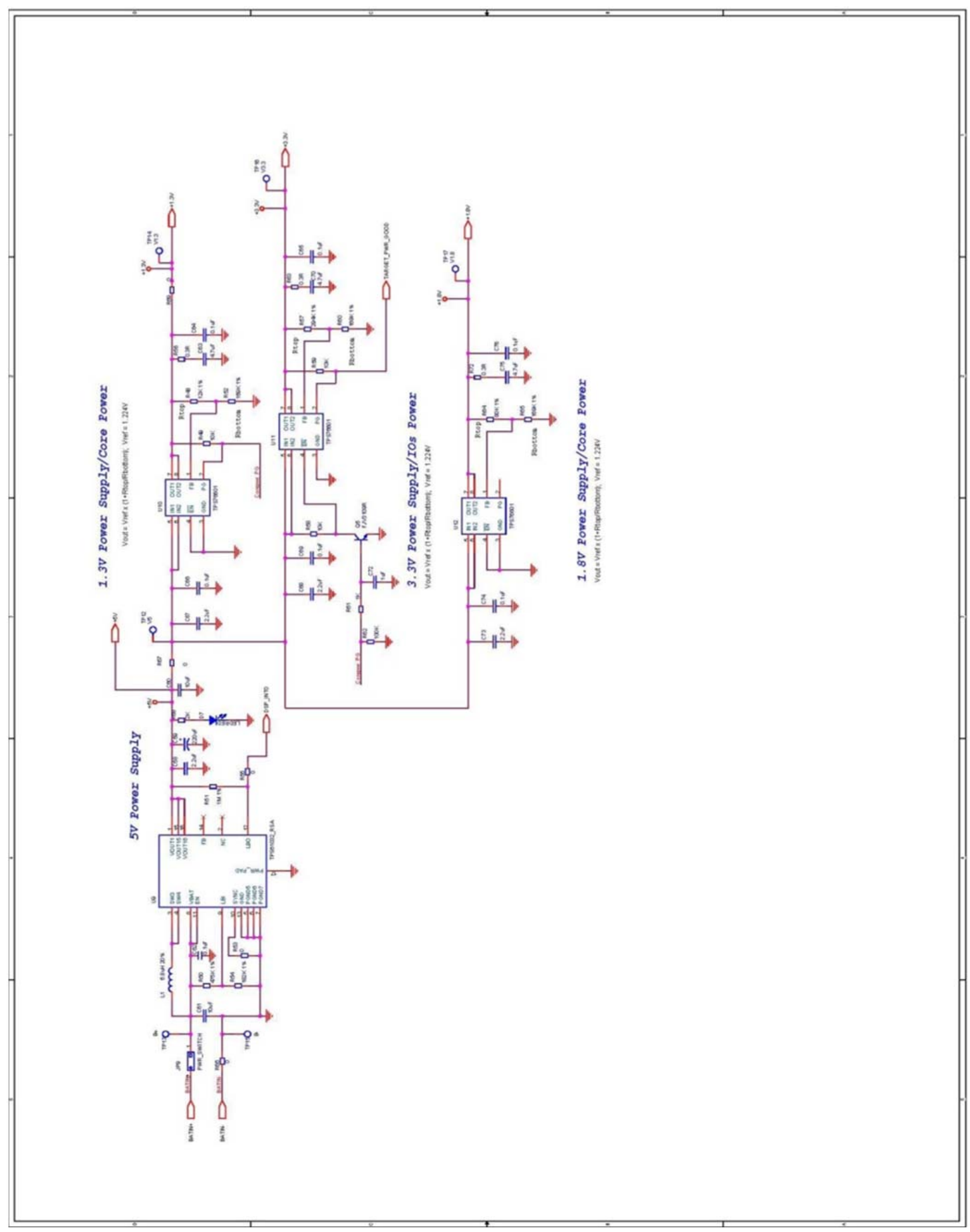

Figure A.4 Schematic of power supply. 